\title{
BUCKLING BEHAVIOR OF LONG ANISOTROPIC PLATES SUBJECTED TO ELASTICALLY RESTRAINED THERMAL EXPANSION
}

\author{
Michael P. Nemeth* \\ Mechanics and Durability Branch, NASA Langley Research Center \\ Hampton, Virginia 23681-2199
}

\begin{abstract}
An approach for synthesizing buckling results for, and behavior of, thin balanced and unbalanced symmetric laminates that are subjected to uniform heating or cooling and elastically restrained against thermal expansion or contraction is presented. This approach uses a nondimensional analysis for infinitely long, flexurally anisotropic plates that are subjected to combined mechanical loads and is based on useful nondimensional parameters. In addition, stiffness-weighted laminate thermal-expansion parameters and compliance coefficients are derived that are used to determine critical temperatures in terms of physically intuitive mechanical-buckling coefficients. The effects of membrane orthotropy and membrane anisotropy are included in the general formulation. Many results are presented for some common laminates that are intended to facilitate a structural designer's transition to the use of generic buckling design curves. Several curves that illustrate the fundamental parameters used in the analysis are presented, for nine contemporary material systems, that provide physical insight into the buckling response in addition to providing useful design data. Examples are presented that demonstrate the use of generic design curves. The analysis approach and generic results indicate the effects and characteristics of elastically restrained laminate thermal expansion or contraction, membrane orthotropy and anisotropy, and flexural orthotropy and anisotropy in a very general and unifying manner.
\end{abstract}

\section{Introduction}

Buckling behavior of laminated plates that are subjected to combined mechanical and thermal loads is an important consideration in the design of advanced highspeed aerospace vehicles. The sizing of many structural subcomponents of these vehicles is often determined by stability constraints. One element that is of practical importance in structural design is the long rectangular plate. These plates commonly appear as elements of stiffened panels that are used for wing structures, and as semimonocoque shell segments that are used for fuselage and launch vehicle structures. Buckling results for infinitely long

-Assistant Head. Associate Fellow. AIAA.

Copyright $\bigcirc 2002$ by the American Institute of Acronautics and Astronautics, Inc. No copyright is asserted in the United States under Title 17 . U. S. Corde. The A. S. Government has a royalty free ilcense to excreise all rights under the copyrigh plates are important because they often provide a practical estimate of the behavior of finite-length rectangular plates, and they provide information that is useful in explaining the behavior of these finite-length plates. Moreover, knowledge of the behavior of infinitely long plates can provide insight into the buckling behavior of more complex structures such as stiffened panels.

An important type of long plate that appears as an element of advanced composite structures is the symmetrically laminated plate. In the present study, the term, "symmetrically laminated," refers to plates in which every lamina, or ply, above the plate midplane has a corresponding lamina located at the same distance below the plate midplane, with the same thickness, material properties, and fiber orientation. Symmetrically laminated plates are essentially flat after the manufacturing process and exhibit flat prebuckling deformation states, which is desirable for many applications. More importantly, the amenability of these plates to structural tailoring provides symmetrically laminated plates with a significant potential for reducing the weight of aerospace vehicles or for meeting special performance requirements. Thus, understanding the mechanical and thermal buckling behavior of symmetrically laminated plates is an important part of the search for ways to exploit plate orthotropy and anisotropy to reduce structural weight or to fulfill a special design requirement.

In many practical cases, symmetrically laminated plates exhibit specially orthotropic behavior. However, in some cases, such as thin-walled $[ \pm 45]_{\mathrm{s}}$ laminates that are candidates for spacecraft applications, these plates exhibit anisotropy in the form of material-induced coupling between pure bending and twisting deformations. This coupling is referred to herein as flexural anisotropy and it generally yields buckling modes that are skewed in appearance (see Fig. 1). Unbalanced, symmetrically laminated plates are also being investigated for specialpurpose uses in aerospace structures. These laminated plates exhibit anisotropy in the form of material-induced coupling between pure inplane dilatation and inplane shear deformations in addition to flexural anisotropy. This coupling is referred to herein as membrane anisotropy and it generally yields combined inplane stress states for simple loadings like uniform edge compression when inplane displacement constraints are imposed on one or more edges of a plate. For example, when the edg- 
es of an unbalanced, symmetrically laminated plate, such as a $\left[+45_{2} / 0 / 90\right]_{s}$ laminate, are totally restrained against thermal expansion and contraction, that is caused by uniform heating or cooling, inplane shear stresses are developed in addition to the usual compressive stresses that are often present in balanced laminates. These kinematically induced shear stresses can be relatively large in magnitude, compared to the direct compressive stresses, and as a result can affect greatly the buckling behavior of the plate and yield buckling modes that are skewed in appearance.

The effects of flexural orthotropy and flexural anisotropy on the buckling behavior of long rectangular plates that are subjected to single and combined loading conditions are becoming better understood. For example, in-depth parametric studies that show the effects of flexural orthotropy and flexural anisotropy on the buckling behavior of long plates that are subjected to compression, shear, pure inplane bending, and various combinations of these loads have been presented in Refs. 1 through 3 . The results presented in these references indicate that the importance of flexural anisotropy on the buckling resistance of long plates varies with the magnitude and type of the combined loading condition. Similar results for plates loaded by uniform shear and a general linear distribution of axial load across the plate width have also been presented in Ref. 4 . In a similar manner, the effects of membrane orthotropy and membrane anisotropy on the buckling behavior of long rectangular plates that are restrained against axial thermal expansion or contraction and subjected to uniform heating or cooling and mechanical loads have been presented in Refs. 5 and 6. Likewise, similar results for plates that are fully restrained against thermal expansion and contraction and subjected to uniform heating or cooling have been presented in Ref. 7 . This work has provided a better understanding of the load interaction effects of balanced and unbalanced, symmetrically laminated plates that are subjected to mechanical loads and restrained against thermal expansion or contraction.

The effects of membrane orthotropy and anisotropy, and the effects of flexural orthotropy and anisotropy, on the buckling behavior of long rectangular plates that are restrained against thermal expansion and contraction and subjected to uniform heating or cooling are becoming better understood. However, relatively little work has been done that addresses, in a broad way, the effects of a compliant, elastic restraining medium on the thermal buckling of plates that are restrained against expansion and contraction. This problem is important because, in reality, it is impossible to completely restrain a plate from thermal expansion and contraction, and in many instances the restraining medium undergoes thermal deformations. Thus, one objective of the present study is to present an analytical approach that complements the physically intuitive buckling analysis presented in Ref. 7 for anisotropic plates that are fully restrained against thermal expansion or contraction and subjected to uniform heating or cooling. Another objective is to indicate the effects of a compliant, elastic restraining medium in a very general manner. Towards these objectives, an intuitive buckling analysis for symmetrically laminated plates, that are elastically restrained against thermal expansion and subjected to uniform heating or cooling, is presented that follows the analysis approach presented in Ref. 7, but includes the effects of the restraining medium in a very general manner. To achieve this objective, the buckling analysis is formulated directly in terms of buckling coefficients for the known, mechanically equivalent loads, stiffness-weighted laminate thermal expansion parameters, and compliance coefficients for the restraining medium. Emphasis is placed on the properties of the compliance coefficients for the restraining medium because once known, they can be used with the generic results presented in Ref. 7 for anisotropic plates that are fully restrained against thermal expansion and contraction to determine the buckling behavior for a very broad range of laminate constructions and restraining media. Thus, another objective of the proposed paper is to present values of the compliance coefficients for the restraining medium for a wide range of laminate stacking sequences and material systems.

To accomplish the objectives of the present study, the analysis that is used to formulate thermal buckling problem in a generic nondimensional form is described first. This description includes the nondimensional buckling analysis for infinitely long symmetrically laminated plates that are subjected to combined mechanical loads, and the formulation for the thermally induced mechanical loads and critical temperature change. Then, examples of compliance coefficients for a laboratorytype elastic restraining medium are presented. Next, specific results are presented for several common laminates with the two long edges clamped or simply supported and all edges elastically restrained against inplane movement. Generic results are then presented that illustrate the fundamental parameters that are used in the analysis for nine contemporary material systems. Lastly, examples are presented that use the generic buckling-design curves presented herein and in Ref. 7, that are applicable to a very wide range of laminate constructions, to demonstrate the analytical procedure and illustrate the effects of a restraining medium on the buckling behavior.

\section{Analysis Description}

In preparing generic design charts for buckling of a single flat thin plate, a special-purpose analysis is often 
preferred over a general-purpose analysis code, such as a finite-element code, because of the cost and effort that is usually involved in generating a large number of results with a general-purpose code. The results presented in the present study were obtained by using such a special-purpose buckling analysis that is based on classical laminated-plate theory. The analysis details are lengthy; hence, only a brief description of the buckling analysis is presented herein. First, the buckling analysis for long plates that are subjected to a general set of mechanical loads is described. Then, the mechanical loads that are induced by elastically restrained thermal expansion and contraction and that are used in the buckling analysis are derived, and an expression for the critical temperature change is presented that is in terms of the corresponding crtitical loading parameter and mechanical-buckling coefficients.

\section{Buckling Analysis}

Symmetrically laminated plates can have many different constructions because of the wide variety of material systems, fiber orientations, and stacking sequences that can be selected to construct a laminate. A way of coping with the large number of choices for laminate constructions is to use convenient nondimensional parameters in order to understand overall behavioral trends and sensitivities of the structural behavior to changes in laminate construction. The buckling analysis used in the present paper is based on classical laminated-plate theory and the classical Rayleigh-Ritz method, and is derived explicitly in terms of the nondimensional parameters defined in Refs. 1-8. This approach was motivated by the need for generic (independent of a specific laminate construction) parametric results for composite-plate buckling behavior that are expressed in terms of the minimum number of independent parameters needed to fully characterize the behavior, and that indicate the overall trends and sensitivity of the results to changes in the parameters. The nondimensional parameters that were used to formulate the buckling analysis are given by

$$
\begin{aligned}
& \alpha_{\infty}=\frac{b}{\lambda}\left(\frac{D_{11}}{D_{22}}\right)^{1 / 4} \\
& \beta=\frac{D_{12}+2 D_{6 \delta}}{\left(D_{11} D_{22}\right)^{1 / 2}} \\
& \gamma=\frac{D_{16}}{\left(D_{11}^{3} D_{22}\right)^{1 / 4}} \\
& \delta=\frac{D_{26}}{\left(D_{11} D_{22}^{3}\right)^{1 / 4}}
\end{aligned}
$$

where $b$ is the plate width and $\lambda$ is the half-wave length of the buckle pattern of an infinitely long plate (see Fig. 1). The subscripted D-terms are the bending stiffnesses of classical laminated-plate theory. The parameters $\alpha_{\infty}$ and $\beta$ characterize the flexural orthotropy, and the parameters $\gamma$ and $\delta$ characterize the flexural anisotropy.

The mechanical loading conditions that are included in the buckling analysis are uniform transverse tension or compression, uniform shear, and a general linear distribution of axial load across the plate width, as depicted in Fig. 1. Typically, an axial stress resultant distribution is partitioned into a uniform part and a pure bending part. However, this representation is not unique. The longitudinal stress resultant $N_{x}$ is partitioned in the analysis into a uniform tension or compression part and a linearly varying part that corresponds to eccentric inplane bending loads. This partitioning is given by

$$
\mathrm{N}_{\mathrm{x}}=\mathrm{N}_{\mathrm{xc}}-\mathrm{N}_{\mathrm{h}}\left[\varepsilon_{0}+\left(\varepsilon_{1}-\varepsilon_{0}\right) \eta\right]
$$

where $N_{x c}$ denotes the intensity of the constant-valued tension or compression part of the load, and the term containing $N_{h}$ defines the intensity of the eccentric inplane bending load distribution. The symbols $\varepsilon_{0}$ and $\varepsilon_{1}$ define the distribution of the inplane bending load, and the symbol $\eta$ is the nondimensional coordinate given by $\eta=y / b$. This particular way of partitioning the longitudinal stress resultant was used for convenience by eliminating the need to calculate the uniform and pure bending parts of an axial stress resultant distribution prior to performing a buckling analysis.

The analysis is based on a general formulation that includes combined destabilizing loads that are proportional to a positive-valued loading parameter $\tilde{\mathrm{p}}$ that is increased until buckling occurs, and independent subcritical combined loads that remain fixed at a specified load level below the value of the buckling load. Herein, the term "subcritical load" is defined as any load that does not cause buckling to occur. In practice, the subcritical loads are applied to a plate prior to, and independent of, the destabilizing loads with an intensity below that which will cause the plate to buckle. Then, with the subcritical loads fixed, the active, destabilizing loads are applied by increasing the magnitude of the loading parameter until buckling occurs. This approach permits certain types of combined-load interaction to be investigated in a direct and convenient manner. For example, in analyzing the stability of an aircraft fuselage, the nondestabilizing transverse tension load in a fuselage panel that is caused by cabin pressurization can be considered to remain constant and, as a result, it can be represented as a passive, subcritical load. The combined shear, compression, and inplane bending loads that are caused by flight maneuvers can vary and cause buckling and, as a result, they can be represented as active, destabilizing loads.

The distinction between the active, destabilizing 
and passive subcritical loading systems is implemented in the buckling analysis by partitioning the prebuckling stress resultants as follows

$$
\begin{aligned}
& N_{x c}=-N_{x 1}^{c}+N_{x 2}^{c} \\
& N_{y}=-N_{y 1}+N_{y 2} \\
& N_{x y}=N_{x y 1}+N_{x y 2} \\
& N_{b}=N_{h 1}+N_{b 2}
\end{aligned}
$$

where the stress resultants with the subscript 1 are the destabilizing loads, and those with the subscript 2 are the subcritical loads. The sign convention used herein for positive values of these stress resultants is shown in Fig. 1. In particular, positive values of the general linear edge stress distribution parameters $\mathrm{N}_{\mathrm{b} 1}, \mathrm{~N}_{\mathrm{b} 2}, \varepsilon_{0}$, and $\varepsilon_{1}$ correspond to compression loads. Negative values of $N_{h 1}$ and $N_{h 2}$, or negative values of either $\varepsilon_{0}$ or $\varepsilon_{1}$, yield linearly varying stress distributions that include tension. Depictions of a variety of inplane bending load distributions are given in Ref. 4. The two normal stress resultants of the system of destabilizing loads, $\mathrm{N}_{\mathrm{x} 1}^{\mathrm{c}}$ and $\mathrm{N}_{\mathrm{y}}$, are defined to be positive-valued for compression loads. This convention results in positive eigenvalues being used to indicate instability due to uniform compression loads.

The buckling analysis includes several nondimensional stress resultants associated with Eqs. (6) through (9). These dimensionless stress resultants are given by

$$
\begin{aligned}
& \mathrm{n}_{\mathrm{xj}}^{\mathrm{c}}=\frac{\mathrm{N}_{\mathrm{xj}}^{\mathrm{c}} \mathrm{b}^{2}}{\pi^{2}\left(\mathrm{D}_{11} \mathrm{D}_{22}\right)^{1 / 2}} \\
& \mathrm{n}_{\mathrm{yj}}=\frac{\mathrm{N}_{\mathrm{yj}} \mathrm{b}^{2}}{\pi^{2} D_{22}} \\
& \mathrm{n}_{\mathrm{xyj}}=\frac{\mathrm{N}_{\mathrm{xyj}} \mathrm{b}^{2}}{\pi^{2}\left(\mathrm{D}_{11} D_{22}^{3}\right)^{1 / 4}} \\
& \mathrm{n}_{\mathrm{bj}}=\frac{\mathrm{N}_{\mathrm{bj}} \mathrm{b}^{2}}{\pi^{2}\left(\mathrm{D}_{11} D_{22}\right)^{1 / 2}}
\end{aligned}
$$

where the subscript $\mathrm{j}$ takes on the values of 1 and 2 . In addition, the destabilizing loads are expressed in terms of the loading parameter $\tilde{p}$ in the analysis by

$$
\begin{aligned}
& \mathrm{n}_{\mathrm{x} 1}^{\mathrm{c}}=\mathrm{L}_{1} \tilde{\mathrm{p}} \\
& \mathrm{n}_{\mathrm{y} 1}=\mathrm{L}_{2} \widetilde{\mathrm{p}} \\
& \mathrm{n}_{\mathrm{xy} 1}=\mathrm{L}_{3} \tilde{\mathrm{p}} \\
& \mathrm{n}_{\mathrm{b} 1}=\mathrm{L}_{4} \widetilde{\mathrm{p}}
\end{aligned}
$$

where $\mathrm{L}_{1}$ through $\mathrm{L}_{4}$ are load factors that determine the specific form (relative contributions of the load components) of a given system of destabilizing loads. Typically, the dominant load factor is assigned a value of 1 and all others are given as positive or negative fractions.

Nondimensional buckling coefficients that are used herein are given by the values of the dimensionless stress resultants of the system of destabilizing loads at the onset of buckling; i.e.,

$$
\begin{aligned}
& \mathrm{K}_{\mathrm{x}} \equiv\left(\mathrm{n}_{\mathrm{x} 1}^{\mathrm{c}}\right)_{\mathrm{cr}}=\frac{\left(\mathrm{N}_{\mathrm{x} 1}^{\mathrm{c}}\right)_{\mathrm{cr}} \mathrm{b}^{2}}{\pi^{2}\left(\mathrm{D}_{11} \mathrm{D}_{22}\right)^{1 / 2}}=\mathrm{L}_{1} \tilde{\mathrm{p}}_{\mathrm{cr}} \\
& \mathrm{K}_{\mathrm{y}} \equiv\left(\mathrm{n}_{\mathrm{y} 1}\right)_{\mathrm{cr}}=\frac{\left(\mathrm{N}_{\mathrm{y} 1}\right)_{\mathrm{cr}} \mathrm{b}^{2}}{\pi^{2} \mathrm{D}_{22}}=\mathrm{L}_{2} \widetilde{\mathrm{p}}_{\mathrm{cr}} \\
& \mathrm{K}_{\mathrm{s}} \equiv\left(\mathrm{n}_{\mathrm{xy} 1}\right)_{\mathrm{cr}}=\frac{\left(\mathrm{N}_{\mathrm{xy} 1}\right)_{\mathrm{cr}} \mathrm{b}^{2}}{\pi^{2}\left(\mathrm{D}_{11} \mathrm{D}_{22}^{3}\right)^{1 / 4}}=\mathrm{L}_{3} \tilde{\mathrm{p}}_{\mathrm{cr}} \\
& \mathrm{K}_{\mathrm{b}} \equiv\left(\mathrm{n}_{\mathrm{b} 1}\right)_{\mathrm{cr}}=\frac{\left(\mathrm{N}_{\mathrm{b} 1}\right)_{\mathrm{cr}} \mathrm{b}^{2}}{\pi^{2}\left(\mathrm{D}_{11} \mathrm{D}_{22}\right)^{1 / 2}}=\mathrm{L}_{4} \tilde{\mathrm{p}}_{\mathrm{cr}}
\end{aligned}
$$

where the quantities enclosed in the parentheses with the subscript "cr" are critical values that correspond to buckling and $\tilde{\mathrm{p}}_{\mathrm{cr}}$ is the magnitude of the loading parameter at buckling. Positive values of the coefficients $K_{x}$ and $K_{y}$ correspond to uniform compression loads, and the coefficient $K_{s}$ corresponds to uniform positive shear. The direction of a positive shear stress resultant that acts on a plate is shown in Fig. 1. The coefficient $K_{b}$ corresponds to the specific inplane bending load distribution defined by the selected values of the parameters $\varepsilon_{0}$ and $\varepsilon_{1}$ (see Fig. 1).

The mathematical expression used in the variational analysis to represent the general off-center and skewed buckle pattern is given by

$$
\mathrm{w}_{N}(\xi, \eta)=\sum_{m=1}^{N}\left(A_{m} \sin \pi \xi+B_{m} \cos \pi \xi\right) \Phi_{m}(\eta)
$$

where $\xi=x / \lambda$ and $\eta=y / b$ are nondimensional coordinates, $w_{N}$ is the out-of-plane displacement field, and $A_{m}$ and $B_{m}$ are the unknown displacement amplitudes. In accordance with the Rayleigh-Ritz method, the basis functions $\Phi_{\mathrm{m}}(\eta)$ are required to satisfy the kinematic boundary conditions on the plate edges at $\eta=$ 0 and 1. For the simply supported plates, the basis functions used in the analysis are given by

$$
\Phi_{m}(\eta)=\sin m \pi \eta
$$

for values of $m=1,2,3, \ldots, N$. Similarly, for the clamped plates, the basis functions are given by 


$$
\Phi_{m}(\eta)=\cos (m-1) \pi \eta-\cos (m+1) \pi \eta
$$

For both boundary conditions, the two long edges of a plate are free to move in-plane.

Algebraic equations that govern the buckling behavior of infinitely long plates are obtained by substituting the series expansion for the buckling mode given by Eq. (22) into a nondimensionalized form of the second variation of the total potential energy and then computing the integrals appearing in the nondimensional second variation in closed form. The resulting equations constitute a generalized eigenvalue problem that depends on the aspect ratio of the buckle pattern $\lambda / \mathrm{b}$ (see Fig. 1) and the nondimensional parameters and nondimensional stress resultants defined herein. The smallest eigenvalue of the problem corresponds to buckling and is found by specifying a value of $\lambda / b$ and solving the corresponding generalized eigenvalue problem for its smallest eigenvalue. This process is repeated for successive values of

$\lambda \mathrm{b}$ until the overall smallest eigenvalue is found.

Results that were obtained from the analysis described herein for uniform compression, uniform shear, pure inplane bending (given by $\varepsilon_{0}=-1$ and $\varepsilon_{1}=1$ ), and various combinations of these mechanical loads have been compared with other results for isotropic, orthotropic, and anisotropic plates that were obtained by using other analysis methods. These comparisons are discussed in Refs. 1-3, and in every case the results described herein were found to be in good agreement with those obtained from other analyses. Likewise, results were obtained for isotropic and specially orthotropic plates that are subjected to a general linear distribution of axial load across the plate width and compared with results that were obtained by seven different authors (see Ref. 4). In every case, the agreement was good.

\section{Prebuckling Stress Resultants}

Symmetrically laminated plates that are subjected to uniform temperature fields and restrained against thermal expansion and contraction may develop internal mechanical loads that can cause buckling. These induced mechanical loads enter the analysis through the membrane constitutive equations. The standard form of these membrane constitutive equations for thin plates, that is based on classical laminated-plate theory, is found in Refs. 9 and 10 and is expressed in terms of membrane stiffness coefficients and fictitious thermal stress resultants. Following Ref. 7, the membrane constitutive equations are used in the present study in an inverted form that uses the overall laminate coefficients of thermal expansion and the membrane compliance coefficients. This form of the membrane constitutive equations for symmetrically laminated plates is given by

$$
\left\{\begin{array}{c}
u_{, x} \\
v_{, y} \\
u_{, y}+v_{, x}
\end{array}\right\}=\left[\begin{array}{lll}
a_{11} & a_{12} & a_{16} \\
a_{12} & a_{22} & a_{26} \\
a_{16} & a_{26} & a_{66}
\end{array}\right]\left\{\begin{array}{c}
N_{x} \\
N_{y} \\
N_{x y}
\end{array}\right\}+\left\{\begin{array}{c}
\alpha_{x} \\
\alpha_{y} \\
\alpha_{x y}
\end{array}\right\} \Theta_{0}
$$

where $u(x, y)$ and $v(x, y)$ are the prebuckling, inplane displacements in the $\mathrm{x}$ - and $\mathrm{y}$-coordinate directions (see Fig. 2), respectively; $\alpha_{x}, \alpha_{y}$, and $\alpha_{x y}$ are the overall laminate coefficients of thermal expansion; the subscripted a-terms are the plate membrane compliance coefficients; $\Theta_{0}$ is the magnitude of the uniform temperature change from a predetermined stress- and strainfree reference state; and commas followed by a subscript denote partial differentiation with respect to the coordinate associated with the subscript. For restrained thermal expansion and contraction problems, the plates are assumed to be supported and loaded such that the prebuckling stress field is uniform, that is, homogeneous. With this assumption, a compatible displacement field is obtained directly by integrating Eqs. (25). This integration yields

$$
\begin{aligned}
& u(x, y)=\left(a_{11} N_{x}+a_{12} N_{y}+a_{16} N_{x y}+\alpha_{x} \Theta_{0}\right) x+g_{1} y+g_{2} \\
& v(x, y)=\left(a_{12} N_{x}+a_{22} N_{y}+a_{26} N_{x y}+\alpha_{y} \Theta_{0}\right) y+g_{3} x+g_{4}
\end{aligned}
$$

and

$$
g_{1}+g_{3}=a_{16} N_{x}+a_{26} N_{y}+a_{66} N_{x y}+\alpha_{x y} \Theta_{01}
$$

Equations (25)-(28) can be used to determine the thermally induced mechanical loadings for several problems of practical interest. The problems consist of plates restrained against axial thermal expansion and contraction (see Ref. 5 or 6), plates restrained against transverse thermal expansion or contraction ( $y$-coordinate direction), and plates completely restrained against thermal expansion and contraction (see Ref. 7). In the present study, however, symmetrically laminated plates that are elastically restrained against thermal expansion and contraction are considered.

All the stress resultants in a given plate are induced by the elastically restrained thermal expansion or contraction and, when considered together, may be destabilizing. The induced stress resultants are proportional to the strains caused by expansions or contractions and shearing deformations of the plate and the resistance provided by the restraining medium. Typically, the elastic resistance of a restraining medium is simulated with linear springs and expressed in terms of the corresponding spring stiffnesses. In the present study, the elastic resis- 
tance of a restraining medium is represented approximately, and in a general manner, in terms of the overall compliance and thermal expansion coefficients of a homogeneous, anisotropic restraining medium. For this type of representation, the prebuckling stress state is assumed to be uniform, for all practical purposes. When the compliance of the restraining medium is relatively small (close to a rigid medium), the induced prebuckling stress state is expected to be nearly uniform and the approach presented herein for calculating buckling loads is expected to be a reasonable approximation. For more compliant restraining media, nonuniformities in the prebuckling stress state are expected to increase, and thus the approach presented herein for calculating buckling loads must be used with caution and considerable engineering judgement. However, it is apparent that there are several types of elastic support conditions that can be modeled accurately with the approach presented herein. One type of elastic support condition, that may be useful in laboratory structural testing, is described herein after the general formulation of the induced prebuckling stress state and buckling analysis are presented.

In the general formulation presented herein, the thermally induced inplane stress resultants are determined by effectively enforcing displacement compatibility and Newton's law of action and reaction at the interfaces between the plate and the restraining medium. In particular, the stresses in the plate and the restraining medium are assumed to be, for the most part, uniform and to have the same constant values. Moreover, elongation and shear of the plate are assumed to be balanced by contraction and shear of the restraining medium such that the total, average strain in the plate is the negative of the total, average strain in the part of the restraining medium providing the resistance. For a restraining medium like the one shown in Fig. 2, but of effectively infinite planar extent, the stresses produced by expansion or contraction and shear are self equilibrated and, as a result, the elastic resistance provided can be idealized, or approximated, as the result of a uniform stress and deformation state acting over a rectangular region surrounding the plate and with finite dimensions. The finite dimensions could be determined by appyling St. Venant's principle to determine the decay lengths of the self-equilibrated stresses.

Following the reasoning of the previous suppositions, the positive-valued (total) strains in the restraining medium are obtained from the equivalent of Eq. (25) for the restaining medium. Then, the strains in the restraining medium are replaced in the equivalent of Eq. (25) with the negative of the corresponding strains in the plate, given by the left-hand side of Eq. (25), to fulfill displacement compatibilty in an overall manner. With this approach, the positive-valued (total) strains in the plate, given by the left-hand side of Eq. (25), are ex- pressed in terms of the properties of the restraining medium as

$$
\left\{\begin{array}{c}
u_{x_{x}} \\
v_{y} \\
u_{, y}+v_{, x}
\end{array}\right\}=-\left[\begin{array}{ccc}
a_{11}^{R} & a_{12}^{R} & a_{16}^{R} \\
a_{11}^{R} & a_{22}^{R} & a_{26}^{R} \\
a_{16}^{R} & a_{26}^{R} & a_{66}^{R}
\end{array}\right]\left\{\begin{array}{c}
N_{x} \\
N_{y} \\
N_{x y}
\end{array}\right\}-\left\{\begin{array}{c}
\alpha_{x}^{R} \\
\alpha_{y}^{R} \\
\alpha_{x y}^{R}
\end{array}\right\} \Theta_{0}
$$

where the superscript $\mathrm{R}$ denotes a quantity associated with the restraining medium. It is reiterated, that the negative signs in Eq. (29) come from the supposition that a positive, expansional strain for the plate in the $x$ direction produces compressive stress in the $x$-direction, that corresponds to a negative value for $\mathrm{N}_{\mathrm{x}}$. In addition, the Poisson effect produces a tensile stress in the $y$ direction, that corresponds to a positive value for $\mathrm{N}_{y}$ A similar response is noted for a positive, expansional strain for the plate in the $y$-direction. For a positive shearing deformation, a negative shearing stress resultant acts on the restraining medium.

Enforcing compatibility at the interface between the plate and the restraining medium is manifested by eliminating the strains from Eqs. (25) and (29), which gives

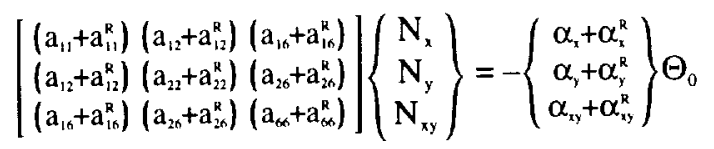

Next, because all the subcritical loads are zero-valued in the absence of applied mechanical loads, $N_{x}(x, y)=$ $-N_{x 1}^{c}, N_{y}(x, y)=-N_{y 1}$, and $N_{x y}(x, y)=N_{x y 1}$ (see Fig. 2). This substitution yields

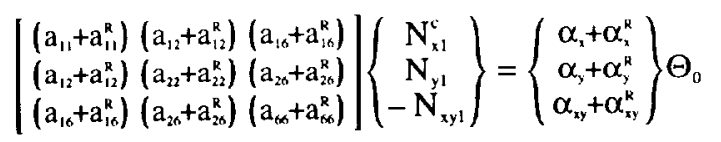

The thermally induced stress resultants for this problem are obtained by solving matrix Eq. (31) for $\mathrm{N}_{\mathrm{x} 1}^{\mathrm{c}}, \mathrm{N}_{\mathrm{y} 1}$, and $\mathrm{N}_{\mathrm{xyl}}$. The solution is given symbolically by

$$
\begin{aligned}
& N_{x 1}^{v}=\left[\bar{A}_{11}\left(\alpha_{1}+\alpha_{x}^{R}\right)+\bar{A}_{12}\left(\alpha_{y}+\alpha_{y}^{R}\right)+\bar{A}_{10}\left(\alpha_{x y}+\alpha_{x y}^{R}\right)\right] \Theta_{3,} \\
& N_{y 1}=\left[\bar{A}_{12}\left(\alpha_{x}+\alpha_{x}^{\mathrm{R}}\right)+\bar{A}_{22}\left(\alpha_{y}+\alpha_{y}^{\mathrm{R}}\right)+\bar{A}_{26}\left(\alpha_{3 x}+\alpha_{x y}^{\mathrm{R}}\right)\right] \Theta_{\mathrm{s}} \\
& N_{x y 1}=-\left[\bar{A}_{16}\left(\alpha_{x}+\alpha_{x}^{k}\right)+\bar{A}_{28}\left(\alpha_{y}+\alpha_{y}^{R}\right)+\bar{A}_{c o}\left(\alpha_{x y}+\alpha_{y y}^{R}\right)\right] \Theta_{,}
\end{aligned}
$$

where the subscripted $\overline{\mathrm{A}}$-terms are elements of the inverse of the matrix in the left-hand side of matrix Eq. (31). When the restraining medium is totally rigid, the compliances of the restraining medium in Eq. (31) are 
zero valued and the subscripted $\overline{\mathrm{A}}$-terms reduce to the usual membrane stiffnesses of laminated plate theory, which are denoted herein by subscripted A-terms. Because the elements of the matrix in the left-hand side of Eq. (31) typically have greater magnitude than the corresponding elements for only the plate, and because the elements of the inverse of a matrix are like their corresponding reciprocals, it follows that the subscripted $\bar{A}$-terms typically have smaller magnitude than the corresponding subscripted A-terms.

\section{Critical Temperature Change}

Equations (32)-(34) define a combined loading state that is induced by elastically restrained thermal expansion and contraction. More specifically, the equations indicate that the induced loads depend on the compliance of the restraining medium and the relative overall thermal expansion or contraction of the plate and the restraining medium. In addition, these equations show that each of the thermally induced mechanical loads generally depends on all three laminate coefficients of thermal expansion, and that positive values for the stress resultants are possible even for negative values of $\Theta_{0}$ (uniform cooling) and vice versa. For example, a laminate could have a negative value of $\alpha_{x}$ and still have a positive value of $\mathrm{N}_{\mathrm{x} 1}^{\mathrm{c}}$ (axial compression). Thus, the signs of $\Theta_{0}$ and the bracketed quantities in Eqs. (32)-(34) must be considered in formulating the buckling problem.

The buckling problem is posed by first substituting Eqs. (32)-(34) into Eqs. (10)-(12), respectively, to obtain expressions for the nondimensional stress resultants that are used to characterize the thermally induced mechanical loads. Following Ref. 7, the nondimensional stress resultants are expressed in terms of stiffness-weighted laminate thermal-expansion parameters denoted by $\alpha_{1}$, $\alpha_{2}$, and $\alpha_{3}$, and in terms of nondimensional compliance coefficients denoted by $\mathrm{C}_{1}, \mathrm{C}_{2}$, and $\mathrm{C}_{3}$. These expressions, with the use of Eqs. (14)-(16), are given by

$$
\begin{aligned}
& \mathrm{n}_{\mathrm{x} 1}^{\mathrm{c}}=\frac{12 \mathrm{~b}^{2}}{\pi^{2} \mathrm{t}^{2}} \frac{\alpha_{1}}{\left(1+\mathrm{C}_{1}\right)} \Theta_{0}=\mathrm{L}_{1} \tilde{\mathrm{p}} \\
& \mathrm{n}_{\mathrm{y} 1}=\frac{12 \mathrm{~b}^{2}}{\pi^{2} \mathrm{t}^{2}} \frac{\alpha_{2}}{\left(1+\mathrm{C}_{2}\right)} \Theta_{0}=\mathrm{L}_{2} \tilde{\mathrm{p}} \\
& \mathrm{n}_{\mathrm{xy} 1}=\frac{12 \mathrm{~b}^{2}}{\pi^{2} \mathrm{t}^{2}} \frac{\alpha_{3}}{\left(1+\mathrm{C}_{3}\right)} \Theta_{0}=L_{3} \tilde{\mathrm{p}}
\end{aligned}
$$

where

$$
\alpha_{1}=\frac{t^{2}\left(A_{11} \alpha_{x}+A_{12} \alpha_{y}+A_{16} \alpha_{x y}\right)}{12 \sqrt{D_{11} D_{22}}}
$$

$$
\begin{aligned}
& \alpha_{2}=\frac{t^{2}\left(A_{12} \alpha_{x}+A_{22} \alpha_{y}+A_{20} \alpha_{x y}\right)}{12 D_{22}} \\
& \alpha_{3}=-\frac{\mathfrak{t}^{2}\left(A_{16} \alpha_{x}+A_{26} \alpha_{y}+A_{x x} \alpha_{x y}\right)}{12\left(D_{11} D_{22}^{3}\right)^{1 / 4}} \\
& C_{1}=\frac{A_{11} \alpha_{x}+A_{12} \alpha_{y}+A_{1 x} \alpha_{k y}}{\bar{A}_{11}\left(\alpha_{x}+\alpha_{x}^{R}\right)+\bar{A}_{12}\left(\alpha_{y}+\alpha_{y}^{R}\right)+\bar{A}_{18}\left(\alpha_{x y}+\alpha_{x y}^{k}\right)}-1 \\
& C_{2}=\frac{A_{12} \alpha_{z}+A_{22} \alpha_{y}+A_{20} \alpha_{x y}}{\bar{A}_{12}\left(\alpha_{x}+\alpha_{x}^{\mathrm{R}}\right)+\bar{A}_{22}\left(\alpha_{y}+\alpha_{y}^{\mathrm{p}}\right)+\bar{A}_{26}\left(\alpha_{2 y}+\alpha_{y y}^{\mathrm{R}}\right)}-1 \\
& C_{3}=\frac{A_{10} \alpha_{x}+A_{2 n} \alpha_{y}+A_{6 x} \alpha_{x y}}{\bar{A}_{10}\left(\alpha_{x}+\alpha_{1}^{R}\right)+\bar{A}_{20}\left(\alpha_{y}+\alpha_{y}^{R}\right)+\bar{A}_{s x}\left(\alpha_{x y}+\alpha_{x y}^{k}\right)}-1
\end{aligned}
$$

It is important to point out that because the subscripted $\overline{\mathrm{A}}$-terms are typically less than the corresponding subscripted A-terms, that typically $\mathrm{C}_{1} \geq 0, \mathrm{C}_{2} \geq 0$, and $\mathrm{C}_{3} \geq 0$ when the thermal expansion or contraction of the restraining medium is negligible. Moreover, for a totally rigid restraining medium with negligible thermal expansion or contraction, $C_{1}=C_{2}=C_{3}=0$. However, in general, the compliance factors $\mathrm{C}_{1}, \mathrm{C}_{2}$, and $\mathrm{C}_{3}$ may be negative valued when the restraining medium has a higher relative thermal expansion or contraction than the plate. Thus, the relative stiffness and relative thermal expansion and contraction can significantly change the nature of the thermally induced mechanical loads, as expected.

Equations (10)-(12) and (35)-(37) indicate that $\mathrm{N}_{\mathrm{x} 1}^{\mathrm{c}}, \mathrm{N}_{\mathrm{yl}}$, and $\mathrm{N}_{\mathrm{xy} 1}$ are positive-valued (see Fig. 2b) when $\alpha_{1} /\left(1+C_{1}\right), \alpha_{2} /\left(1+C_{2}\right)$, and $\hat{\alpha}_{3} /\left(1+C_{3}\right)$ are positivevalued, respectively, and when $\Theta_{0}$ is positive-valued. Similarly, $\mathrm{N}_{\mathrm{x} 1}^{\mathrm{c}}, \mathrm{N}_{\mathrm{y} 1}$, and $\mathrm{N}_{\mathrm{xy} 1}$ are negative-valued when $\hat{\alpha}_{1} /\left(1+C_{1}\right), \hat{\alpha}_{2} /\left(1+C_{2}\right)$, and $\hat{\alpha}_{3} /\left(1+C_{3}\right)$ are positivevalued, respectively, and $\Theta_{0}$ is negative-valued, or when $\hat{\alpha}_{1} /\left(1+C_{1}\right), \alpha_{2} /\left(1+C_{2}\right)$, and $\alpha_{3} /\left(1+C_{3}\right)$ are negative valued, respectively, and $\Theta_{0}$ is positive-valued. Next, Eqs. (35)-(37) are substituted into Eqs. (18)-(20) to obtain the relationships between the mechanical-buckling coefficients, load factors, and the critical temperature change $\Theta_{0}^{\text {cr }}$; that is,

$$
\begin{aligned}
& K_{x} \equiv\left(n_{x 1}^{c}\right)_{c r}=\frac{12 b^{2}}{\pi^{2} t^{2}} \frac{\alpha_{1}}{\left(1+C_{1}\right)} \Theta_{0}^{c r}=L_{1} \widetilde{p}_{c r} \\
& K_{y} \equiv\left(n_{y 1}\right)_{c r}=\frac{12 b^{2}}{\pi^{2} t^{2}} \frac{\alpha_{2}}{\left(1+C_{2}\right)} \Theta_{0}^{c r}=L_{2} \widetilde{p}_{c r} \\
& K_{s} \equiv\left(n_{x y 1}\right)_{c r}=\frac{12 b^{2}}{\pi^{2} t^{2}} \frac{\alpha_{3}}{\left(1+C_{3}\right)} \Theta_{0}^{c r}=L_{3} \tilde{p}_{c r}
\end{aligned}
$$


where the critical eigenvalue $\tilde{p}_{\mathrm{cr}}=\tilde{\mathrm{p}}_{\mathrm{cr}}\left(\beta, \gamma, \delta, \mathrm{L}_{1}, \mathrm{~L}_{2}, \mathrm{~L}_{3}\right)$ for a given set of flexural (bending) boundary conditions (e.g., simply supported and clamped edges). Eqs. (44)(46) reduce to the corresponding equations presented in Ref. 7 for plates that are fully restrained against thermal expansion and contraction $\left(C_{1}=C_{2}=C_{3}=0\right)$. For an isotropic material, $\alpha_{3}=0$ and $\alpha_{1}=\alpha_{2}=\alpha(1+v)$, where $\alpha$ is the coefficient of thermal expansion and $v$ is Poisson's ratio of a homogeneous isotropic material.

The next step in posing the buckling problem is to define the load factors $\mathrm{L}_{1}, \mathrm{~L}_{2}$, and $\mathrm{L}_{3}$ that appear in Eqs. (35)-(37) and (44)-(46). It is important to reiterate that positive, negative, and zero values for $\hat{\alpha}_{1} \Theta_{1} /\left(1+C_{1}\right)$ correspond to positive, negative, and zero values for $\mathbf{N}_{\mathbf{x}}^{\mathrm{c}}$, respectively (see Fig. 2b). Similarly, positive, negative, and zero values for $\alpha_{2} \Theta_{\mathbb{N}} /\left(1+C_{2}\right)$ correspond to positive, negative, and zero values for $\mathrm{N}_{\mathrm{y} 1}$, respectively; and positive, negative, and zero values for $\alpha_{3} \Theta_{0} /\left(1+C_{3}\right)$ correspond to positive, negative, and zero values for $\mathrm{N}_{\mathrm{xy}}$, respectively. To define the load factors properly, the signs of $\mathrm{N}_{\mathrm{x} 1}^{\mathrm{c}}, \mathrm{N}_{\mathrm{y} 1}$, and $\mathrm{N}_{\mathrm{xy} 1}$ must be considered. Specifically, the load factors must be defined such that positive values of $L_{1}, L_{2}$, and $L_{3}$ correspond to positive values of $\mathrm{N}_{\mathrm{x} 1}^{\mathrm{c}}, \mathrm{N}_{\mathrm{y} 1}$, and $\mathrm{N}_{\mathrm{xy} 1}$, respectively. Moreover, both positive (heating) and negative (cooling) values of $\Theta_{0}$ must be considered. These requirements lead to six cases that must be considered in formulating the buckling analysis; that is, the cases for which $\alpha_{1} \Theta_{0} /\left(1+C_{1}\right)>0, \quad \alpha_{1} \Theta_{d} J\left(1+C_{1}\right)<0, \quad \hat{\alpha}_{1}=0$ with $\alpha_{2} \Theta_{N} \nu\left(1+C_{2}\right)>0, \quad \alpha_{1}=0$ with $\alpha_{2} \Theta_{0} d\left(1+C_{2}\right)<0$, $\alpha_{1}=\alpha_{2}=0$ with $\hat{\alpha}_{3} \Theta_{0} /\left(1+C_{3}\right)>0$, and $\alpha_{1}=\alpha_{2}=0$ with $\alpha_{3} \Theta_{0} /\left(1+C_{3}\right)<0$. The values of the mechanicalload factors $L_{1}, L_{2}$, and $L_{3}$ for each of these cases are presented subsequently.

Load factors for case 1 . For the case when $\hat{\alpha}_{1} \Theta_{0} /\left(1+C_{1}\right)>0, N_{x 1}^{c}>0$ and $L_{1}=1$ is appropriate (axial compression). The values for the other two load factors that are needed to completely define the prebuckling stress state are obtained by dividing Eqs. (19) and (20) by Eq. (18), with $\mathrm{L}_{1}=1$, or by dividing Eqs. (36) and (37) by Eq. (35). This step yields

$$
\begin{aligned}
L_{2}=\frac{N_{y 1}}{N_{41}^{c}}\left(\frac{D_{11}}{D_{22}}\right)^{1 / 2}=\left(\frac{1+C_{1}}{1+C_{2}}\right) \frac{\hat{\alpha}_{2}}{\alpha_{11}} \\
\left.=\left(\frac{1+C_{1}}{1+C_{2}}\right) \frac{A_{12} \alpha_{1}+A_{22} \alpha_{y}+A_{20} \alpha_{11} \alpha_{x}+A_{12} \alpha_{y}+A_{10} \alpha_{x y}}{\left(D_{11}\right.}\right)^{1.2}
\end{aligned}
$$

$$
\begin{aligned}
L_{3}=\frac{N_{x y 1}}{N_{11}^{c}}\left(\frac{D_{11}}{D_{22}}\right)^{1 / 4}=\left(\frac{1+C_{1}}{1+C_{3}}\right) \frac{\alpha_{1}}{\alpha_{1}} \\
=-\left(\frac{1+C_{1}}{1+C_{3}}\right) \frac{A_{18} \alpha_{x}+A_{28} \alpha_{y}+A_{\alpha x} \alpha_{x y}}{A_{11} \alpha_{1}+A_{12} \alpha_{y}+A_{10} \alpha_{x y}}\left(\frac{D_{11}}{D_{22}}\right)^{1 / 4}
\end{aligned}
$$

For an isotropic plate, these expressions reduce to $\mathrm{L}_{2}=\left(1+\mathrm{C}_{1}\right) /\left(1+\mathrm{C}_{2}\right)$ and $\mathrm{L}_{3}=0$. With $\mathrm{L}_{1}=1$ and $\mathrm{L}_{2}$ and $\mathrm{L}_{3}$ defined by Eqs. (47) and (48), the relationship between the critical value of the mechanical loading parameter $\widetilde{\mathrm{p}}_{\mathrm{cr}}$ and the critical temperature change $\Theta_{0}^{\text {cr }}$ is determined by Eq. (44); that is,

$$
\mathrm{K}_{\mathrm{x}}=\frac{12 \mathrm{~b}^{2}}{\pi^{2} \mathrm{t}^{2}} \frac{\alpha_{1}}{\left(1+\mathrm{C}_{1}\right)} \Theta_{0}^{\alpha}=\tilde{\mathrm{p}}_{\mathrm{cr}}
$$

where $\tilde{\mathrm{p}}_{\mathrm{cr}}=\tilde{\mathrm{p}}_{\mathrm{cr}}\left(\beta, \gamma, \delta, \mathrm{L}_{1}, \mathrm{~L}_{2}, \mathrm{~L}_{3}\right)$ for a given set of flexural boundary conditions. It is important to point out that Eq. (49) yields positive and negative values for $\Theta_{0}^{\text {cr }}$ for positive and negative values of $\hat{\alpha}_{1} /\left(1+C_{1}\right)$, respectively. Moreover, it is important to reiterate that the relationship between $\tilde{\mathrm{p}}_{\mathrm{cr}}$ and the corresponding mechanical buckling coefficients $\mathrm{K}_{\mathrm{x}}, \mathrm{K}_{\mathrm{y}}$, and $\mathrm{K}_{\mathrm{s}}$ is given by Eqs. (49), (45), and (46), respectively.

Load factors for case 2. For the case when $\alpha_{1} \Theta_{0} /\left(1+C_{1}\right)<0, N_{\times 1}^{c}<0$ and $L_{1}=-1$ is appropriate (axial tension). Like for the previous case, the values for the other two load factors that are needed to completely define the prebuckling stress state are obtained by dividing Eqs. (19) and (20) by Eq. (18), but with $\mathrm{L}_{1}=-1$, or by dividing Eqs. (36) and (37) by Eq. (35). This step yields

$$
\begin{aligned}
& \mathrm{L}_{2}=-\frac{\mathrm{N}_{\mathrm{y1}}}{\mathrm{N}_{\mathrm{xi}}^{-}}\left(\frac{\mathrm{D}_{11}}{\mathrm{D}_{22}}\right)^{1 / 2}=-\left(\frac{1+\mathrm{C}_{1}}{1+\mathrm{C}_{2}}\right) \frac{\hat{\alpha}_{2}}{\hat{\alpha}_{1}} \\
& =-\left(\frac{1+C_{1}}{1+C_{2}}\right) \frac{A_{12} \alpha_{x}+A_{22} \alpha_{y}+A_{26} \alpha_{2 y}}{A_{11} \alpha_{x}+A_{12} \alpha_{y}+A_{13} \alpha_{x y}}\left(\frac{D_{11}}{D_{22}}\right)^{12} \\
& \mathbf{L}_{3}=-\frac{\mathbf{N}_{x y 1}}{\mathbf{N}_{x 1}^{c}}\left(\frac{D_{11}}{D_{22}}\right)^{1 / 4}=-\left(\frac{1+C_{1}}{1+C_{3}}\right) \frac{\alpha_{3}}{\alpha_{1}} \\
& =\left(\frac{1+C_{1}}{1+C_{3}}\right) \frac{A_{10} \alpha_{x}+A_{20} \alpha_{y}+A_{x x} \alpha_{x y}}{A_{11} \alpha_{x}+A_{12} \alpha_{y}+A_{10} \alpha_{13}}\left(\frac{D_{11}}{D_{12}}\right)^{14}
\end{aligned}
$$

With $\mathrm{L}_{1}=-1$ and $\mathrm{L}_{2}$ and $\mathrm{L}_{3}$ defined by Eqs. (50) and (51), the relationship between the critical value of the mechanical loading parameter $\tilde{p}_{c r}$ and the critical temperature change $\Theta_{0}^{\mathrm{cr}}$ is again determined by Eq. (44); that is, 


$$
\mathrm{K}_{\mathrm{x}}=\frac{12 \mathrm{~b}^{2}}{\pi^{2} \mathrm{t}^{2}} \frac{\alpha_{1}}{\left(1+\mathrm{C}_{1}\right)} \Theta_{0}^{\mathrm{cr}}=-\tilde{\mathrm{p}}_{\mathrm{cr}}
$$

In contrast to the previous case, Eq. (52a) yields positive values for $\Theta_{0}^{\text {cr }}$ for negative values of $\alpha_{1} /\left(1+C_{1}\right)$, and vice versa. For laminates with $L_{3}=0$ (balanced laminates) and $L_{2} \leq 0$ (no transverse compression), no destabilizing compression or shear loads are present and buckling cannot occur because the plate is in a state of either axial or biaxial tension. In contrast, when $\mathrm{L}_{3}=0$ and $\mathrm{L}_{2}>0$, a plate is subjected to axial tension and transverse compression. Figures $29-31$ of Ref. 1 indicate that an infinitely long plate buckles as a wide column for this type of loading, and that the buckling coefficient $\mathrm{K}_{\mathrm{y}}=1$ and 4 for simply supported and clamped plates, respectively. With $\mathrm{K}_{\mathrm{y}}$ known, Eqs. (45) and (52a) give

$$
K_{x}=\frac{12 b^{2}}{\pi^{2} t^{2}} \frac{\alpha_{1}}{\left(1+C_{1}\right)} \Theta_{0}^{\alpha}=-\frac{K_{y}}{L_{2}}
$$

Load factors for case 3 . For a general symmetric laminate, the possibility exists that $\alpha_{1}=0$, which implies that $N_{x 1}^{c}=0$. For this case, $L_{1}=0$ is appropriate and the sign of $\alpha_{2} \Theta_{0} d\left(1+C_{2}\right)$ must be considered in defining the nonzero load factors. In particular, for $\alpha_{2} \Theta_{\mathcal{N}} /\left(1+\mathrm{C}_{2}\right)>0, \mathrm{~N}_{\mathrm{yl}}>0$ and $\mathrm{L}_{1}=0$ and $\mathrm{L}_{2}=1$ are appropriate (transverse compression). The value for the load factor $\mathrm{L}_{3}$ that is needed to completely define the prebuckling stress state is obtained by dividing Eq. (20) by Eq. (19), with $\mathrm{L}_{2}=1$, or by dividing Eqs. (37) by Eq. (36). This step yields

$$
\begin{aligned}
L_{3}=\frac{N_{\mathrm{xy}}}{N_{y 1}} & \left(\frac{D_{22}}{D_{11}}\right)^{1 / 4}=\left(\frac{1+C_{2}}{1+C_{3}}\right) \frac{\alpha_{3}}{\alpha_{2}} \\
& =-\left(\frac{1+C_{2}}{1+C_{3}}\right) \frac{A_{10} \alpha_{x}+A_{2 x} \alpha_{y}+A_{s x} \alpha_{x y}}{A_{12} \alpha_{x}+A_{22} \alpha_{y}+A_{20} \alpha_{x y}}\left(\frac{D_{22}}{D_{11}}\right)^{1 / 4}
\end{aligned}
$$

The relationship between $\mathrm{L}_{3}$ and the mechanical-buckling coefficients $K_{y}$ and $K_{s}$ is shown in Figs. 24-27 of Ref. 1. With $\mathrm{L}_{1}=0, \mathrm{~L}_{2}=1$, and $\mathrm{L}_{3}$ defined by Eq. (53), the relationship between the critical value of the mechanical loading parameter $\widetilde{\mathrm{p}}_{\mathrm{cr}}$ and the critical temperature change $\Theta_{0}^{\mathrm{cr}}$ is determined by Eq. (45); that is,

$$
\mathrm{K}_{y}=\frac{12 \mathrm{~b}^{2}}{\pi^{2} \mathrm{t}^{2}} \frac{\alpha_{2}}{\left(1+\mathrm{C}_{2}\right)} \Theta_{0}^{\mathrm{cr}}=\tilde{\mathbf{p}}_{\mathrm{a}}
$$

It is important to point out that Eq. (54a) yields positive and negative values for $\Theta_{0}^{\mathrm{cr}}$ for positive and negative values of $\alpha_{2} /\left(1+C_{2}\right)$, respectively. For laminates with $\mathrm{L}_{3}=0$ (balanced laminates) a plate is subjected to only transverse compression. Thus, an infinitely long plate buckles as a wide column for this type of loading, and the buckling coefficient $K_{y}=1$ and 4 for simply supported and clamped plates, respectively. With $\mathrm{K}_{\mathrm{y}}$ known, Eq. (45) gives

$$
\frac{12 b^{2}}{\pi^{2} t^{2}} \frac{\alpha_{2}}{\left(1+C_{2}\right)} \Theta_{0}^{\mathrm{cr}}=\mathrm{K}_{y}
$$

Load factors for case 4. For the case when $\alpha_{1}=0$ and $\alpha_{2} \Theta_{0}\left(1+C_{2}\right)<0, N_{\mathrm{y} 1}<0$ and $\mathrm{L}_{1}=0$ and $\mathrm{L}_{2}=$ -1 are appropriate (transverse tension). Like for the previous case, the value for the load factor $L_{3}$ that is needed to completely define the prebuckling stress state is obtained by dividing Eq. (20) by Eq. (19), with $\mathrm{L}_{2}=-1$, or by dividing Eqs. (37) by Eq. (36). This step yields

$$
\begin{aligned}
& \mathbf{L}_{3}=-\frac{N_{x y 1}}{N_{y 1}}\left(\frac{D_{22}}{D_{11}}\right)^{1 / 4}=-\left(\frac{1+C_{2}}{1+C_{3}}\right) \frac{\hat{\alpha}_{3}}{\hat{\alpha}_{2}} \\
&=\left(\frac{1+C_{2}}{1+C_{1}}\right) \frac{A_{10} \alpha_{x}+A_{20} \alpha_{y}+A_{x \alpha} \alpha_{x y}}{A_{12} \alpha_{1}+A_{22} \alpha_{y}+A_{20} \alpha_{x \gamma}}\left(\frac{D_{22}}{D_{11}}\right)^{1 / 4}
\end{aligned}
$$

Like the previous case, the relationship between $L_{3}$ and the mechanical-buckling coefficients $\mathrm{K}_{\mathrm{y}}$ and $\mathrm{K}_{\mathrm{s}}$ is shown in Figs. 24-27 of Ref. 1. With $L_{1}=0, L_{2}=-1$, and $L_{3}$ defined by Eq. (55), the relationship between the critical value of the mechanical loading parameter $\tilde{\mathrm{p}}_{\mathrm{cr}}$ and the critical temperature change $\Theta_{0}^{\text {cr }}$ is determined by Eq. (45); that is,

$$
\mathrm{K}_{\mathrm{y}}=\frac{12 \mathrm{~b}^{2}}{\pi^{2} \mathrm{t}^{2}} \frac{\alpha_{2}}{\left(1+\mathrm{C}_{2}\right)} \Theta_{0}^{\mathrm{cr}}=-\tilde{\mathrm{p}}_{\mathrm{ct}}
$$

For this case, Eq. (56) yields positive values for $\Theta_{i 1}^{\text {cr }}$ for negative values of $\alpha_{2} /\left(1+C_{2}\right)$, and vice versa. For laminates with $\mathrm{L}_{3}=0$ (balanced laminates), no destabilizing compression or shear loads are present and buckling cannot occur because the plate is in a state of uniaxial, transverse tension.

Load factors for case 5. For the case with $\alpha_{1}=\alpha_{2}=0$ and $\hat{\alpha}_{3} \Theta_{0} /\left(1+C_{3}\right)>0, N_{x 1}^{c}=N_{y_{1}}=0$ and $\mathrm{N}_{\mathrm{xy} 1}>0$, which implies that $\mathrm{L}_{1}=\mathrm{L}_{2}=0$, and that $\mathrm{L}_{3}=1$ is appropriate (positive shear loading). With $\mathrm{L}_{1}=0, \mathrm{~L}_{2}$ $=0$, and $\mathrm{L}_{3}=1$, the relationship between the critical val- 
ue of the mechanical loading parameter $\tilde{\mathrm{p}}_{\mathrm{cr}}$ and the critical temperature change $\Theta_{0}^{\mathrm{cr}}$ is determined by Eq. (46); that is,

$$
K_{s}=\frac{12 b^{2}}{\pi^{2} t^{2}} \frac{\alpha_{3}}{\left(1+C_{3}\right)} \Theta_{0}^{c r}=\tilde{p}_{s}
$$

Again, it is important to point out that Eq. (57) yields positive and negative values for $\Theta_{0}^{\text {cr }}$ for positive and negative values of $\alpha_{3} /\left(1+C_{3}\right)$, respectively. In addition, values of the mechanical-buckling coefficient $\mathrm{K}_{s}$ for several laminates are given in Ref. 1.

Load factors for case 6 . The final case to consider is when $\hat{\alpha}_{1}=\alpha_{2}=0$ and $\alpha_{3} \Theta_{0} /\left(1+C_{3}\right)<0$. For this case, $N_{x 1}^{c}=N_{y 1}=0$ and $N_{x y 1}<0$, which implies that $L_{1}$ $=L_{2}=0$, and that $L_{3}=-1$ is appropriate (negative shear loading). With $\mathrm{L}_{1}=0, \mathrm{~L}_{2}=0$, and $\mathrm{L}_{3}=-1$, the relationship between the critical value of the mechanical loading parameter $\tilde{p}_{\mathrm{cr}}$ and the critical temperature change $\Theta_{1)}^{\mathrm{cr}}$ is again determined by Eq. (46); that is,

$$
\mathrm{K}_{\mathrm{s}}=\frac{12 \mathrm{~b}^{2}}{\pi^{2} \mathrm{t}^{2}} \frac{\alpha_{3}}{\left(1+\mathrm{C}_{3}\right)} \Theta_{0}^{\mathrm{cr}}=-\tilde{\mathbf{p}}_{\mathrm{cr}}
$$

In contrast to the previous case, Eq. (58) yields positive values for $\Theta_{0}^{\text {cr }}$ for negative values of $\hat{\alpha}_{3} /\left(1+C_{3}\right)$, and vice versa.

Finally, it is important to mention that the approach used herein to define the prebuckling stress state and the critical temperature change $\Theta_{0}^{\text {cr }}$ also applies for a more sophisticated plate theory, like a first-order transverseshear deformation theory. For this theory, $\tilde{\mathrm{p}}_{\mathrm{cr}}$ would depend also upon additional nondimensional parameters that characterize the transverse-shear flexibility. Thus, the only difference in the results for the two plate bending theories is the actual value of $\tilde{\mathrm{p}}_{\mathrm{cr}}$ that is used in Eqs. (44)-(46), for a given problem. It is also important to point out that $\widetilde{\mathrm{p}}_{\mathrm{cr}}$ for an infinitely long plate does not depend on the parameter $\alpha_{\infty}$. This fact has been shown in Refs. 1-4.

\section{Example of Compliance Coefficients}

Before performing the buckling analysis described herein, expressions for the compliance coefficients defined by Eqs. (41)-(43) are needed. To demonstrate the formulation of the present study, the example of a practical, laboratory-type apparatus, that is depicted in Fig. 3, is presented in this section.
An idealized structural arrangement is shown in Fig. 3 in which a central balanced, symmetrically laminated plate is restrained against inplane movement by four generally different, balanced, symmetrically laminated plates. For this arrangement, the thermally induced loads consist of tension or compression loads, without shear. The plates that provide the elastic restraint are number sequentially from one to four in the figure. Each of the four restraining plates is subjected to a generally different temperature change, denoted by $\Theta_{0}^{(k)}(k=1,2,3$, and 4$)$, and is enclosed by an insulated boundary. In addition, the central plate is subjected to a temperature change, denoted by $\Theta_{0}$, where the superscript (5) has been dropped for simplicity. The restraining plates are supported to allow tangential movement at the rigid frame shown in the figure and at the interfaces with the central plate.

The compliance of the restraining medium is determined by first defining the elongations of each of the five plates. In particular, the axial elongation of plates 1,2 , and 5 are denoted by $\delta_{x}^{(1)}, \delta_{x}^{(2)}$, and $\delta_{x}^{(5)}$, respectively. Similarly, the transverse elongation of plates 3,4 , and 5 are denoted by $\delta_{y}^{(3)}, \delta_{y}^{(4)}$, and $\delta_{y}^{(5)}$, respectively. To satisfy compatibility, the elongations must satisfy the following equations

$$
\begin{aligned}
& \delta_{x}^{(1)}+\delta_{x}^{(2)}+\delta_{x}^{(5)}=0 \\
& \delta_{y}^{(3)}+\delta_{y}^{(4)}+\delta_{y}^{(5)}=0
\end{aligned}
$$

because the surrounding frame is rigid. These compatibility conditions state that the axial and transverse elongation or expansion of the central plate is balanced by the corresponding contraction of the restraining plates. These conditions are expressed in terms of strains by

$$
\begin{aligned}
& \frac{\mathrm{a}^{(1)}}{\mathrm{a}} \varepsilon_{x}^{(1)}+\frac{\mathrm{a}^{(2)}}{\mathrm{a}} \varepsilon_{x}^{(2)}+\varepsilon_{x}^{(5)}=0 \\
& \frac{\mathrm{b}^{(3)}}{\mathrm{b}} \varepsilon_{y}^{(3)}+\frac{\mathrm{b}^{(4)}}{\mathrm{b}} \varepsilon_{y}^{(4)}+\varepsilon_{y}^{(5)}=0
\end{aligned}
$$

where $\varepsilon_{\mathrm{x}}^{(\mathrm{k})}$ and $\varepsilon_{\mathrm{y}}^{(\mathrm{k})}(\mathrm{k}=1,2,3,4$, and 5) are given by the corresponding elongation divided by the corresponding length or width.

Next, the strains in each plate are expressed in terms of the stress resultants as follows

$$
\begin{aligned}
& \varepsilon_{x}^{(k)}=a_{1}^{(k)} N_{x}^{(k)}+a_{l 2}^{(k)} N_{y}^{(k)}+\alpha_{x}^{(k)} \Theta_{0}^{(k)} \\
& \varepsilon_{y}^{(k)}=a_{2}^{(k)} N_{x}^{(k)}+a_{2}^{(k)} N_{y}^{(k)}+\alpha_{y}^{(k)} \Theta_{0}^{(k)}
\end{aligned}
$$

where the superscript (k) identifies the quantity for a given plate. These expressions are simplified by noting that $\mathrm{N}_{\mathrm{x}}^{(3)}=\mathrm{N}_{\mathrm{x}}^{(4)}=0$ and $\mathrm{N}_{\mathrm{y}}^{(1)}=\mathrm{N}_{\mathrm{y}}^{(2)}=0$, and by applying Newton's law of action and reaction between adjoining 
plates, which gives $\mathrm{N}_{\mathrm{x}}^{(1)}=\mathrm{N}_{\mathrm{x}}^{(2)}=\mathrm{N}_{\mathrm{x}}^{(5)} \equiv \mathrm{N}_{\mathrm{x}} \quad$ and $\mathbf{N}_{y}^{(3)}=N_{y}^{(4)}=N_{y}^{(5)} \equiv N_{y}$. Substituting these expression for the stress resultants into Eqs. (63) and (64) and then the resulting expressions for the strains into Eqs. (61) and (62) gives

$$
\begin{aligned}
&\left(a_{11}+\frac{a^{(1)}}{a} a_{11}^{(1)}\right.\left.+\frac{a^{(2)}}{a} a_{11}^{(2)}\right) N_{x}+a_{12} N_{y}= \\
&-\left(\alpha_{x} \Theta_{0}+\frac{a^{(1)}}{a} \alpha_{x}^{(1)} \Theta_{0}^{(1)}+\frac{a^{(2)}}{a} \alpha_{x}^{(2)} \Theta_{0}^{(2)}\right) \\
& a_{12} N_{x}+\left(a_{22}+\frac{b^{(3)}}{b} a_{22}^{(3)}+\frac{b^{(4)}}{b} a_{22}^{(4)}\right) N_{y}= \\
&-\left(\alpha_{y} \Theta_{01}+\frac{b^{(3)}}{b} \alpha_{y}^{(3)} \Theta_{01}^{(3)}+\frac{b^{(4)}}{b} \alpha_{y}^{(4)} \Theta_{0}^{(4)}\right)
\end{aligned}
$$

where the superscript (5) that identifies the central plate has been dropped for convenience and consistency with the previously used notation presented herein. The desired form of these two equations is obtained by expressing the temperature change in the restraining plates in terms of the temperature change in the central plate by $\Theta_{0}^{(k)}=\tau^{(k)} \Theta_{0}(k=1,2,3$, and 4$)$, and by defining

$$
\begin{gathered}
a_{11}^{R}=\frac{a^{(1)}}{a} a_{11}^{(1)}+\frac{a^{(2)}}{a} a_{11}^{(2)} \\
a_{22}^{R}=\frac{b^{(3)}}{b} a_{22}^{(3)}+\frac{b^{(4)}}{b} a_{22}^{(4)} \\
\alpha_{x}^{R}=\frac{a^{(1)}}{a} \alpha_{x}^{(1)} \tau^{(1)}+\frac{a^{(2)}}{a} \alpha_{x}^{(2)} \tau^{(2)} \\
\alpha_{y}^{R}=\frac{b^{(3)}}{b} \alpha_{y}^{(3)} \tau^{(3)}+\frac{b^{(4)}}{b} \alpha_{y}^{(4)} \tau^{(4)}
\end{gathered}
$$

These expressions indicate that, in general, each plate can have a different uniform, isolated temperature field. When $\tau^{(k)}=0$ for all the restraining plates $(k=1,2,3$, and 4 ), the restraining medium does not undergo any thermal deformations.

The desired form of Eqs. (65) and (66) is given in matrix form by

$$
\left[\begin{array}{cc}
\left(a_{11}+a_{11}^{R}\right) & a_{12} \\
a_{12} & \left(a_{22}+a_{22}^{R}\right)
\end{array}\right]\left\{\begin{array}{l}
N_{x} \\
N_{y}
\end{array}\right\}=-\left\{\begin{array}{c}
\alpha_{x}+\alpha_{x}^{R} \\
\alpha_{y}+\alpha_{y}^{R}
\end{array}\right\} \Theta_{0}
$$

Next, because all the subcritical loads are zero-valued, $N_{x}(x, y)=-N_{x 1}^{c}, N_{y}(x, y)=-N_{y 1}$, and $N_{x y}(x, y)=N_{x y 1}=0$ (see Fig. 2b). Substitution of these expressions into Eq. (71) yields

$$
\left[\begin{array}{cc}
\left(a_{11}+a_{11}^{R}\right) & a_{12} \\
a_{12} & \left(a_{22}+a_{22}^{R}\right)
\end{array}\right]\left\{\begin{array}{l}
N_{x x}^{c} \\
N_{y 1}^{c}
\end{array}\right\}=\left\{\begin{array}{l}
\alpha_{x}+\alpha_{x}^{R} \\
\alpha_{y}+\alpha_{y}^{R}
\end{array}\right\} \Theta_{0}
$$

which is a degenerate form of Eq. (31). Equation (72) shows that the thermally induced loads depend on the compliance and thermal expansion or contraction of the central plate and the restraining plates. For Eq. (72), Eqs. (32) and (33) reduce to

$$
\begin{aligned}
& N_{x 1}^{c}=\left[\bar{A}_{11}\left(\alpha_{x}+\alpha_{x}^{R}\right)+\bar{A}_{12}\left(\alpha_{y}+\alpha_{y}^{R}\right)\right] \Theta_{0} \\
& N_{y 1}=\left[\bar{A}_{12}\left(\alpha_{x}+\alpha_{x}^{R}\right)+\bar{A}_{22}\left(\alpha_{y}+\alpha_{y}^{R}\right)\right] \Theta_{0}
\end{aligned}
$$

where the subscripted $\overline{\mathrm{A}}$-terms are elements of the inverse of the matrix in the left-hand side of matrix Eq. (72). Similarly, the expressions for the compliance coefficients $C_{1}$ and $C_{2}$ that are needed for a buckling analysis and given by Eqs. (41)and (42), respectively, reduce to

$$
\begin{aligned}
& C_{1}=\frac{A_{11} \alpha_{x}+A_{12} \alpha_{y}}{\bar{A}_{11}\left(\alpha_{x}+\alpha_{x}^{\mathrm{R}}\right)+\bar{A}_{12}\left(\alpha_{y}+\alpha_{y}^{\mathrm{R}}\right)}-1 \\
& C_{2}=\frac{A_{12} \alpha_{x}+A_{22} \alpha_{y}}{\bar{A}_{12}\left(\alpha_{x}+\alpha_{x}^{\mathrm{R}}\right)+\bar{A}_{22}\left(\alpha_{y}+\alpha_{y}^{\mathrm{R}}\right)}-1
\end{aligned}
$$

When the restraining medium is totally rigid, the compliances of the restraining medium in Eq. (72) are zero valued and the subscripted $\bar{A}$-terms reduce to the usual membrane stiffnesses of laminated plate theory, which are denoted herein by subscripted A-terms.

Examination of Eq. (72) indicates, as expected, that the Poisson-like lateral expansion or contraction of the restraining plates shown in Fig. 3 are totally independent; that is, this behavior is manifested by the absence of $\mathrm{a}_{12}^{R}$ terms in Eq. (72). For a homogeneous, orthotropic restraining medium similar to that depicted in Fig. 2, this interaction of Poisson effects would be present, along with self-equilibrated shearing stresses and slight nonuniformities in the $N_{x}(x, y)$ and $N_{y}(x, y)$ distributions within the central plate. For this type of restraining medium, a reasonable approximation to the thermally induced loads is given by

$$
\left[\begin{array}{l}
\left(a_{11}+a_{11}^{R}\right)\left(a_{12}+a_{12}^{R}\right) \\
\left(a_{12}+a_{12}^{R}\right)\left(a_{22}+a_{22}^{R}\right)
\end{array}\right]\left\{\begin{array}{l}
N_{x}^{c} \\
N_{y 1}
\end{array}\right\}=\left\{\begin{array}{l}
\alpha_{x}+\alpha_{x}^{R} \\
\alpha_{y}+\alpha_{y}^{R}
\end{array}\right\} \Theta_{0}
$$

where the compliances of the restraining medium could be estimated by subdividing the restraining medium into plates, neglecting the self-equilibrated shearing stresses, and applying a procedure similar to that used to determine the compliances for the structural arrangement shown in Fig. 3.

For the results that are presented subsequently in the present paper, it is convenient to introduce the relative-compliance ratios $R_{1}=a_{11}^{R} / a_{11}$ and $R_{2}=a_{22}^{R} / a_{22}$. 
Similarly, it is convenient to introduce the ratios of the coefficients of thermal expansion that are given by $T_{1}=\alpha_{x}^{R} / \alpha_{x}$ and $T_{2}=\alpha_{y}^{R} / \alpha_{y}$. Once a particular laminate is selected for investigation, the relevant properties of the restraining medium are specified in a relative manner, with respect to the central plate, by defining values for $R_{1}, R_{2}, T_{1}$, and $T_{2}$. With these ratios, the subscripted $\bar{A}$ terms, appearing in Eqs. (73)-(76), become

$$
\begin{gathered}
\bar{A}_{11}=\frac{A_{11}\left(1+R_{2}\right)\left(A_{11} A_{22}-A_{12}^{2}\right)}{\left(1+R_{1}\right)\left(1+R_{2}\right) A_{11} A_{22}-A_{12}^{2}} \\
\bar{A}_{12}=\frac{A_{12}\left(A_{11} A_{22}-A_{12}^{2}\right)}{\left(1+R_{1}\right)\left(1+R_{2}\right) A_{11} A_{22}-A_{12}^{2}} \\
\bar{A}_{22}=\frac{A_{22}\left(1+R_{1}\right)\left(A_{11} A_{22}-A_{12}^{2}\right)}{\left(1+R_{1}\right)\left(1+R_{2}\right) A_{11} A_{22}-A_{12}^{2}}
\end{gathered}
$$

In addition, the compliance coefficients given by Eqs. (75) and (76) become

$1+\mathrm{C}_{1}=$

$$
\frac{\left(A_{11} \alpha_{1}+A_{12} \alpha_{y}\right)\left[\left(1+R_{1}\right)\left(1+R_{2}\right) A_{11} A_{12}-A_{12}^{2}\right]}{\left[A_{11} \alpha_{1}\left(1+R_{2}\right)\left(1+T_{1}\right)+A_{12} \alpha_{y}\left(1+T_{2}\right)\right]\left(A_{11} A_{22}-A_{12}^{2}\right)}
$$

$1+\mathrm{C}_{2}=$

$$
\frac{\left(A_{12} \alpha_{x}+A_{22} \alpha_{y}\right)\left[\left(1+R_{1}\right)\left(1+R_{2}\right) A_{11} A_{12}-A_{12}^{2}\right]}{\left[A_{12} \alpha_{x}\left(1+T_{1}\right)+A_{22} \alpha_{y}\left(1+R_{1}\right)\left(1+T_{2}\right)\right]\left(A_{11} A_{22}-A_{12}^{2}\right)}
$$

Moreover,

$\frac{1+C_{1}}{1+C_{2}}=$

$\left(\frac{A_{11} \alpha_{x}+A_{12} \alpha_{y}}{A_{12} \alpha_{1}+A_{22} \alpha_{y}}\right)\left[\frac{A_{12} \alpha_{x}\left(1+T_{1}\right)+A_{22} \alpha_{y}\left(1+R_{1}\right)\left(1+T_{2}\right)}{A_{11} \alpha_{x}\left(1+R_{2}\right)\left(1+T_{1}\right)+A_{12} \alpha_{y}\left(1+T_{2}\right)}\right]$

\section{Results for Common Laminates and Discussion}

Results are presented in Figs. 4-17 that illustrate the effects of the inplane elastic resistance provided by the laboratory-type apparatus, previously described herein (see Fig. 3), on the restraint characteristics and buckling behavior of several common laminated plates subjected to a uniform temperature field. In particular, results are presented for several common balanced, symmetric laminates that are made of IM7/5260 graphite-bismaleimide material (see Table 1). The laminates include $[( \pm 45 / 0 /$ 90) $\left.]_{\mathrm{m}}\right]_{\mathrm{s}}$ quasi-isotropic laminates, $\left[\left( \pm 45 / 0_{2}\right)_{\mathrm{m}}\right]_{\mathrm{s}}$ and $[( \pm 45 /$ $\left.\left.90_{2}\right)_{\mathrm{m}}\right]_{\mathrm{s}}$ quasi-orthotropic laminates, and $\left[( \pm \theta)_{\mathrm{m}}\right]_{\mathrm{s}}$ angleply laminates, where a positive value of the lamina fiber angle $\theta$ is shown in Fig. 10 . The $\left[\left( \pm 45 / 0_{2}\right)_{\mathrm{m}}\right]_{\mathrm{s}}$ and $[( \pm 45 /$ $\left.\left.90_{2}\right)_{\mathrm{m}}\right]_{\mathrm{s}}$ laminates are described herein as quasi-orthotropic because of the presence of relatively small amounts of flexural anisotropy. All of the results are based on classical laminated-plate theory and the nominal ply thickness used in the calculations is 0.005 in.

The buckling behavior of the $\left[( \pm 45 / 0 / 90)_{\mathrm{m}}\right]_{\mathrm{s}}$, $\left[\left( \pm 45 / 90_{2}\right)_{\mathrm{m}}\right]_{\mathrm{s}},\left[\left( \pm 45 / 0_{2}\right)_{\mathrm{m}}\right]_{\mathrm{s}}$, and $\left[( \pm \theta)_{\mathrm{m}}\right]_{\mathrm{s}}$ laminates is governed by cases 1 through 4 , that are defined by Eqs. (47) through (56), because balanced, symmetric laminates that are restrained against inplane movement do not exhibit shear loads when subjected to a uniform temperature field. When $\alpha_{1} /\left(1+C_{1}\right)$ is nonzero (see cases 1 and 2), the key parameters for these laminates that are needed to determine their stability are $\alpha_{1} /\left(1+C_{1}\right)$ and $\left(\frac{1+C_{1}}{1+C_{2}}\right) \frac{\alpha_{2}}{\alpha_{1}}, \quad$ or equivalently, $\quad \hat{\alpha}_{1} /\left(1+C_{1}\right) \quad$ and $\alpha_{2} /\left(1+C_{2}\right)$. When $\alpha_{1} /\left(1+C_{1}\right)=0$, the key parameter is $\alpha_{2} /\left(1+C_{2}\right)$, as defined by cases 3 and 4 .

Results are presented in Figs. 4-6 that show the nondimensional compliance coefficient $\left(1+\mathrm{C}_{1}\right)\left(1+\mathrm{T}_{1}\right)$ for the $\left[( \pm 45 / 0 / 90)_{\mathrm{m}}\right]_{\mathrm{s}},\left[\left( \pm 45 / 90_{2}\right)_{\mathrm{m}}\right]_{\mathrm{s}}$, and $\left[\left( \pm 45 / 0_{2}\right)_{\mathrm{m}}\right]_{\mathrm{s}}$ balanced, symmetric laminates, respectively. Specifically, results are shown as a function of the relative-compliance ratio $R_{1}=a_{11}^{R} / a_{11}$, for discrete values of the relativecompliance ratio $R_{2}=a_{22}^{R} / a_{22}$ that range from 0 to 0.5 . For these results, the ratios of the coefficients of thermal expansion, that are given by $\mathrm{T}_{1}=\alpha_{\mathrm{x}}^{\mathrm{R}} / \alpha_{\mathrm{x}}$ and $T_{2}=\alpha_{y}^{R} / \alpha_{y}$, are equal; that is $T_{1}=T_{2}$. Values of $R_{1}=R_{2}$ $=0$ correspond to plate that is rigidly restrained against thermal expansion or contraction. Nonzero values of $T_{1}$ account for thermal expansion of the restraining medium. The parameter $\left(1+C_{1}\right)\left(1+T_{1}\right)$ is used in the figures because $C_{1}$ was found to be independent of the number of laminates plies (determined by the symbol $\mathrm{m}$ in the stacking sequence specification) and because Eq. (81) can be expressed in a manner that is independent of $T_{1}$ for the special case of $T_{1}=T_{2}$, that is,

$$
\begin{aligned}
& \left(1+C_{1}\right)\left(1+T_{1}\right)= \\
& \frac{\left(A_{11} \alpha_{x}+A_{12} \alpha_{4}\right)\left[\left(1+R_{1}\right)\left(1+R_{2}\right) A_{11} A_{22}-A_{12}^{2}\right]}{\left[A_{11} \alpha_{x}\left(1+R_{2}\right)+A_{12} \alpha_{8}\right]\left(A_{11} A_{22}-A_{12}^{2}\right)}
\end{aligned}
$$

Thus, graphs of $\alpha_{1}$, that are given in Ref. 7 for specific values of the stacking sequence number $\mathrm{m}$, can be used with the graphs of $\left(1+C_{1}\right)\left(1+T_{1}\right)$ provided herein to determine the buckling behavior for a large number of restraint configurations that include thermal deformations of the restaining plates. 
The results in Figs. 4-6 indicate a strong effect of laminate stacking sequence on the value of $\left(1+\mathrm{C}_{1}\right)(1+$ $\left.T_{1}\right)$, with the axially stiff $\left[\left( \pm 45 / 0_{2}\right)_{m}\right]_{s}$ laminates exhibiting the widest variation with variations in $R_{1}$ and $R_{2}$. Results for the transversely stiff $\left[\left( \pm 45 / 90_{2}\right)_{\mathrm{m}}\right]_{\mathrm{s}}$ laminates exhibit the least variation. Together, the results in Figs. 4-6 indicate that the parameter $\left(1+C_{1}\right)\left(1+T_{1}\right)$ is a positive-valued, linear function of $R_{1}$ and increases with increases in $R_{1}$ or $R_{2}$. Because $\left(1+C_{1}\right)\left(1+T_{1}\right)>0$ and $\alpha_{1}>0$ for these laminates (see Fig. 3 of Ref. 7), it follows that $\alpha_{1} /\left(1+C_{1}\right)>0$ as long as $\left(1+T_{1}\right)>0$. Thus, the buckling behavior of these laminates is governed by case 1 for uniform heating and by case 2 for uniform cooling when $\left(1+T_{1}\right)>0$, which typically includes most situations of practical importance.

Results are presented in Fig. 7 that show the nondimensional compliance coefficient ratio $\left(1+C_{1}\right) /\left(1+C_{2}\right)$ as a function of the relative-compliance ratio $R_{1}$, for discrete values of the relative-compliance ratio $R_{2}$ that range from 0 to 0.5 . These results are also for the case where the ratios of the coefficients of thermal expansion are equal $\left(\mathrm{T}_{1}=\mathrm{T}_{2}\right)$. Moreover, the results are also independent of the number of laminate plies and the value of $T_{1}$. The black solid lines, the gray solid lines, and the gray dashed lines in the figure are for the $\left[( \pm 45 / 0 / 90)_{\mathrm{m}}\right]_{\mathrm{s}}$, $\left[\left( \pm 45 / 0_{2}\right)_{\mathrm{m}}\right]_{\mathrm{s}}$, and $\left[\left( \pm 45 / 90_{2}\right)_{\mathrm{m}}\right]_{\mathrm{s}}$ laminates, respectively. The expression for $\left(1+C_{1}\right) /\left(1+C_{2}\right)$ that is obtained by simplying Eq. (83) accordingly is given by

$$
\frac{1+C_{1}}{1+C_{2}}=\left(\frac{A_{11} \alpha_{x}+A_{12} \alpha_{y}}{A_{12} \alpha_{x}+A_{22} \alpha_{y}}\right)\left(\frac{A_{12} \alpha_{x}+A_{22} \alpha_{y}\left(1+R_{1}\right)}{A_{11} \alpha_{x}\left(1+R_{2}\right)+A_{12} \alpha_{y}}\right]
$$

Corresponding values of $\hat{\alpha}_{2} / \hat{\alpha}_{1}$ that are needed to compute the parameter $\left(\frac{1+C_{1}}{1+C_{2}}\right) \frac{\alpha_{2}}{\alpha_{1}}$ are given in Fig. 4 of Ref. 7, for specific numbers of laminate plies, and are all positive valued.

The results in Fig. 7 also show a strong effect of laminate stacking sequence on the value of $\left(1+C_{1}\right) /(1+$ $\mathrm{C}_{2}$ ). The greatest sensitivity to variations in $\mathrm{R}_{1}$, as indicated by the slopes of the lines, is exhibited by the axially stiff $\left[\left( \pm 45 / 0_{2}\right)_{\mathrm{m}}\right]_{\mathrm{s}}$ laminates, followed by the $\left[( \pm 45 / 0 / 90)_{\mathrm{m}}\right]_{\mathrm{s}}$ quasi-isotropic laminates and then the transversely stiff $\left[\left( \pm 45 / 90_{2}\right)_{\mathrm{m}}\right]_{\mathrm{s}}$ laminates. This trend appears to be reversed with respect to variations in $R_{2}$. In addition, Eq. (85) and the results in Fig. 7 indicate that the ratio $\left(1+C_{1}\right) /\left(1+C_{2}\right)$ is a positive-valued linear function of $R_{1}$ for all three laminates. The values of $\left(1+C_{1}\right) /$ $\left(1+C_{2}\right)$ for the axially stiff $\left[\left( \pm 45 / O_{2}\right)_{m}\right]_{s}$ laminates increase with increases in $R_{1}$ or $R_{2}$. In contrast, the values of $\left(1+C_{1}\right) /\left(1+C_{2}\right)$ for the $\left[( \pm 45 / 0 / 90)_{m}\right]_{s}$ quasi-isotropic laminates increase with increases in $R_{1}$ and decrease with increases in $R_{2}$. Likewise, the values of $\left(1+C_{1}\right) /\left(1+C_{2}\right)$ for the transversely stiff $\left[\left( \pm 45 / 90_{2}\right)_{m}\right]_{s}$ laminates decrease with increases in $\mathrm{R}_{1}$ or $\mathrm{R}_{2}$.

Results that are similar to those presented in Figs. 4-7 are presented in Figs. 8 and 9 for the special case where the relative-compliance ratios are equal and the ratios of the coefficients of thermal expansion are equal; that is $R_{1}=R_{2}$ and $T_{1}=T_{2}$. The black solid lines, the gray solid lines, and the gray dashed lines in the figures are for the $\left[( \pm 45 / 0 / 90)_{\mathrm{m}}\right]_{\mathrm{s}},\left[\left( \pm 45 / 0_{2}\right)_{\mathrm{m}}\right]_{\mathrm{s}}$, and $\left[\left( \pm 45 / 90_{2}\right)_{\mathrm{m}}\right]_{\mathrm{s}}$ laminates, respectively. Additionally, the results are independent of the number of laminate plies and the results in Fig. 9 are also independent of the value of $T_{1}$.

Like the results in Figs. 4-6, the results in Fig. 8 also show a strong effect of laminate stacking sequence on the value of $\left(1+C_{1}\right)\left(1+T_{1}\right)$ for the axially stiff $\left[\left( \pm 45 / 0_{2}\right)_{\mathrm{m}}\right]_{\mathrm{s}}$ laminates. The results for these axially stiff laminates exhibit the greatest variations in $\left(1+C_{1}\right)(1+$ $T_{1}$ ) with variations in $R_{1}$ and a slight nonlinearity in the curve. The results for the $\left[( \pm 45 / 0 / 90)_{m}\right]_{\mathrm{s}}$ quasi-isotropic laminates and the transversely stiff $\left[\left( \pm 45 / 90_{2}\right)_{\mathrm{m}}\right]_{\mathrm{s}}$ laminates exhibit the least variation with $R_{1}$ and are nearly identical. For all three laminate types, the parameter ( 1 $\left.+C_{1}\right)\left(1+T_{1}\right)$ is a positive-valued, monotonically increasing function of the relative-compliance ratio $\mathrm{R}_{1}$.

The results in Fig. 9 also show a strong effect of laminate stacking sequence on the value of $\left(1+\mathrm{C}_{1}\right) /(1+$ $\mathrm{C}_{2}$ ), like the results in Fig. 7. The greatest sensitivity to variations in $R_{1}$ is exhibited by the axially stiff $\left[\left( \pm 45 / 0_{2}\right)_{m}\right]_{s}$ laminates, which exhibit a slightly nonlinear, monotonically increasing variation with $R_{1}$. The next greatest variation in $\left(1+C_{1}\right) /\left(1+C_{2}\right)$ with $R_{1}$ is exhibited by the transversely stiff $\left[\left( \pm 45 / 90_{2}\right)_{m}\right]_{s}$ laminates, which exhibit a slightly nonlinear, monotonically decreasing variation with $R_{1}$. The least sensitivity to variations in $R_{1}$ is exhibited by the $\left[( \pm 45 / 0 / 90)_{m}\right]_{s}$ quasiisotropic laminates, for which $\left(1+C_{1}\right) /\left(1+C_{2}\right)=1$ for all values of $R_{1}$. This constant value of unity is seen by examining Eq. (85) and noting that $A_{11}=A_{22}$ and $\alpha_{x}=\alpha_{y}$ for this family of laminates.

In Ref. 7, it is shown that the values of $\alpha_{1}$ and $\hat{\alpha}_{2} / \alpha_{1}$ are all positive for the $\left[( \pm 45 / 0 / 90)_{\mathrm{m}} \mathrm{l}_{\mathrm{s}},\left[\left( \pm 45 / 0_{2}\right)_{\mathrm{m}}\right]_{\mathrm{s}}\right.$, and $\left[\left( \pm 45 / 90_{2}\right)_{m}\right]_{s}$ laminates. Likewise, the results in Figs. 4-9 indicate that $\left(1+C_{1}\right) /\left(1+C_{2}\right)>0$ and that $(1+$ $\left.C_{1}\right)>0$ as long as $\left(1+T_{1}\right)>0$. Thus, for this practical range of $T_{1}$, these laminates are loaded by biaxial compression when uniformly heated and by biaxial tension when uniformly cooled. Therefore, these laminates can 
buckle only when subjected to uniform heating (see case 1) when $\left(1+T_{1}\right)>0$.

Results are presented in Figs. 10 and 11 that show the stiffness-weighted thermal expansion parameters, $\frac{Q_{1}}{\left(1+C_{1}\right)\left(1+T_{1}\right)}$ and $\frac{Q_{2}}{\left(1+C_{2}\right)\left(1+T_{1}\right)}$, respectively, for the $\left[( \pm \theta)_{\mathrm{m}}\right]_{\mathrm{s}}$ angle-ply laminates. The results in these figures are presented as a function of the fiber angle $\theta$ and are for the special case where the relative-compliance ratios are equal and the ratios of the coefficients of thermal expansion are equal $\left(R_{1}=R_{2}\right.$ and $\left.T_{1}=T_{2}\right)$. The black solid lines correspond to different values of the relative-compliance ratio $R_{1}$, that range from values of 0 to 0.5 . Additionally, the results are independent of the number of laminate plies. An expression for $\frac{Q_{1}}{\left(1+C_{1}\right)\left(1+T_{1}\right)}$ is obtained by combining Eqs. (38) and (81) and then simplying. The result is given by

$$
\frac{\alpha_{1}}{\left(1+C_{1}\right)\left(1+T_{1}\right)}=\frac{t^{2}\left[A_{11} \alpha_{x}\left(1+R_{1}\right)+A_{12} \alpha_{y}\right]\left(A_{11} A_{22}-A_{2}^{2}\right)}{12 \sqrt{D_{11} D_{22}}\left[\left(1+R_{1}\right)^{2} A_{11} A_{22}-A_{12}^{2}\right]}
$$

Similarly, combining Eqs. (39) and (82) and then simplying gives

$$
\frac{\widehat{\alpha}_{2}}{\left(1+C_{2}\right)\left(1+T_{1}\right)}=\frac{\mathfrak{t}^{2}\left[A_{12} \alpha_{1}+A_{22} \alpha_{y}\left(1+R_{1}\right)\right]\left(A_{11} A_{22}-A_{22}^{2}\right)}{12 D_{22}\left[\left(1+R_{1}\right)^{2} A_{11} A_{22}-A_{12}^{2}\right]}
$$

The curves in Figs. 10 and 11 show a very strong dependence on the fiber angle $\theta$. In particular, $\frac{\alpha_{1}}{\left(1+C_{1}\right)\left(1+T_{1}\right)}$ is shown to increase, for the most part, with increasing values of $\theta$, and $\frac{a_{2}}{\left(1+C_{2}\right)\left(1+T_{1}\right)}$ is shown to decrease. In addition, both stiffness-weighted thermal expansion parameters are shown to decrease with increasing values of the relative-compliance ratio $R_{1}$. For the curves that correspond to $R_{1}=0,0.1,0.2$, and 0.3 ; both stiffness-weighted thermal expansion parameters are positive for all values of the fiber angle $\theta$, which means that $\hat{\alpha}_{1} /\left(1+C_{1}\right)>0$ as long as $\left(1+T_{1}\right)>0$. For these values of $R_{1}$, and for $\left(1+T_{1}\right)>0$, the laminates are loaded by biaxial compression for uniform heating (case 1) and biaxial tension for uniform cooling (case 2). Thus, buckling can occur only for uniform heating, as governed by case 1 .

The results for the angle-ply laminates with $R_{1}=$ 0.4 and 0.5 that are shown in Figs. 10 and 11 indicate the possibility of several thermally induced loading conditions, depending on the particular value of the fiber angle $\theta$. First, the curves that correspond to $R_{1}=0.4$ and 0.5 , have zero values for $\frac{a_{1}}{\left(1+C_{1}\right)\left(1+T_{1}\right)}$ that are indicated in
Fig. 10, by the unfilled circular symbols. Specifically, the curve that corresponds to $R_{1}=0.4$ has zero values at approximately $\theta=18 \mathrm{deg}$ and $29 \mathrm{deg}$. The curve that corresponds to $R_{1}=0.5$ has zero values at approximately $\theta=15 \mathrm{deg}$ and $32 \mathrm{deg}$. For these specific laminate and restraint configurations with $\frac{Q_{1}}{\left(1+C_{1}\right)\left(1+T_{1}\right)}=0$, Fig. 11 indicates that the corresponding values of $\frac{Q_{2}}{\left(1+C_{2}\right)\left(1+T_{1}\right)}$ are positive as long as $\left(1+T_{1}\right)>0$, which means that the plates are loaded by only transverse compression or tension for uniform heating or uniform cooling, respectively. Thus, the plates may buckle only under uniform heating, into a wide-column mode, as governed by Eq. (54b) of case 3. Second, the curve that corresponds to $R_{1}$ $=0.4$ has positive values for $\frac{\alpha_{1}}{\left(1+C_{1}\right)\left(1+T_{1}\right)}$ and $\frac{a_{2}}{\left(1+C_{2}\right)\left(1+T_{1}\right)}$ for values of (approximately) $\theta<18 \mathrm{deg}$, $29<\theta<61 \mathrm{deg}$, and $\theta>72 \mathrm{deg}$ as long as $\left(1+\mathrm{T}_{1}\right)>0$. Third, $\frac{Q_{1}}{\left(1+C_{1}\right)\left(1+T_{1}\right)}$ is negative and $\frac{Q_{2}}{\left(1+C_{2}\right)\left(1+T_{1}\right)}$ is positive for $18 \leq \theta \leq 29 \mathrm{deg}$, and $\frac{a_{1}}{\left(1+C_{1}\right)\left(1+T_{1}\right)}$ is positive and $\frac{\partial_{2}}{\left(1+C_{2}\right)\left(1+T_{1}\right)}$ is negative for $61 \leq \theta \leq 72 \mathrm{deg}$ as long as $(1$ $\left.+\mathrm{T}_{1}\right)>0$. Thus, for the range $\theta<18 \mathrm{deg}, 29<\theta<61$ $\mathrm{deg}$, and $\theta>72 \mathrm{deg}$; the laminates are loaded by biaxial compression for uniform heating (case 1) and biaxial tension for uniform cooling (case 2) and, thus, buckling can occur only for uniform heating. For $18 \leq \theta \leq 29 \mathrm{deg}$, the laminates are loaded by axial tension and transverse compression for uniform heating (case 2) and oppositely directed loads for uniform cooling (case 1). Thus, for 18 $\leq \theta \leq 29 \mathrm{deg}$, buckling can occur for uniform heating or cooling. For $61 \leq \theta \leq 72 \mathrm{deg}$, the laminates are loaded by axial compression and transverse tension for uniform heating (case 1) and oppositely directed loads for uniform cooling (case 2). Thus, for $61 \leq \theta \leq 72 \mathrm{deg}$, buckling can also occur for uniform heating or cooling.

The results in Figs. 10 and 11 for the $\left[( \pm \theta)_{m}\right]_{s}$ angle-ply laminates with $R_{1}=0.5$ and $\left(1+T_{1}\right)>0$ indicate behavioral trends that are similar to those previously described for the laminates with $R_{1}=0.4$. In particular, for the range $\theta<15 \mathrm{deg}, 32<\theta<58 \mathrm{deg}$, and $\theta>75 \mathrm{deg}$; the laminates are loaded by biaxial compression for uniform heating (case 1) and biaxial tension for uniform cooling (case 2), and buckling can occur only for uniform heating. For $15 \leq \theta \leq 32 \mathrm{deg}$, the laminates arc loaded by axial tension and transverse compression for uniform heating (case 2) and oppositely directed loads for uniform cooling (case 1). Thus, for $15 \leq \theta \leq 32 \mathrm{deg}$, buckling can occur for uniform heating or cooling. For 
$58 \leq \theta \leq 75 \mathrm{deg}$, the laminates are loaded by axial compression and transverse tension for uniform heating (case 1) and oppositely directed loads for uniform cooling (case 2). Thus, for $58 \leq \theta \leq 75 \mathrm{deg}$, buckling can occur for uniform heating or cooling.

Values for the critical temperature changes for the $\left[( \pm 45 / 0 / 90)_{\mathrm{m}}\right]_{\mathrm{s}},\left[\left( \pm 45 / 0_{2}\right)_{\mathrm{m}}\right]_{\mathrm{s}}$, and $\left[\left( \pm 45 / 90_{2}\right)_{\mathrm{m}}\right]_{\mathrm{s}}$ laminates and for the $\left[( \pm \theta)_{m}\right]_{s}$ laminates with $m=1$ and $m>5$ are shown in Figs. 12-17. In these figures, the buckling results are based on the solutions for infinitely long plates and uniform heating. The solid and dashed curves correspond to results for plates with simply supported and clamped flexural boundary conditions, respectively. In particular, Fig. 12 shows values of $\frac{12 \mathrm{~b}^{2}}{\pi^{2} \mathrm{t}^{2}} \widetilde{\Theta}_{0}^{\text {cr }}$ for the $[( \pm 45 /$ $\left.0 / 90)_{\mathrm{m}}\right]_{\mathrm{s}},\left[\left( \pm 45 / \mathrm{O}_{2}\right)_{\mathrm{m}}\right]_{\mathrm{s}}$, and $\left[\left( \pm 45 / 90_{2}\right)_{\mathrm{m}}\right]_{\mathrm{s}}$ laminates, that are fully (rigidly) restrained against thermal expansion or contraction, as a function of the number of laminate plies. This fully restrained condition is indicated by the symbol $\widetilde{\Theta}_{0}^{\text {cr }}$. Similarly, Fig. 13 shows values of $\frac{12 b^{2}}{\pi^{2} t^{2}} \widetilde{\Theta}_{0}^{\text {cr }}$ for the $\left[( \pm \theta)_{m}\right]_{s}$ laminates, with $m=1$ and $m>5$, that are fully (rigidly) restrained against thermal expansion or contraction. The results in Figs. 14-16 show the criticaltemperature-change ratio $\frac{\Theta \& r}{\Theta_{0}\left(1+C_{1}\right)}$ as a function of the nondimensional compliance ratio $\left(1+\mathrm{C}_{1}\right) /\left(1+\mathrm{C}_{2}\right)$ and the stacking sequence numbers $\mathrm{m}=1$ and $\mathrm{m}=8$ for the $\left[( \pm 45 / 0 / 90)_{m}\right]_{s},\left[\left( \pm 45 / 0_{2}\right)_{m}\right]_{s}$, and $\left[\left( \pm 45 / 90_{2}\right)_{m}\right]_{s}$ laminates, respectively. Likewise, the results in Fig. 17 show the critical-temperature-change ratio $\frac{\Theta \oint_{0}^{\mathrm{r}}}{\Theta_{0}^{\mathrm{cr}}\left(1+\mathrm{C}_{1}\right)}$ as a function of the nondimensional compliance ratio $\left(1+\mathrm{C}_{1}\right) /(1$ $\left.+C_{2}\right)$ for the $\left[( \pm \theta)_{m}\right]_{s}$ laminates, with $m>5$, and for values of the fiber angle $\theta=30,45$, and $60 \mathrm{deg}$. In Figs. 14-17, the results are based on the presumption that $(1+$ $\left.C_{1}\right)>0$, such that $\hat{\alpha}_{1} \Theta_{0} J\left(1+C_{1}\right)>0$ and the buckling responses are governed by case $1\left(\mathrm{~L}_{1}=1\right.$ and $\left.\mathrm{L}_{3}=0\right)$ for uniform heating. This presumption is reasonable because $\alpha_{1}$ and $\alpha_{2} / \alpha_{1}$ are all positive for these laminates, which means that $\left(\frac{1+C_{1}}{1+C_{2}}\right) \frac{Q_{2}}{Q_{1}}>0$ for the range of $\left(1+C_{1}\right)$ / $\left(1+\mathrm{C}_{2}\right)$ selected for the independent variable. Moreover, the parameter $\left(1+C_{1}\right)$ appears in the expression $\frac{\Theta_{\delta}^{\phi^{r}}}{\bar{\Theta}_{0}^{\mathrm{cr}}\left(1+\mathrm{C}_{1}\right)}$ as a value to be selected.

The results in Figs. 14-17 give the critical temperature change for a wide range of restraint conditions, relative to the critical temperature change for the corresponding plate that is fully (rigidly) restrained against thermal expansion or contraction (Figs. 12 and 13). For example, values of $\left(1+C_{1}\right)$ and $\left(1+C_{1}\right) /(1+$
$\left.\mathrm{C}_{2}\right)$ for the $\left[( \pm 45 / 0 / 90)_{m}\right]_{\mathrm{s}}$ quasi-isotropic laminates can be obtained from Figs. 4 and 7, respectively, and used with Fig. 14 to obtain critical temperature changes for a very large number of restraint configurations that are defined by the relative-compliance parameters $R_{1}$ and $R_{2}$ and by the ratio of the coefficients of thermal expansion given by $T_{1}$. This approach was used in the present study because the results for a given fully restrained plate serve as a reasonable reference point for assessing the effects of a given elastic restraining medium.

The buckling results shown in Figs. 12 and 13 for the fully restrained laminates were obtained by computing the ratios $\hat{\alpha}_{2} / \hat{\alpha}_{1}$ first, in accordance with the procedure of case 1. Next, the load factor $\mathrm{L}_{2}$ was determined by using Eq. (47) with $C_{1}=0$ and $C_{2}=0$. The load factor $L_{3}$ is zero-valued, because balanced laminates do not induce shear loads when subjected to restrained thermal expansion or contraction. Then, the critical value of the loading parameter $\tilde{\mathrm{p}}_{\mathrm{cr}}$ was determined for each of the laminates and the critical temperature change was obtained by using Eq. (49) with $C_{1}=0$. Equations (47) and (49) were used because $\hat{\alpha}_{1} \Theta_{0}>0$ for these laminates when subjected to uniform heating. For these laminates, the critical value of the loading parameter depends on the flexural boundary conditions, the plate flexural orthotropy and flexural anisotropy, and the value of the load factor $L_{2}$, which depends on the stiffness-weighted laminate thermal-expansion parameters. The stiffness-weighted laminate thermal-expansion parameters depend on the plate membrane orthotropy and the plate stiffnesses associated with pure bending action.

For the buckling results in Figs. 14-17, the nondimensional compliance ratio $\left(1+\mathrm{C}_{1}\right) /\left(\mathbf{I}+\mathrm{C}_{2}\right)$ was treated as an independent variable. For a fixed value of this ratio, buckling results were obtained by again computing the ratios $\alpha_{2} / \alpha_{1}$ first and then the load factor $L_{2}$ by using Eq. (47), in accordance with the procedure of case 1. Then, the critical value of the loading parameter $\tilde{p}_{c r}$ was determined from a buckling analysis for each of the laminates and the critical temperature change was obtained by using Eq. (49) in the form

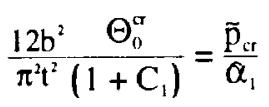

where $\tilde{\mathrm{p}}_{\mathrm{cr}}=\tilde{\mathrm{p}}_{\mathrm{cr}}\left(\beta, \gamma, \delta, L_{2}\right)$ for a given set of flexural boundary conditions and the load factor $\mathrm{L}_{2}$ depends on the stiffness-weighted laminate thermal-expansion parameters and the nondimensional compliance ratio $\left(1+C_{1}\right) /\left(1+C_{2}\right)$. Next, results obtained from Eq. (88) 
were divided by the corresponding results for the corresponding laminates that are fully restrained $\left(C_{1}=C_{2}=\right.$ 0 ), which are given symbolically by

$$
\frac{12 b^{2}}{\pi^{2} t^{2}} \widetilde{\Theta}_{0}^{\sigma r}=\frac{\widetilde{p}_{\alpha r}^{0}}{\alpha_{1}}
$$

Here, it is reiterated that the fully restrained condition is indicated by the symbol $\tilde{\Theta}_{0}^{\mathrm{cr}}$. Similarly, the symbol $\tilde{\mathrm{p}}_{\mathrm{cr}}^{0}$ is used to denote the critical value of the loading parameter for a given fully restrained laminate. For this situation, $\tilde{\mathrm{p}}_{\mathrm{cr}}^{0}=\tilde{\mathrm{p}}_{\mathrm{cr}}^{0}\left(\beta, \gamma, \delta, L_{2}^{0}\right)$, where $L_{2}^{0}=\alpha_{2} / \alpha_{1}$. Finally, dividing Eqs. (88) and (89) gives the result

$$
\frac{\Theta_{1}^{\alpha}}{\Theta_{0}^{c}\left(1+C_{l}\right)}=\frac{\tilde{p}_{\alpha}}{\tilde{p}_{\alpha}^{0}}
$$

It is useful to point out, that when $\tilde{\mathrm{p}}_{\mathrm{cr}}$ and $\tilde{\mathrm{p}}_{\mathrm{cr}}^{0}$ correspond to wide-column buckling modes, Eq. (90) reduces to

$$
\frac{\Theta_{0}^{\pi}}{\bar{\Theta}_{0}^{\mathrm{r}}\left(1+\mathrm{C}_{1}\right)}=\left(\frac{1+\mathrm{C}_{1}}{1+\mathrm{C}_{2}}\right)^{-1}
$$

This special case is indicated in Figs. 14-17 by the solid gray curve in each figure (the solid gray curve lies underneath the solid black curve in Fig. 14).

The results shown in Fig. 12 for the fully restrained plates indicate that the critical temperature change $\frac{12 b^{2}}{\pi^{2} t^{2}} \widetilde{\Theta}_{0}^{\text {cr }}$ is highly dependent on the arrangement of the 45-deg, 0-deg, and 90-deg plies. As the number of plies increases, the magnitude of the critical temperature change decreases for the clamped and simply supported $\left[\left( \pm 45 / 0_{2}\right)_{\mathrm{m}}\right]_{\mathrm{s}}$ laminates and increases for the clamped and simply supported $\left[( \pm 45 / 0 / 90)_{\mathrm{m}}\right]_{\mathrm{s}}$ laminates and the simply supported $\left[\left( \pm 45 / 90_{2}\right)_{m}\right]_{\mathrm{s}}$ laminates. The critical temperature change varies only slightly for the clamped $\left[\left( \pm 45 / 90_{2}\right)_{\mathrm{m}}\right]_{\mathrm{s}}$ laminates. Overall, the plates with clamped edges are more buckling resistant than the corresponding plates with simply supported edges, as expected. The clamped and simply supported $\left[\left( \pm 45 / 90_{2}\right)_{\mathrm{m}}\right]_{\mathrm{s}}$ laminates require the most heating to cause buckling and the simply supported $\left[\left( \pm 45 / 0_{2}\right)_{\mathrm{m}}\right]_{\mathrm{s}}$ laminates require the least amount of heating.

The results shown in Fig. 13 for the fully restrained plates indicate that the critical temperature change $\frac{12 b^{2}}{\pi^{2} t^{2}} \tilde{\Theta}_{0}^{\text {cr }}$ for the $[ \pm \theta]_{s}$ and $\left[( \pm \theta)_{m}\right]_{s}(m>5)$ laminates is highly dependent on the fiber angle $\theta$. In particular, the results for the clamped and simply supported $[ \pm \theta]_{s}$ and $\left[( \pm \theta)_{m}\right]_{s}(m>5)$ laminates show substantial increase in the critical temperature change with increasing $\theta$ for values up to approximately $55 \mathrm{deg}$ and $62 \mathrm{deg}$, respectively, followed by a significant decrease. In addition, the $\left[( \pm \theta)_{m}\right]_{s}(m>5)$ laminates require more heating to cause buckling than the corresponding $[ \pm \theta]_{\mathrm{s}}$ laminates. Overall, the plates with clamped edges are much more buckling resistant than the corresponding plates with simply supported edges, as expected.

The data presented in Figs. 12 and 13 are given in a compact form and are applicable to an infinite range of plate width-to-thickness ratios, $b / t$. Once the number of laminate plies is selected, the critical temperature change $\tilde{\Theta}_{0}^{\text {cr }}$ can be found as a function of the plate width $\mathrm{b}$ for a given laminate family. However, it is important to keep in mind the limitations of classical laminated-plate bending theory as the plate width-to-thickness ratio $b / t$ becomes smaller than a value of approximately 20 . Results of this type are useful in structural design and are illustrated for the fully restrained $\left[( \pm 45 / 0 / 90)_{m}\right]_{s}$ quasiisotropic laminates in Fig. 13 of Ref. 7.

The results in Figs. 14-17 indicate a general, common trend of a reduction in the critical-temperaturechange ratio $\frac{\Theta \delta_{i}^{r}}{\bar{\Theta}_{0}^{c_{1}}\left(1+C_{1}\right)}$ with increases in the nondimensional compliance ratio $\left(1+\mathrm{C}_{1}\right) /\left(1+\mathrm{C}_{2}\right)$, for all the laminates considered and both sets of flexural boundary conditions. This reduction in $\left(1+\mathrm{C}_{1}\right) /\left(1+\mathrm{C}_{2}\right)$ corresponds to a reduction in the thermally induced transverse compression load and hence the load factor $\mathrm{L}_{2}$. For many of the laminate configurations shown in the figures, the critical-temperature-change ratios are coincident with points of the gray curves that are defined by Eq. (91). In Fig. 14, the solid black curve shown is on top of the gray curve defined by Eq. (91). This coincidence indicates that an infinitely long laminate that is either fully restrained or elastically restrained exhibits a wide-column buckling mode for both restraint cases. In contrast, the curves shown in Figs. 14-17 that are not coincident with the solid gray curve, which are mostly for clamped plates, indicate the absence of a wide-column buckling mode. In addition, the effect of varying the number of plies in the $\left[( \pm 45 / 0 / 90)_{\mathrm{m}}\right]_{\mathrm{s}},\left[\left( \pm 45 / \mathrm{O}_{2}\right)_{\mathrm{m}}\right]_{\mathrm{s}}$, and $\left[\left( \pm 45 / 90_{2}\right)_{\mathrm{m}}\right]_{\mathrm{s}}$ laminates varies significantly with the laminate type. For plates with finite length, these trends are expected to change dramatically because moderate-aspect-ratio, finite-length plates do not exhibit wide-column buckling modes.

\section{Generic Results and Examples}

In Ref. 7, generic results are presented that enable one to obtain critical temperatures for a wide range of laminate configurations that are subjected to fully re- 
strained thermal expansion or contraction by using known buckling-behavior results for anisotropic plates subjected to combined mechanical loads. The work presented in Ref. 7 is based on the simplicity of equations like Eq. (49) and consists of data that define fundamental parameters for a wide variety of selected laminate constructions. Specifically, figures that show the stiffnessweighted laminate thermal-expansion parameters $\hat{\alpha}_{1}$, $\alpha_{2}$, and $\alpha_{3}$; the flexural orthotropy parameter $\beta$; and the flexural anisotropy parameters $\gamma$ and $\delta$ as a function of material system and laminate stacking sequence are presented in Ref. 7. In addition, figures that show the critical value of the loading parameter $\tilde{\mathrm{p}}_{\mathrm{cr}}$, or buckling interaction curves, as a function of flexural boundary conditions, $\beta, \gamma, \delta, \mathrm{L}_{1}, \mathrm{~L}_{2}$, and $\mathrm{L}_{3}$ are also presented for a broad range of parameters and combined mechanical loading conditions. Results of this type are significant because they illustrate the key aspects of the behavior and show overall trends and sensitivity of the behavior to changes in the parameters. Moreover, these results enable one to apply knowledge of, and experience with, the behavior of anisotropic plates subjected to mechanical loads to the problem of restrained thermal expansion or contraction.

The present study builds upon the work in Ref. 7 by presenting generic data in this section that can be used with the results in Ref. 7 to determine the effect of an elastic restraining medium, like the one shown in Fig. 3, on the thermal buckling behavior for a very wide range of laminate constructions and restraint configurations. Specifically, results are presented that show the effects of material systems and laminate stacking sequence on the fundamental parameters that are used to determine the thermally induced mechanical loads. Moreover, corresponding values of the fundamental nondimensional parameters $\beta, \gamma$, and $\delta$ that are required to use the generic mechanical-buckling-behavior results that appear in Ref. 7 are also presented. In addition, the procedure used to determine the critical temperature change by using the generic results is discussed.

\section{Values of the Fundamental Parameters}

Values of the fundamental parameters that are used herein are presented in this section for transversely stiff $\left[\left( \pm 45 / 90_{2}\right)_{\mathrm{m}}\right]_{\mathrm{s}}$ laminates, $\left[( \pm 45 / 0 / 90)_{\mathrm{m}}\right]_{\mathrm{s}}$ quasi-isotropic laminates, axially stiff $\left[\left( \pm 45 / 0_{2}\right)_{m}\right]_{s}$ laminates, and $\left[( \pm \theta)_{m}\right]_{s}$ angle-ply laminates. Nine different contemporary material systems are used. These material systems include boron-aluminum, S-glass-epoxy, a typical boron-epoxy, AS4/3501-6 graphite-epoxy, AS4/3502 graphite-epoxy, IM7/5260 graphite-bismaleimide, Kevlar 49-epoxy, IM7/PETI-5, and P-100/3502 pitch-epoxy materials. The mechanical properties of these material systems are presented in Table 1 and the nominal ply thickness is 0.005 in.

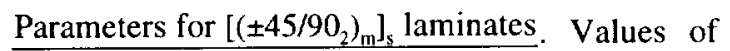
$\left(1+C_{1}\right)\left(1+T_{1}\right)$ and $\left(1+C_{1}\right) /\left(1+C_{2}\right)$ for $\left[\left( \pm 45 / 90_{2}\right)_{m}\right]_{s}$ laminates are presented in Figs. 18 and 19, respectively, as a function of the relative-compliance ratio $R_{1}$ for the nine material systems. The graph in Fig. $19 \mathrm{~b}$ is an enlargement of the portion of the graph in Fig. 19a that contains the closely grouped curves. The results in these figures are for the special case where the relative-compliance ratios are equal and the ratios of the coefficients of thermal expansion are equal $\left(R_{1}=R_{2}\right.$ and $\left.T_{1}=T_{2}\right)$, and are independent of the number of laminate plies. The results show a wide variation in $\left(1+C_{1}\right)\left(1+T_{1}\right)$ and $(1+$ $\left.\mathrm{C}_{1}\right) /\left(1+\mathrm{C}_{2}\right)$ with material system. Moreover, $\left(1+\mathrm{C}_{1}\right)(1$ $\left.+T_{1}\right)$ is positive for all the material systems, and the curves shown in Fig. 18 increase monotonically with increases in the relative-compliance ratio $R_{1}$, except the curve for the $\mathrm{P}-100 / 3502$ pitch-epoxy material. Similarly, $\left(1+C_{1}\right) /\left(1+C_{2}\right)$ is positive for all the material systems and the curves shown in Figs. 19a and $19 \mathrm{~b}$ decrease monotonically with increases in the relative-compliance ratio $R_{1}$, except the curve for the Kevlar 49-epoxy material. In addition, the magnitudes of $\left(1+C_{1}\right) /\left(1+C_{2}\right)$ for the Kevlar 49-epoxy material are much greater than those for the other eight materials. The curves for the boron-aluminum, S-glass-epoxy, and boron-epoxy materials exhibit values of $\left(1+C_{1}\right) /\left(1+C_{2}\right)$ that are nearly equal to unity, which indicates a relatively small contribution of the elastic restraining medium to the thermally induced transverse load ( $y$-coordinate direction shown in Fig. 2b).

Values of $\hat{\alpha}_{1}$ and $\hat{\alpha}_{2} / \hat{\alpha}_{1}$ for $\left[\left( \pm 45 / 90_{2}\right)_{m}\right]_{s}$ laminates are presented in Figs. 20 and 21 , respectively, as a function of the number of laminate plies for the nine material systems. The graph in Fig. $21 \mathrm{~b}$ is an enlargement of the portion of the graph in Fig. 21a that contains the closely grouped curves. The results show a wide variation in $\alpha_{1}$ with material system, a relatively benign variation with the number of plies, and that $\alpha_{1}$ is positive for all the material systems. However, $\hat{\alpha}_{1}$ is nearly zero valued for the P-100/3502 pitch-epoxy material. Likewise, the results in Figs. $21 \mathrm{a}$ and $21 \mathrm{~b}$ show a substantial variation in $\alpha_{2} / \alpha_{1}$ with material system, and that $\alpha_{2} / \alpha_{1}$ is positive for all the material systems except the P-100/ 3502 pitch-epoxy and Kevlar 49-epoxy materials. Specifically, the values for the Kevlar 49-epoxy material are slightly negative and nearly equal to zero and those for the P-100/3502 pitch-epoxy material are negative and have much larger magnitudes than the corresponding 
values for the other materials.

The results in Figs. 18 and 20 indicate that the values of $\frac{Q_{1}}{\left(1+C_{1}\right)\left(1+T_{1}\right)}$ are positive for the $\left[\left( \pm 45 / 90_{2}\right)_{m}\right]_{s}$ laminates, for all nine material systems, which means that $\alpha_{1} /\left(1+C_{1}\right)>0$ as long as $\left(1+T_{1}\right)>0$. Similarly, the results in Figs. 19 and 21 indicate that the values of $\left(\frac{1+C_{1}}{1+C_{2}}\right) \frac{\alpha_{2}}{\alpha_{1}}$ are positive for all the materials except the $P$ 100/3502 pitch-epoxy and Kevlar 49-epoxy materials. Thus, for the majority of the materials, the laminates are loaded by biaxial compression when uniformly heated and by biaxial tension when uniformly cooled, and as a result can buckle only for uniform heating as long as (1 $\left.+T_{1}\right)>0$ (see case 1). For the Kevlar 49-epoxy and P$100 / 3502$ pitch-epoxy materials, the laminates are loaded by axial compression and transverse tension when uniformly heated and by oppositely directed loads when uniformly cooled, as long as $\left(1+T_{1}\right)>0$. For this situation, the laminates can buckle for uniform heating (case 1) or uniform cooling (case 2). Overall, Figs. 18-21 show that there is a wide range of possibilities available for tailoring the thermal buckling characteristics of a laminate family and restraining medium.

The remaining parameters needed for a buckling analysis of the long transversely stiff $\left[\left( \pm 45 / 90_{2}\right)_{\mathrm{m}}\right]_{\mathrm{s}}$ laminates are the the nondimensional flexural orthotropy parameter $\beta$ and the flexural anisotropy parameters $\gamma$ and $\delta$. Values for these parameters are presented in Figs. 2224 as a function of the number of laminate plies, for the nine material systems. The results in Fig. 22 show a series of monotonically decreasing curves that each approach a constant value of $\beta$ from above as the number of plies increases. Similarly, the results in Figs. 23 and 24 show a series of monotonically decreasing curves that approach negligible values for $\gamma$ and $\delta$, respectively, from above as the number of plies increases. The largest and smallest values of $\beta, \gamma$ and $\delta$ are exhibited by the laminates made of the P-100/3502 pitch-epoxy and boron-aluminum materials, respectively.

Parameters for $\left[( \pm 45 / 0 / 90)_{m}\right]_{s}$ laminates. Values of

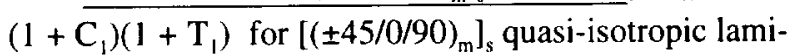
nates are presented in Fig. 25 as a function of the relative-compliance ratio $R_{1}$ for the nine material systems. The results in this figure are also for the special case where the relative-compliance ratios are equal and the ratios of the coefficients of thermal expansion are equal $\left(\mathbf{R}_{1}\right.$ $=R_{2}$ and $T_{1}=T_{2}$ ), and are independent of the number of laminate plies. Unlike the results for the $\left[\left( \pm 45 / 90_{2}\right)_{\mathrm{m}}\right]_{\mathrm{s}}$ laminates, the results in Fig. 25 show a relatively small variation in $\left(1+C_{1}\right)\left(1+T_{1}\right)$ with material system for the quasi-isotropic laminates. Moreover, $\left(1+C_{1}\right)\left(1+T_{1}\right)$ is positive for all the material systems, and the curves shown in Fig. 25 increase monotonically with increases in the relative-compliance ratio $R_{1}$, with no exceptions. Values for $\left(1+C_{1}\right) /\left(1+C_{2}\right)$ were determined to be equal to unity, for all nine material systems and for the values of the relative-compliance ratio $R_{1}$ shown in Fig. 25 . This result indicates that there is no contribution of the elastic restraining medium to the thermally induced transverse load ( $y$-coordinate direction shown in Fig. 2b).

Values of $\hat{\alpha}_{1}$ and $\hat{\alpha}_{2} / \hat{\alpha}_{1}$ for $\left[( \pm 45 / 0 / 90)_{\mathrm{m}}\right]_{\mathrm{s}}$ quasiisotropic laminates are presented for the nine material systems in Figs. 14 and 15, respectively, in Ref. 7. The results also show a wide variation in $\hat{\alpha}_{1}$ with material system for the quasi-isotropic laminates. Moreover, $\hat{\alpha}_{1}$ is positive for all the material systems except the P-100/ 3502 pitch-epoxy material. Similarly, $\hat{\alpha}_{2} / \alpha_{1}$ is positive for all the material systems.

The results in Fig. 14, in Ref. 7, and Fig. 25 herein indicate that the values of $\frac{\alpha_{1}}{\left(1+C_{1}\right)\left(1+T_{1}\right)}$ are positive for the $\left[( \pm 45 / 0 / 90)_{m}\right]_{s}$ quasi-isotropic laminates, for all nine materials, except the P-100/3502 pitch-epoxy material Thus, $\quad \alpha_{1} /\left(1+C_{1}\right)>0$ for all materials but the P-100/ 3502 pitch-epoxy material as long as $\left(1+T_{1}\right)>0$. Similarly, the results in Fig. 15, in Ref. 7, and the result $(1+$ $\left.C_{1}\right) /\left(1+C_{2}\right)=1$ indicate that the values of $\left(\frac{1+C_{1}}{1+C_{2}}\right) \frac{\alpha_{2}}{\alpha_{1}}$ are positive for all the materials. Thus, for all materials except the P-100/3502 pitch-epoxy material, the $[( \pm 45 / 0 /$ $\left.90)_{\mathrm{m}}\right]_{\mathrm{s}}$ quasi-isotropic laminates are loaded by biaxial compression when uniformly heated and by biaxial tension when uniformly cooled, and as a result can buckle only for uniform heating as long as $\left(1+T_{1}\right)>0$ (see case 1). For the P-100/3502 pitch-epoxy material, the laminates are loaded by biaxial tension when uniformly heated and by biaxial compression when uniformly cooled. These laminates must be cooled to buckle (case 1).

Values of the nondimensional orthotropy parameter $\beta$ and the nondimensional anisotropy parameters $\gamma$ and $\delta$ needed for a buckling analysis of the $[( \pm 45 / 0 /$ 90) $\mathrm{m}_{\mathrm{m}} \mathrm{s}_{\mathrm{s}}$ quasi-isotropic laminates made of the same 9 material systems have been presented graphically in Ref. 6 . The parameters are presented graphically in Ref. 6 as a function of the stacking-sequence number $m$, along with a discussion of their characteristics. The results in Ref. 6 for these parameters show a series of monotonically decreasing curves that approach $\beta=1, \gamma=0$, and $\delta=0$ from above as the number of plies increases. A homogeneous, isotropic material has values of $\beta=1, \gamma=0$, and $\delta=0$. Thus, the results for $\beta, \gamma$, and $\delta$ in Ref. 6 give, to 
some extent, a quantitative measure of quasi-isotropy.

Parameters for $\left[\left( \pm 45 / 0_{2}\right)_{m}\right]_{\mathrm{s}}$ laminates. Values of $\left(1+C_{1}\right)\left(1+T_{1}\right)$ and $\left(1+C_{1}\right) /\left(1+C_{2}\right)$ for axially stiff $\left[\left( \pm 45 / 0_{2}\right)_{\mathrm{m}}\right]_{\mathrm{s}}$ laminates are presented in Figs. 26 and 27 , respectively, as a function of the relative-compliance ratio $R_{1}$ for the nine material systems. The results in these figures are also for the special case where the relativecompliance ratios are equal and the ratios of the coefficients of thermal expansion are equal $\left(R_{1}=R_{2}\right.$ and $T_{1}=$ $\mathrm{T}_{2}$ ). Moreover, the results are independent of the number of laminate plies. Like for the $\left[\left( \pm 45 / 90_{2}\right)_{\mathrm{m}}\right]_{\mathrm{s}}$ laminates, the results in Figs. 26 and 27 for the $\left[\left( \pm 45 / 0_{2}\right)_{\mathrm{m}}\right]_{\mathrm{s}}$ laminates show a wide variation in $\left(1+C_{1}\right)\left(1+T_{1}\right)$ and $(1+$ $\left.C_{1}\right) /\left(1+C_{2}\right)$ with material system. Moreover, $\left(1+C_{1}\right)(1$ $\left.+T_{1}\right)$ is positive for all the material systems, and the curves shown in Fig. 26 increase monotonically with increases in the relative-compliance ratio $R_{1}$, except the curve for the Kevlar 49-epoxy material. Similarly, $(1+$ $\left.C_{1}\right) /\left(1+C_{2}\right)$ is positive for all the material systems and the curves shown in Fig. 27 increase monotonically with increases in the relative-compliance ratio $R_{1}$, except the curve for the Kevlar 49-epoxy material. In addition, the magnitudes of $\left(1+C_{1}\right) /\left(1+C_{2}\right)$ for the Kevlar 49-epoxy material are generally much smaller than those for the other eight materials. The trends exhibited by the curves in Fig. 27 for the $\left[\left( \pm 45 / 0_{2}\right)_{\mathrm{m}}\right]_{\mathrm{s}}$ laminates are generally opposite of the trends exhibited in Figs. 19a and $19 \mathrm{~b}$ for the corresponding $\left[\left( \pm 45 / 90_{2}\right)_{\mathrm{m}}\right]_{\mathrm{s}}$ laminates. The curves shown in Fig. 27 for the boron-aluminum, S-glass-epoxy, and boron-epoxy materials exhibit values of $(1+$ $\left.C_{1}\right) /\left(1+C_{2}\right)$ that are nearly equal to unity, which indicates a relatively small contribution of the elastic restraining medium to the thermally induced transverse load (y-coordinate direction shown in Fig. 2b).

Values of $\alpha_{1}$ and $\alpha_{2} / \alpha_{1}$ for $\left[\left( \pm 45 / 0_{2}\right)_{\mathrm{m}}\right]_{\mathrm{s}}$ laminates are presented in Figs. 28 and 29 , respectively, as a function of the number of laminate plies for the nine material systems. The graph in Fig. $29 \mathrm{~b}$ is an enlargement of the portion of the graph in Fig. 29a that contains the closely grouped curves. The results for these axially stiff laminates also show a wide variation in $\alpha_{1}$ with material system, and a generally benign variation with the number of plies, and that $\alpha_{1}$ is positive for all the materials except the P-100/3502 pitch-epoxy and Kevlar 49-epoxy materials. Moreover, $\alpha_{1}$ is nearly zero valued for the Kevlar 49-epoxy material. Likewise, the results in Figs. 29a and $29 \mathrm{~b}$ show a substantial variation in $\hat{\alpha}_{2} / \hat{\alpha}_{1}$ with material system, and that $\hat{\alpha}_{2} / \hat{\alpha}_{1}$ is positive for all the material systems except the P-100/3502 pitch-epoxy and Kevlar 49-epoxy materials. Specifically, the values for the $P$ -
100/3502 pitch-epoxy material are slightly negative and nearly equal to zero and those for the Kevlar 49-epoxy material are negative and have much larger magnitudes than the corresponding values for the other materials.

The results in Figs. 26 and 28 indicate that the values of $\frac{\alpha_{1}}{\left(1+C_{1}\right)\left(1+T_{1}\right)}$ are positive for the $\left[\left( \pm 45 / 0_{2}\right)_{m}\right]_{s}$ laminates, for all material systems, except the P-100/3502 pitch-epoxy and Kevlar 49-epoxy materials, which means that $\alpha_{1} /\left(1+C_{1}\right)>0$ as long as $\left(1+T_{1}\right)>0$. Similarly, the results in Figs. 27 and 29 indicate that the values of $\left(\frac{1+C_{1}}{1+C_{2}}\right) \frac{\alpha_{2}}{\alpha_{1}}$ are also positive for all the materials except the P-100/3502 pitch-epoxy and Kevlar 49-epoxy materials. Thus, for the majority of the materials, the laminates are loaded by biaxial compression when uniformly heated and by biaxial tension when uniformly cooled, and as a result can buckle only for uniform heating as long as $\left(1+T_{1}\right)>0$ (case 1$)$. For the $\mathrm{P}-100 / 3502$ pitch-epoxy and the Kevlar 49-epoxy materials, the laminates are loaded by axial tension and transverse compression when uniformly heated and by axial compression and transverse tension when uniformly cooled. For this situation, the laminates can buckle for uniform heating (case 2) or uniform cooling (case 1) as long as $\left(1+T_{1}\right)>0$.

The remaining parameters needed for a buckling analysis of the long, axially stiff $\left[\left( \pm 45 / 0_{2}\right)_{\mathrm{m}}\right]_{\mathrm{s}}$ laminates are the the nondimensional flexural orthotropy parameter $\beta$ and the flexural anisotropy parameters $\gamma$ and $\delta$. The values of $\beta$ for the $\left[\left( \pm 45 / 0_{2}\right)_{m}\right]_{5}$ laminates made of the nine material systems are identical to the corresponding values for the $\left[\left( \pm 45 / 90_{2}\right)_{\mathrm{m}}\right]_{\mathrm{s}}$ laminates that are shown in Fig. 22. Moreover, the values of $\gamma$ for the $\left[\left( \pm 45 / 0_{2}\right)_{m}\right]_{\mathrm{s}}$ laminates are identical to the corresponding values of $\delta$ that are shown in Fig. 24 for the $\left[\left( \pm 45 / 90_{2}\right)_{\mathrm{m}}\right]_{\mathrm{s}}$ laminates. Furthermore, the values of $\delta$ for the $\left[\left( \pm 45 / 0_{2}\right)_{n}\right]_{s}$ laminates are identical to the corresponding values of $\gamma$ that are shown in Fig. 23 for the $\left[\left( \pm 45 / 90_{2}\right)_{m}\right]_{\mathrm{s}}$ laminates.

Parameters for $\left[( \pm \theta)_{m}\right]_{s}$ laminates. Values of the parameters $\frac{\alpha_{1}}{\left(1+C_{1}\right)\left(1+T_{1}\right)}$ and $\frac{\alpha_{2}}{\left(1+C_{2}\right)\left(1+T_{1}\right)}$ for $\left[( \pm \theta)_{m}\right]_{s}$ balanced, angle-ply laminates composed of the 9 material systems are presented in Figs. 30-35. The results in Figs. 30 and 31,32 and 33 , and 34 and 35 correspond to values of the relative-compliance ratios $R_{1}=R_{2}=0,0.2$, and 0.5 , respectively, and are independent of the number of laminate plies. For all the results in Figs. 30-35, the ratios of the coefficients of thermal expansion are equal $\left(T_{1}\right.$ $=\mathrm{T}_{2}$ ).

The results show an extremely wide variation in 
$\frac{a_{1}}{\left(1+C_{1}\right)\left(1+T_{1}\right)}$ and $\frac{a_{2}}{\left(1+C_{2}\right)\left(1+T_{1}\right)}$ with material system and with fiber angle $\theta$. The largest variations are exhibited by $\frac{\alpha_{2}}{\left(1+C_{2}\right)\left(1+T_{1}\right)}$, followed by $\frac{\alpha_{1}}{\left(1+C_{1}\right)\left(1+T_{1}\right)}$, for all values of the relative-compliance ratios considered. Moreover, $\frac{a_{1}}{\left(1+C_{1}\right)\left(1+T_{1}\right)}$ and $\frac{a_{2}}{\left(1+C_{2}\right)\left(1+T_{1}\right)}$ are generally positive for all the material systems except, for the most part, for the P-100/3502 pitch-epoxy and Kevlar 49-epoxy materials, which are negative for several values of the fiber angle $\theta$. Thus, depending on the material system and fiber angle, a laminate may be loaded by various combinations of tension and compression and may buckle when subjected to uniform heating, cooling, or both. Like the results for the transversely stiff $\left[\left( \pm 45 / 90_{2}\right)_{\mathrm{m}}\right]_{\mathrm{s}}$ laminates, the $[( \pm 45 /$ $\left.0 / 90)_{\mathrm{m}}\right]_{\mathrm{s}}$ quasi-isotropic laminates, and the axially stiff $\left[\left( \pm 45 / 0_{2}\right)_{\mathrm{m}}\right]_{\mathrm{s}}$ laminates, the results in Figs. 30-35 show that there is a very wide range of possibilities available for tailoring the thermal buckling characteristics of a laminate family.

Values of the nondimensional orthotropy parameter $\beta$ and the nondimensional anisotropy parameters $\gamma$ and $\delta$ for the $\left[( \pm \theta)_{m}\right]_{s}$ laminates made of the same 9 material systems have also been presented graphically in Ref. 6. The values of $\beta$ in Ref. 6 for the $\left[( \pm \theta)_{\mathrm{m}}\right]_{\mathrm{s}}$ laminates are independent of the stacking-sequence number $\mathrm{m}$. The results in Ref. 6 show a series of curves for $\beta$ that vary dramatically with the fiber angle $\theta$ and the material system. The largest values of, and greatest variations in, $\beta$ are generally exhibited by the laminates made of the P-100/3502 pitch-epoxy material. In contrast, the smallest values of, and least variations in, $\beta$ are generally exhibited by the laminates made of the boron-aluminum material. The values of $\gamma$ and $\delta$ for the $\left[( \pm \theta)_{m}\right]_{s}$ laminates are strongly dependent on the stacking-sequence number $\mathrm{m}$. The results in Ref. 6 for the flexural anisotropy parameters $\gamma$ and $\delta$ are for $m=1$, which corresponds to the highest degree of flexural anisotropy for these laminates. The graphical results in Ref. 6 show a large effect of the fiber angle $\theta$ and the material system on the degree of flexural anisotropy for the $[ \pm \theta]_{s}$ laminates. Like the parameter $\beta$, the largest values of, and greatest variations in, $\gamma$ and $\delta$ are generally exhibited by the laminates made of the $\mathrm{P}-100 / 3502$ pitch-epoxy material, and the smallest values of, and least variations in, $\gamma$ and $\delta$ are generally exhibited by the laminates made of the boron-aluminum material. A comparison of results for $\left[( \pm \theta)_{6}\right]_{\mathrm{s}}$ and $[ \pm \theta]_{\mathrm{s}}$ laminates made of IM7/ 5260 graphite-bismaleimide material is presented in Ref. 7 (Fig. 21) that indicates that the flexural anisotropy of the $\left[( \pm \theta)_{6}\right]_{s}$ laminates is negligible compared to that of the corresponding $[ \pm \theta]_{\mathrm{s}}$ laminates. A similar trend is ex- pected for the corresponding laminates made from most of the other eight material systems.

\section{Buckling Coefficients and Critical Temperature Change}

In the present study, the approach that is used to compute critical temperature changes for symmetric laminates restrained by the apparatus shown in Fig. 3, in a generic setting, follows the scenarios designated herein as cases 1 through 4 . Essentially, one first selects a candidate laminate and computes the nondimensional parameters $\beta, \gamma$, and $\delta$ (see Eqs. (1)-(4)). Next, the load factor $\mathrm{L}_{2}$ that defines the thermally induced mechanical loads is calculated (e.g., by using Eqs. (47) or (50)) for a given restraint-medium configuration. This step involves the calculation of the stiffness-weighted laminate thermal-expansion parameters $\hat{\alpha}_{1}$ and $\hat{\alpha}_{2}$, and the calculation of the compliance parameters $\left(1+C_{1}\right)$ and $\left(1+C_{1}\right) /\left(1+C_{2}\right)$. The parameter $\left(1+C_{1}\right) /\left(1+C_{2}\right)$ represents the contribution of the elastic restraining medium to the thermally induced transverse load ( $y$-coordinate direction shown in Fig. 2b). Equivalently, the load factor $\mathrm{L}_{2}$ can be calculated from the appropriate values of $\alpha_{1} /\left(1+C_{1}\right)$ and $\alpha_{2} /\left(1+C_{2}\right)$. Then, the critical loading parameter is determined from figures such as Figs. 22-26 in Ref. 7, which give the critical (mechanical) loading parameter $\tilde{\mathrm{p}}_{\mathrm{cr}}$ as a function of $\beta, \gamma, \delta, \mathrm{L}_{2}$, and the flexural boundary conditions. Finally, the critical temperature change is calculated by using, for example, Eqs. (49) or (52).

\section{Examples}

To illustrate the use of the results presented previously herein with the generic results presented in Ref. 7, first consider a simply supported $\left[\left( \pm 45 / 90_{2}\right)_{8}\right]_{\mathrm{s}}$ laminate made of IM7/5260 graphite-bismaleimide material and restrained against thermal expansion or contraction by the apparatus depicted in Fig. 3. Moreover, consider a restraining medium that is define by the relative-compliance values $R_{1}=R_{2}=0.5$ and ratios of the coefficients of thermal expansion that are given by $T_{1}=T_{2}>-1$ and whose specific values will be defined later. For these values, the axial and transverse compliance of the restraining medium are half of the respective axial and transverse compliances of the plate (twice as stiff in each direction). From Figs. 22-24, one can estimate that $\beta \approx$ 1.3 and $\gamma=\delta \approx 0$. Effectively, this laminate is flexurally orthotropic. Similarly, from a laminate analysis code, or from Figs. 20 and 21 , one could get $\alpha_{1}=2.3 \times 10^{-6} / \mathrm{F}$, and $\alpha_{2} / \alpha_{1}=0.3$. In addition, Fig. 8 gives $\left(1+C_{1}\right)(1+$ $\left.\mathrm{T}_{1}\right)=1.6$ and Fig. 9 gives $\left(1+\mathrm{C}_{1}\right) /\left(1+\mathrm{C}_{2}\right)=0.6$. 
Combining these results gives $\hat{\alpha}_{1} /\left(1+C_{1}\right)=1.4\left(1+T_{1}\right)$ $\times 10^{-6} / \mathrm{F}$ and $\left(\frac{1+C_{1}}{1+C_{2}}\right) \frac{\alpha_{2}}{\alpha_{1}}=0.2$. For this laminate, the only destabilizing loads are biaxial compression loads that are obtained for uniform heating (case 1). For this case, $\mathrm{L}_{1}=1, \mathrm{~L}_{3}=0$, and $\mathrm{L}_{2}=0.2$ is obtained from Eq. (47). Note that $L_{2}=0.3$ for the same plate fully restrained against thermal expansion or contraction. Thus, the compliance of the boundary has substantially reduced the thermally induced transverse load. For $L_{1}=1$, $K_{x}=\tilde{p}_{c r}$. The value of $K_{x}$ is obtained from Fig. 23 in Ref. 7 by interpolating the results in the figure for $\beta=1$ and $\beta=1.5$. For $\beta=1$ and $L_{2}=0.2$, Fig. 23 in Ref. 7 gives $K_{x}=3.2$. Similarly, for $\beta=1.5$ and $L_{2}=0.2$, Fig. 23 gives $K_{x}=3.9$. Interpolating these values gives $K_{x}=3.6$ for $\beta=1.3$. Substituting $K_{x}=3.6$ for $\tilde{p}_{\mathrm{cr}}$ and $\alpha_{1} /\left(1+C_{1}\right)=1.4\left(1+T_{1}\right) \times 10^{-6} /{ }^{\circ} \mathrm{F}$ into Eq. (49) gives $\frac{12 b^{2}}{\pi^{2} t^{2}} \Theta_{0}^{\text {cr }}=\frac{2.6}{1+T_{1}} \times 10^{6}$ "F. Next, let the plate width be given by $b=32$ in. such that the plate width-to-thickness ratio is $b / t=100$. For this plate, $\Theta_{0}^{\mathrm{cr}} \approx \frac{214^{\prime \prime} F}{1+T_{1}}$. This result indicates that the critical temperature decreases for $T_{1}>$ 0 , which corresponds to the situation where the restraining media is expanding and providing additional compression loads on the laminate. For $T_{1}=0$, the relative thermal expansion or contraction of the restraining medium is negligible. For $-1<\mathrm{T}_{1}<0$, the restraining medium is contracting relative to the laminate expansion and the critical temperature increases. As $T_{1}$ approaches -1 , the expansion of the laminate is matched by expansion of the restraining medium and buckling cannot occur. Moreover, for values of $-1<T_{1}<0$, the possibility of elastic buckling may not exist. The corresponding critical temperature for the plate fully restrained against thermal expansion or contraction $\left(C_{1}=C_{2}=0\right)$ and with a thermally neutral restraining medium is approximately $112^{\circ} \mathrm{F}$.

Next, consider a simply supported $\left[ \pm 45 / 90_{2}\right]_{\mathrm{s}}$ flexurally anisotropic laminate made of IM7/5260 graphitebismaleimide material and also restrained against thermal expansion or contraction by the apparatus depicted in Fig. 3 with $R_{1}=R_{2}=0.5$. Likewise, the ratios of the coefficients of thermal expansion that are given by $T_{1}=$ $\mathrm{T}_{2}>-1$. From Figs. $22-24$, one can estimate that $\beta \approx 2.0$ and $\gamma=\delta \approx 0.2$. From a laminate analysis code, or from Figs. 20 and 21 , one could get $\alpha_{1}=2.4 \times 10^{-6} \rho \mathrm{F}$, and $\alpha_{2} / \alpha_{1}=0.5$. In addition, Fig. 8 gives $\left(1+\mathrm{C}_{1}\right)\left(1+\mathrm{T}_{1}\right)=$ 1.6 and Fig. 9 gives $\left(1+C_{1}\right) /\left(1+C_{2}\right)=0.6$. Combining these results gives $\alpha_{1} /\left(1+C_{1}\right)=1.5\left(1+T_{1}\right) \times 10^{-6} /$
"F and $\left(\frac{1+C_{1}}{1+C_{2}}\right) \frac{\alpha_{2}}{\alpha_{1}}=0.3$. For this laminate, the only destabilizing loads are again biaxial compression loads that are obtained for uniform heating (case 1). For this case, $L_{1}=1, L_{3}=0$, and $L_{2}=0.3$ is obtained from Eq. (47). Note once again that $L_{2}=0.5$ for the same plate fully restrained against thermal expansion or contraction. Thus, the compliance of the boundary has substantially reduced the thermally induced transverse load. Again, for $L_{1}=1, K_{x}=\tilde{p}_{c r}$. The value of $K_{x}$ is obtained directly from Fig. 30 in Ref. 7 and is given by $K_{x}=3.3$, which corresponds to a wide-column buckling mode. Substituting $\mathrm{K}_{\mathrm{x}}=3.3$ for $\tilde{\mathrm{p}}_{\mathrm{cr}}$ and $\alpha_{1} /\left(1+\mathrm{C}_{1}\right)=1.5\left(1+\mathrm{T}_{1}\right) \mathrm{x}$ $10^{-6} / \rho \mathrm{F}$ into Eq. (49) gives $\frac{12 \mathrm{~b}^{2}}{\pi^{2} \mathrm{t}^{2}} \Theta_{0}^{\mathrm{cr}}=\frac{2.2}{1+\mathrm{T}_{1}} \times 10^{6} \mathrm{~g} \mathrm{~F}$. Next, let the plate width be given by $b=32$ in. such that the plate width-to-thickness ratio is $b / t=100$. For this flexurally anisotropic plate, $\Theta_{01}^{\mathrm{cr}} \approx \frac{181^{\circ} \mathrm{F}}{1+\mathrm{T}_{1}}$. The corresponding critical temperature for the plate fully restrained against thermal expansion or contraction and with a thermally neutral restraining medium is approximately $66^{\circ} \mathrm{F}$.

\section{Concluding Remarks}

An analytical approach for synthesizing buckling results and behavior for long, balanced and unbalanced symmetric laminates that are subjected to uniform heating or cooling, and elastically restrained thermal expansion or contraction has been presented. A nondimensional buckling analysis for long flexurally anisotropic plates that are subjected to combined loads has been described and useful nondimensional parameters have been presented. In particular, stiffness-weighted thermal-expansion parameters and nondimensional compliance parameters have been presented that can be used to determine critical temperatures, for a wide range of laminate constructions and restraining media, in terms of physically intuitive, well-known mechanical-buckling coefficients. Moreover, the effects of membrane orthotropy and membrane anisotropy on the thermally induced prebuckling stress state have been determined for a general elastic restraining medium.

A large number of results have been presented herein for some common laminates that are intended, to some extent, to facilitate a structural designer's transition to the use of the generic buckling design curves that are included and discussed in the paper. Many of the results were previously unknown. In addition, several results have been presented that show the effect of laminate construction and restraining-media compliance on the buckling behavior, and several cases are presented that 
indicate when a laminate will buckle because of uniform cooling. Results of this type could be important in the design of vehicles that use liquid fuels or cruise at high altitudes. The use of generic buckling design curves that provide physical insight into the buckling problem of the present paper in addition to providing useful design data has also been discussed. In addition, examples have been presented that demonstrate the use of the generic design curves. Overall, the analysis approach and generic results that have been presented indentify the effects or characteristics of elastically restrained laminate thermal expansion or contraction, membrane orthotropy and anisotropy, and flexural orthotropy and anisotropy on laminated-plate buckling in a very general and unifying manner. Although the results are based on classical laminated-plate theory and have been demonstrated for infinitely long plates, the approach is applicable to more sophisticated plate theories that incorporate effects such as transverse-shear flexibility and can be used for finitelength plates in many cases.

\section{References}

'Nemeth, M. P., "Buckling Behavior of Long Symmetrically Laminated Plates Subjected to Combined Loadings," NASA TP 3195, May 1992.

${ }^{2}$ Nemeth, M. P., "Buckling Behavior of Long Symmetrically Laminated Plates Subjected to Compression, Shear, and Inplane Bending Loads," AIAA Journal, Vol. 30, No. 12, December 1992, pp. 2959-2965.

${ }^{3}$ Nemeth, M. P., "Buckling Behavior of Long Anistropic Plates Subjected to Combined Loads," NASA TP 3568, November 1995.
${ }^{4}$ Nemeth, M. P., "Buckling Behavior of Long Symmetrically Laminated Plates Subjected to Shear and Linearly Varying Axial Edge Loads," NASA TP 3659, July 1997.

${ }^{5}$ Nemeth, M. P., "Buckling Behavior of Long Anisotropic Plates Subjected to Restrained Thermal Expansion and Mechanical Loads," AIAA Paper N0. 99-1229, April 1999.

${ }^{6}$ Nemeth, M. P., "Buckling Behavior of Long Anisotropic Plates Subjected to Restrained Thermal Expansion and Mechanical Loads," Journal of Thermal Stresses, Vol. 23, 2000, pp. 873-916.

${ }^{7}$ Nemeth, M. P., "Buckling Behavior of Long Anisotropic Plates Subjected to Fully Restrained Thermal Expansion," AIAA Paper No. 2001-1330, April 2001.

${ }^{8}$ Nemeth, M. P., "Importance of Anisotropy on Buckling of Compression-Loaded Symmetric Composite Plates," AIAA Journal, Vol. 24, No. 11, November 1986 , pp. 1831-1835.

${ }^{9}$ Whitney, J. M., Structural Analysis of Laminated Anisotropic Plates, Technomic Publishing Co., Inc., Lancaster, Pennsylvania, 1987.

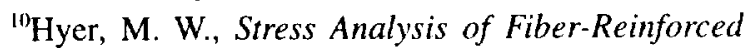
Composite Materials, WCB/McGraw Hill Publishing Co., Inc., Boston, 1998.

Table 1. Lamina Properties

\begin{tabular}{|c|c|c|c|c|c|c|c|c|c|}
\hline \multirow{2}{*}{$\begin{array}{c}\text { Lamina } \\
\text { property* }\end{array}$} & \multicolumn{9}{|c|}{ Material Systems } \\
\cline { 2 - 11 } & $\begin{array}{c}\text { Boron } \\
-\mathrm{Al}\end{array}$ & $\begin{array}{c}\text { S-glass- } \\
\text { epoxy }\end{array}$ & $\begin{array}{c}\text { Kevlar 49- } \\
\text { epoxy }\end{array}$ & $\begin{array}{c}\text { IM7/ } \\
5260\end{array}$ & $\begin{array}{c}\text { AS4/ } \\
3502\end{array}$ & $\begin{array}{c}\text { AS4/ } \\
3501-6\end{array}$ & $\begin{array}{c}\text { Boron- } \\
\text { epoxy }\end{array}$ & $\begin{array}{c}\text { IM7/ } \\
\text { PETI-5 }\end{array}$ & $\begin{array}{c}\text { P-100/ } \\
3502\end{array}$ \\
\hline $\mathrm{E}_{\mathrm{L}}, \mathrm{Msi}$ & 33 & 7.5 & 11.02 & 22.1 & 18.5 & 20.01 & 29.58 & 20.35 & 53.5 \\
\hline $\mathrm{E}_{\mathrm{T}}, \mathrm{Msi}$ & 21 & 1.7 & 0.8 & 1.457 & 1.64 & 1.30 & 2.68 & 1.16 & 0.73 \\
\hline $\mathrm{v}_{\mathrm{LT}}$ & 0.23 & 0.25 & 0.34 & 0.258 & 0.30 & 0.30 & 0.23 & 0.29 & 0.31 \\
\hline $\mathrm{G}_{\mathrm{LT}} \mathrm{Msi}$ & 7.0 & 0.80 & 0.33 & 0.860 & 0.87 & 1.03 & 0.81 & 0.61 & 0.76 \\
\hline$\alpha_{\mathrm{L}} \times 10^{6} \% \mathrm{~F}$ & 3.2 & 3.5 & -2.22 & 0.0125 & 0.25 & -0.167 & 3.38 & -0.14 & -0.64 \\
\hline$\alpha_{\mathrm{T}} \times 10^{6} / \mathrm{F}$ & 11.0 & 11.0 & 43.89 & 14.91 & 16.2 & 15.6 & 16.83 & 16.85 & 17.2 \\
\hline
\end{tabular}

* The symbols $\mathrm{L}$ and $\mathrm{T}$ denote the longitudinal fiber and transverse matrix directions of a specially orthotropic lamina, respectively. 


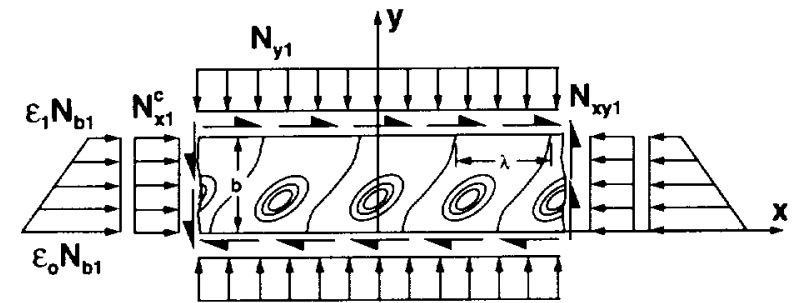

(a) Destabilizing loading system

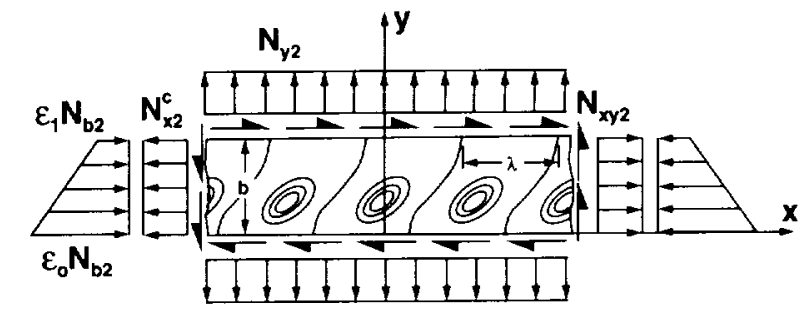

(b) Subcritical loading system

Fig. 1 Sign convention for positive-valued stress resultants.

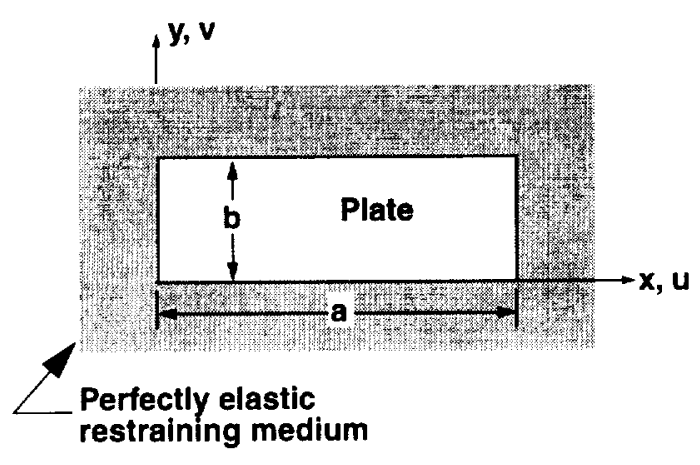

(a) General restraining medium

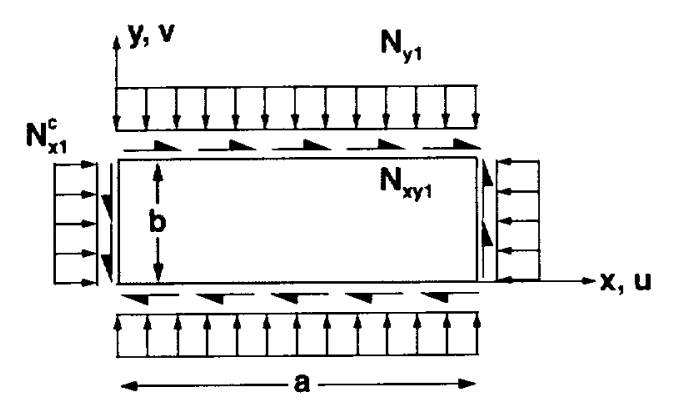

(b) Positive values for equivalent mechanical loads

Fig. 2 Mechanical loads in a plate elastically restrained against thermal expansion or contraction caused by uniform heating or cooling.

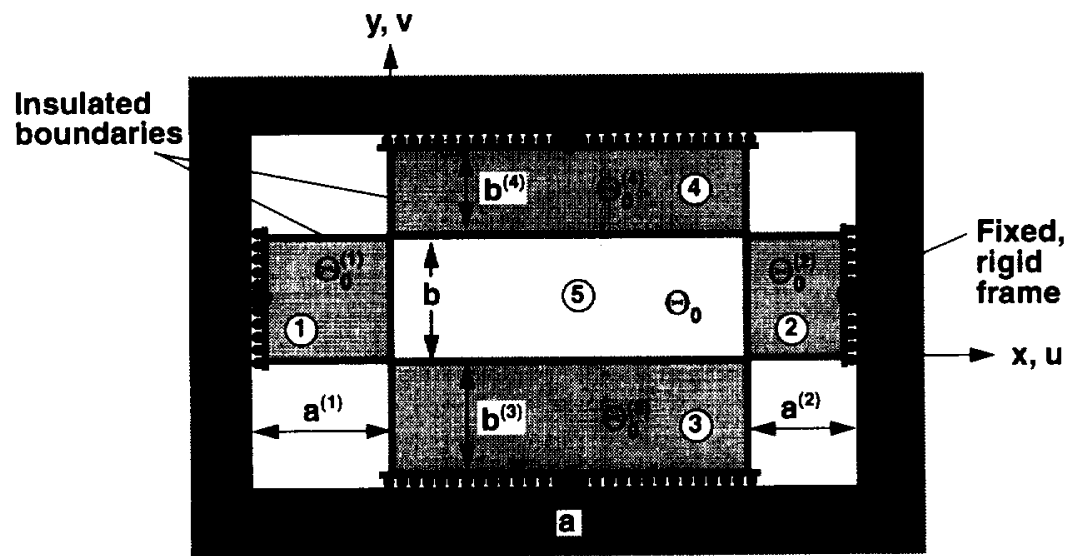

Fig. 3 Central plate elastically restrained by edge plates against thermal expansion or contraction caused by uniform heating or cooling in all plates. 


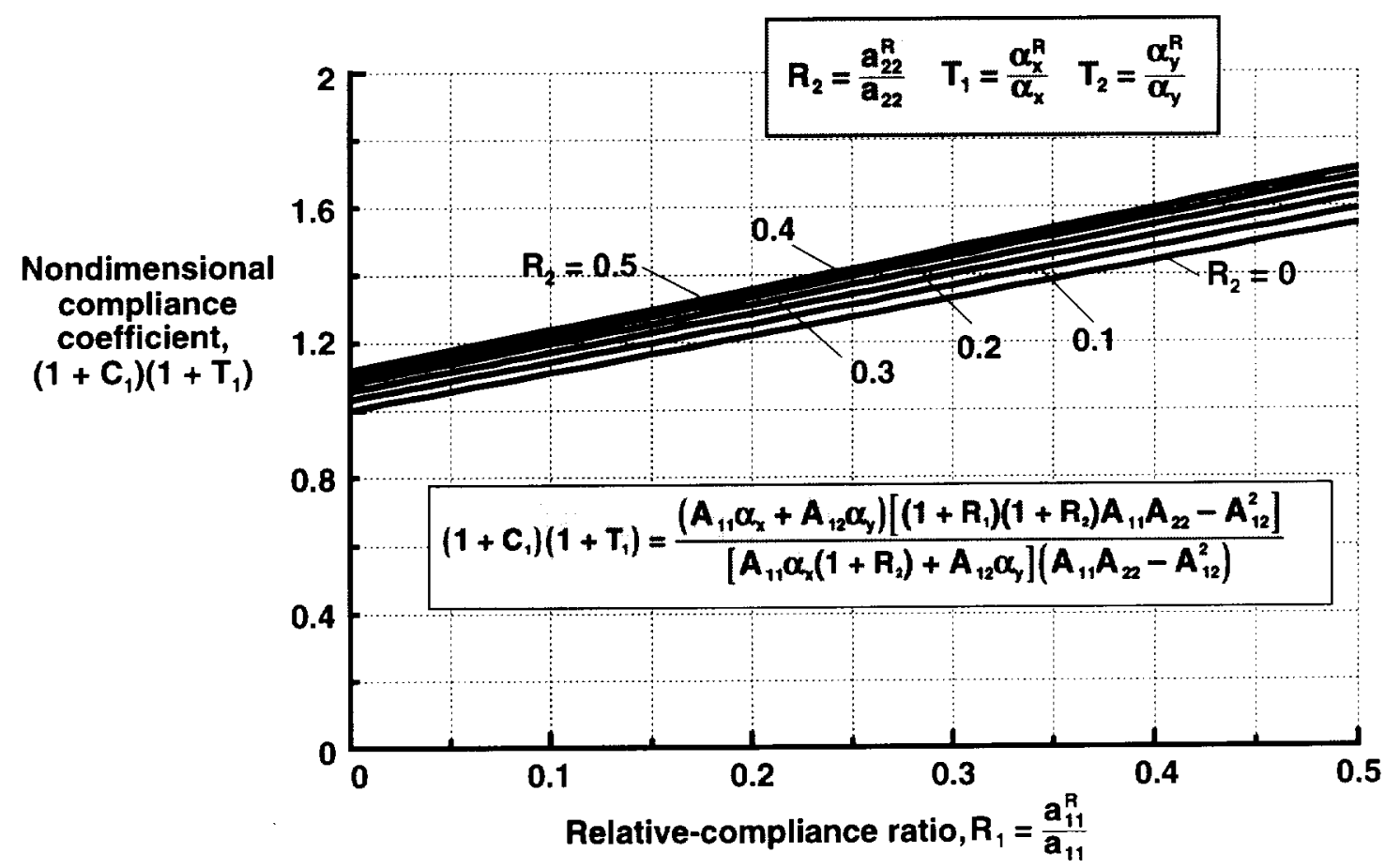

Fig. 4 Nondimensional compliance coefficient $\left(1+C_{1}\right)\left(1+T_{1}\right)$ for $\left[( \pm 45 / 0 / 90)_{m}\right]_{s}$ laminates made of IM7/5260 material and elastically restrained from thermal expansion or contraction by edge plates $\left(T_{1}=T_{2}\right.$, $\mathbf{m}=1,2, \ldots)$.

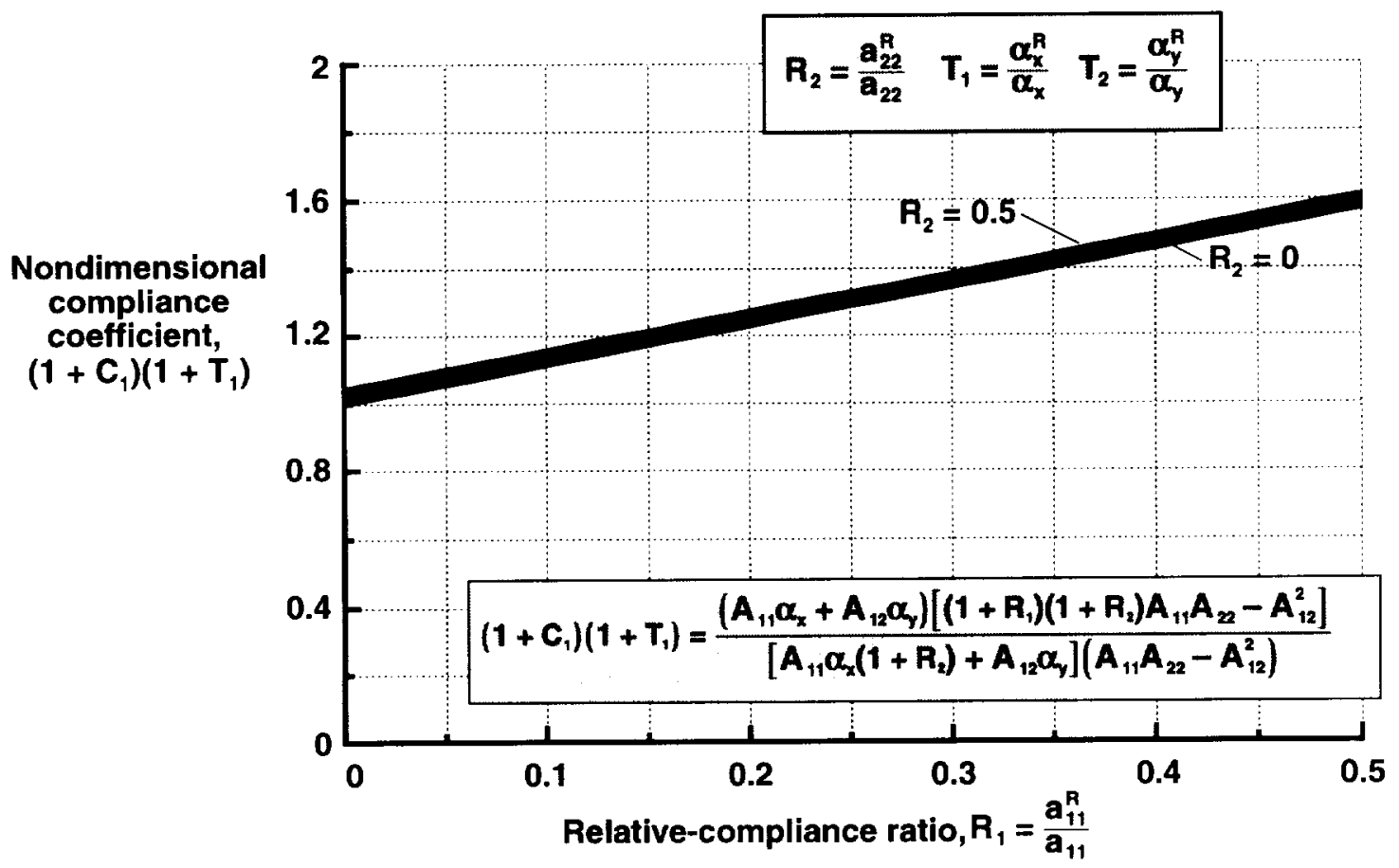

Fig. 5 Nondimensional compliance coefficient $\left(1+C_{1}\right)\left(1+T_{1}\right)$ for $\left[\left( \pm 45 / 90_{2}\right)_{m}\right]_{s}$ laminates made of IM7/5260 material and elastically restrained from thermal expansion or contraction by edge plates $\left(T_{1}=T_{2}, m=1,2, \ldots\right)$. 


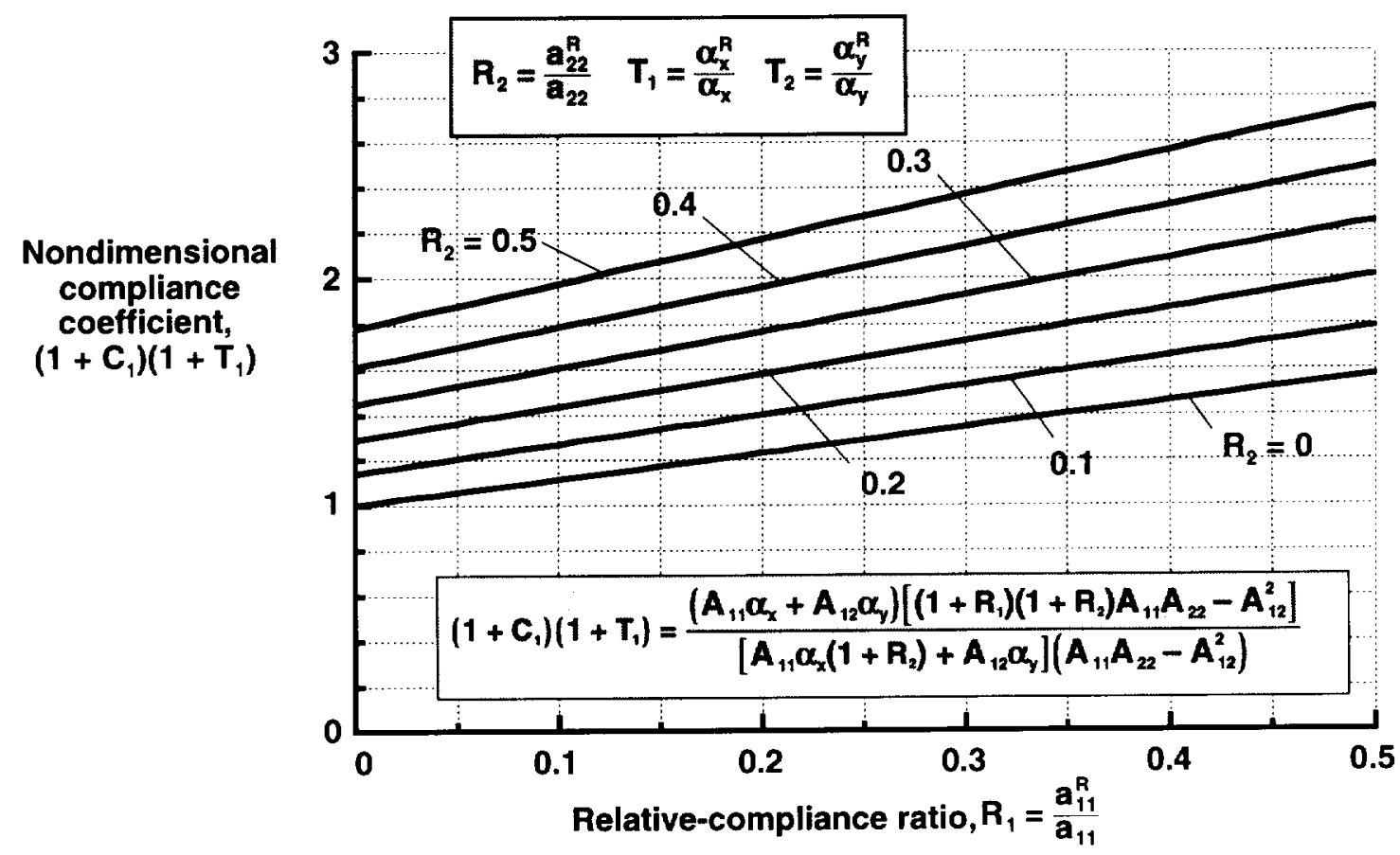

Fig. 6 Nondimensional compliance coefficient $\left(1+C_{1}\right)\left(1+T_{1}\right)$ for $\left[\left( \pm 45 / 0_{2}\right)_{m}\right]_{s}$ laminates made of IM7/5260 material and elastically restrained from thermal expansion or contraction by edge plates $\left(\mathbf{T}_{1}=\mathrm{T}_{2}, \mathrm{~m}=1,2, \ldots\right)$.

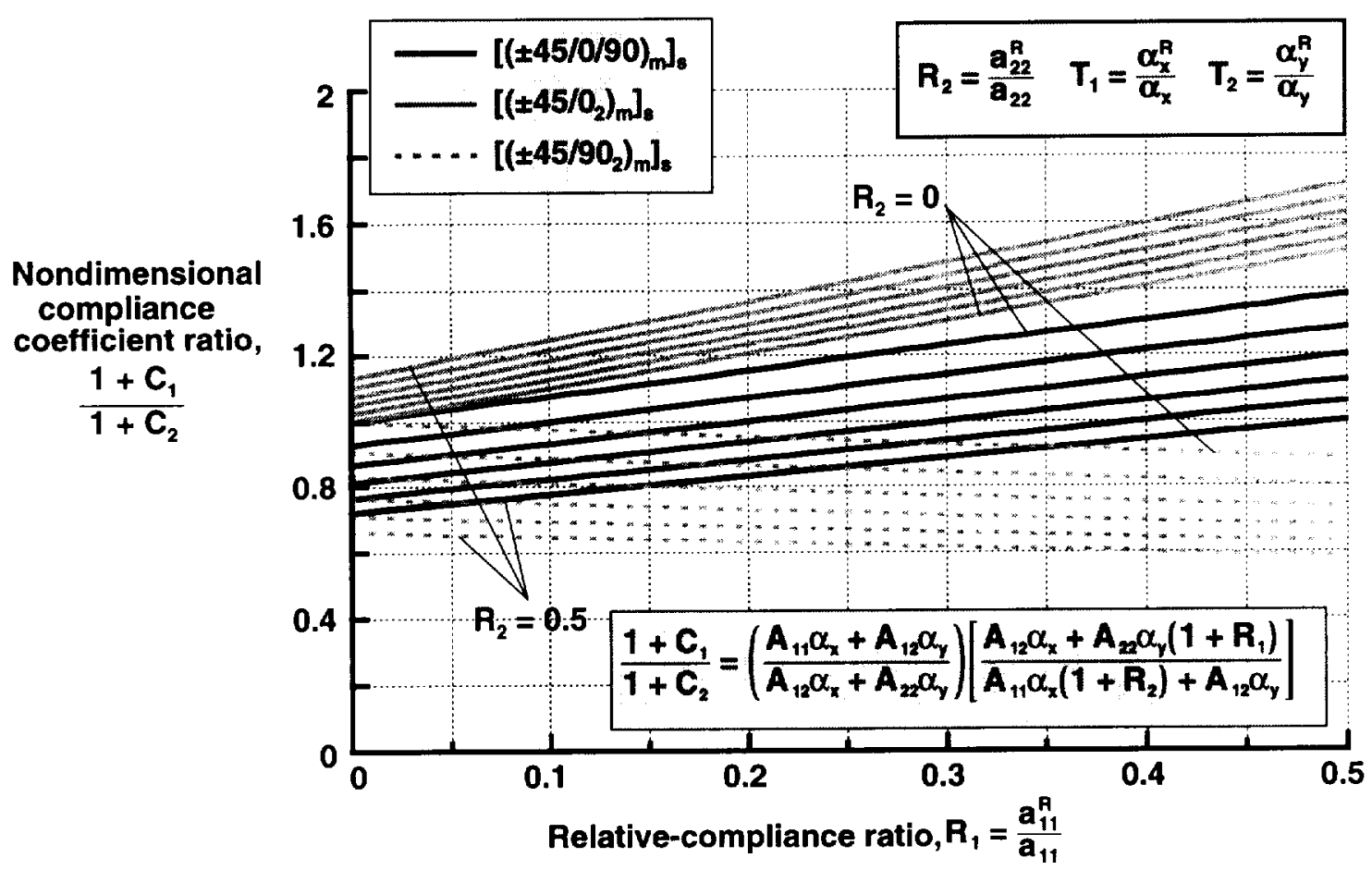

Fig. 7 Nondimensional compliance coefficient ratio $\left(1+C_{1}\right) /\left(1+C_{2}\right)$ for $\left[( \pm 45 / 0 / 90)_{m} l_{s},\left[\left( \pm 45 / 0_{2}\right)_{m}\right]_{s}\right.$, and $\left[( \pm 45 / 90,)_{\mathrm{m}}\right]_{\mathrm{s}}$ laminates made of $\mathrm{IM} 7 / 5260$ material and elastically restrained from thermal expansion or contraction by by edge plates $\left(T_{1}=T_{2}\right.$ and $\left.m=1,2, \ldots\right)$. 


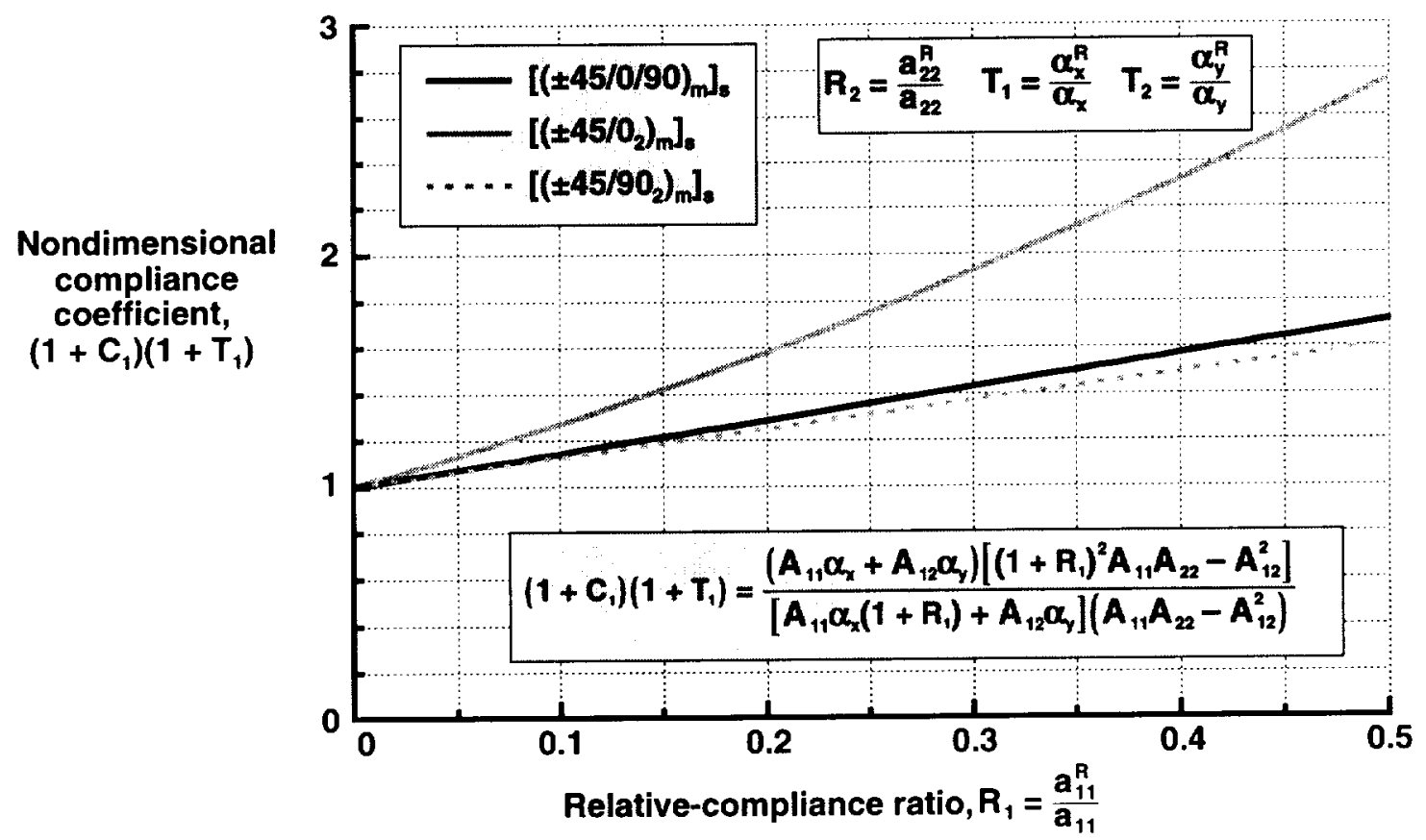

Fig. 8 Nondimensional compliance coefficient $\left(1+C_{1}\right)\left(1+T_{1}\right)$ for $\left[( \pm 45 / 0 / 90)_{m}\right]_{s},\left[\left( \pm 45 / 0_{2}\right)_{m}\right]_{s}$, and $\left[\left( \pm 45 / 90_{2}\right)_{m}\right]_{s}$ laminates made of IM7/5260 material and elastically restrained from thermal expansion or contraction by edge plates $\left(R_{2}=R_{1}, T_{1}=T_{2}\right.$, and $\left.m=1,2, \ldots\right)$.

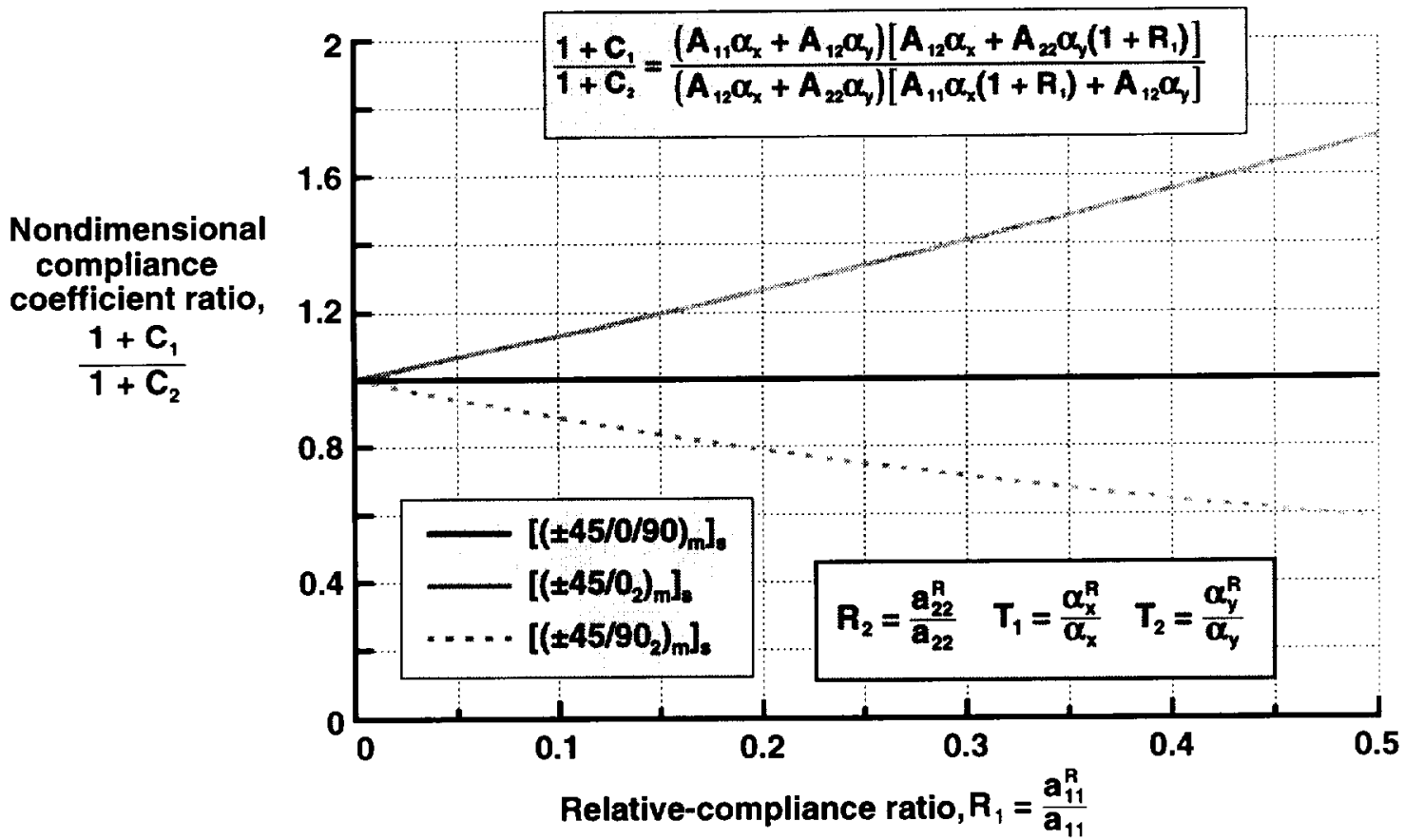

Fig. 9 Nondimensional compliance coefficient ratio $\left(1+C_{1}\right) /\left(1+C_{2}\right)$ for $\left[( \pm 45 / 0 / 90)_{m}\right]_{s},\left[\left( \pm 45 / 0_{2}\right)_{m}\right]_{s}$, and $\left[( \pm 45 / 90,)_{m}\right]_{s}$ laminates made of IM7/5260 material and elastically restrained from thermal expansion or contraction by edge plates $\left(R_{2}=R_{1}, T_{1}=T_{2}\right.$, and $\left.m=1,2, \ldots\right)$. 


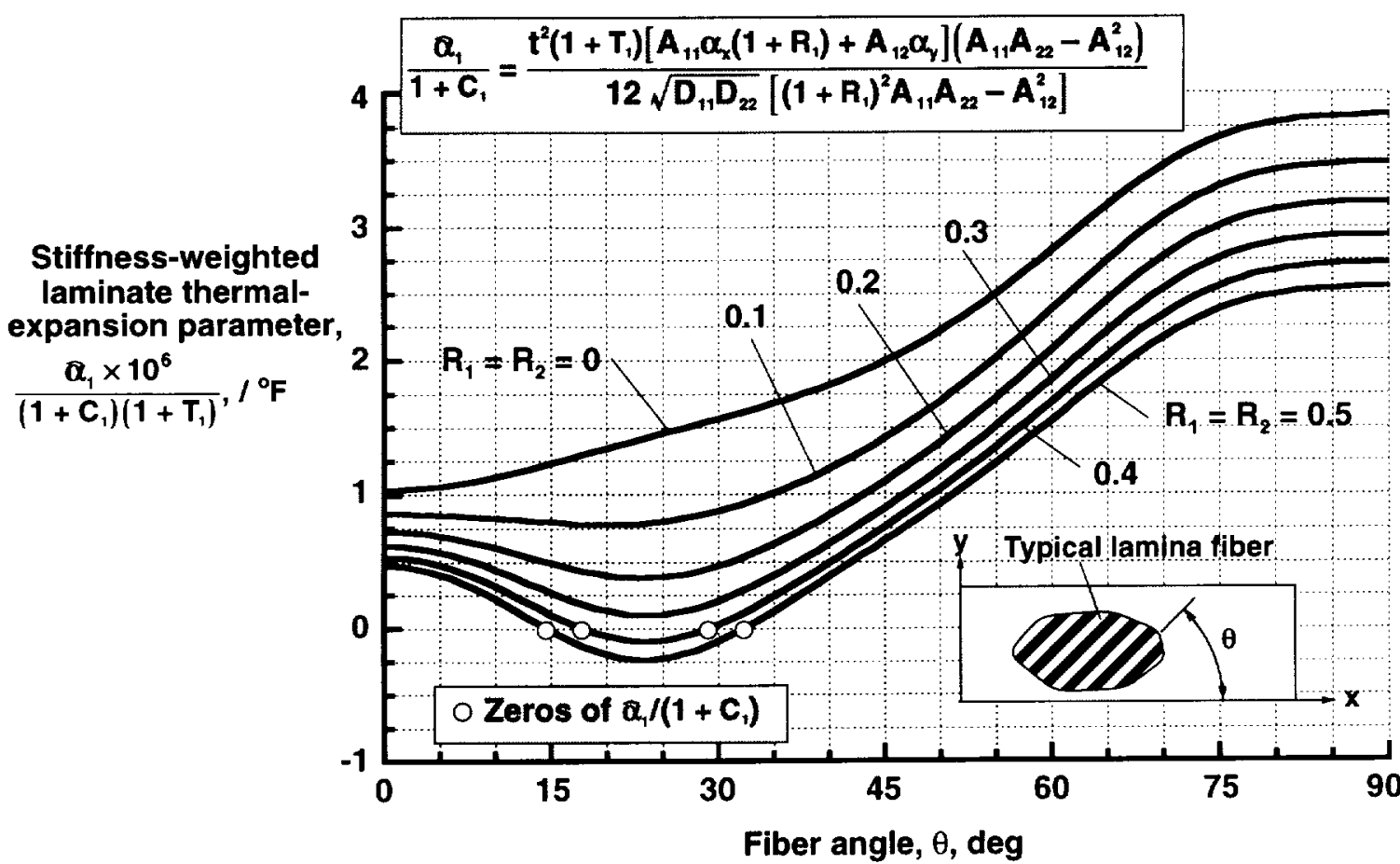

Fig. 10 Stiffness-weighted laminate thermal-expansion parameter $\alpha_{1} /\left[\left(1+C_{1}\right)\left(1+T_{1}\right)\right]$ for $\left[( \pm \theta)_{m} l_{s}\right.$ balanced, angle-ply laminates made of IM7/5260 material and elastically restrained from thermal expansion or contraction by edge plates $\left(R_{2}=R_{1}, T_{1}=T_{2}, m=1,2, \ldots\right)$.

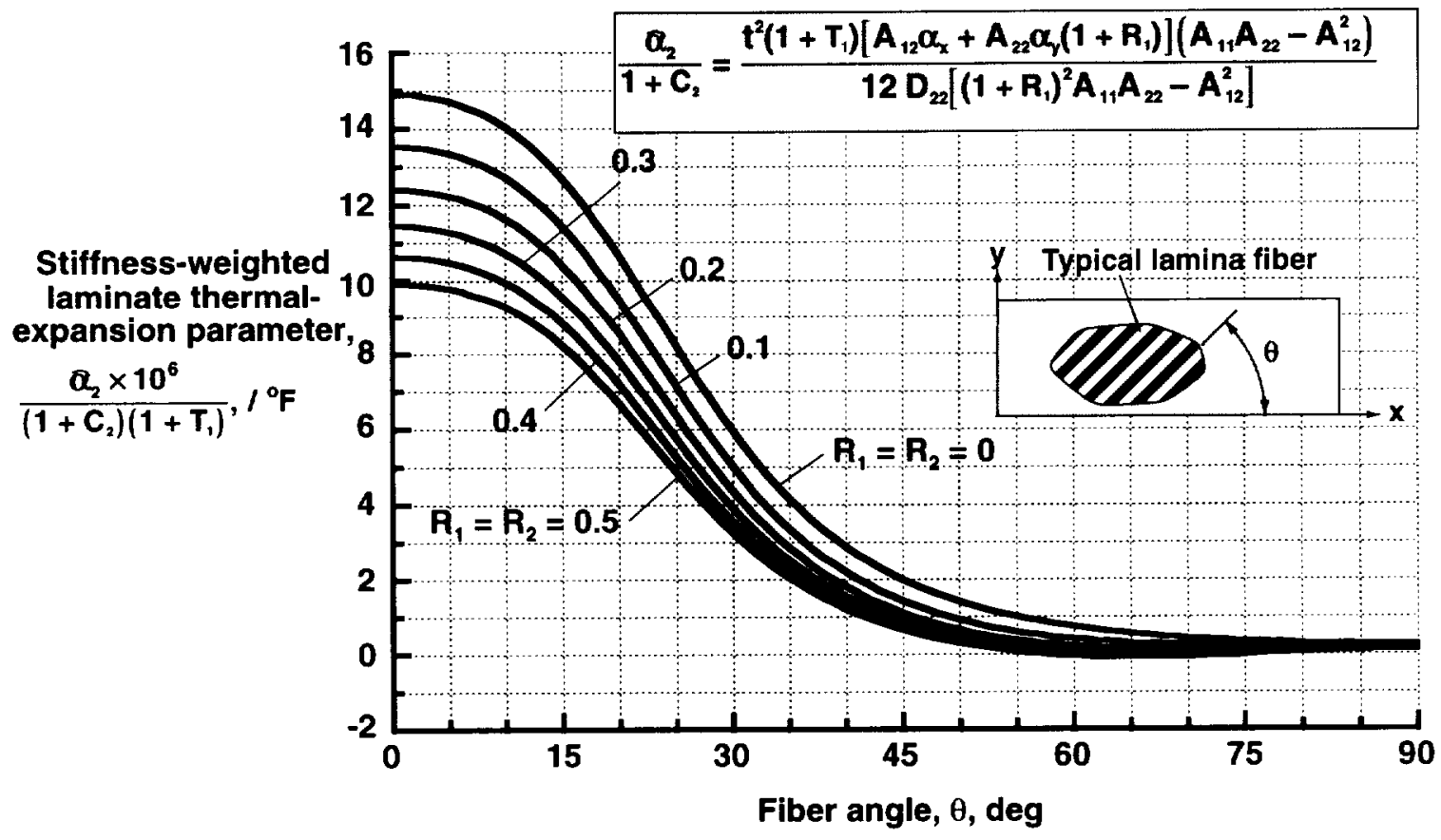

Fig. 11 Stiffness-weighted laminate thermal-expansion parameter $Q_{2} /\left[\left(1+C_{2}\right)\left(1+T_{1}\right)\right]$ for $\left[( \pm \theta)_{m}\right]_{s}$ balanced, angle-ply laminates made of $I M 7 / 5260$ material and elastically restrained from thermal expansion or contraction by edge plates $\left(R_{2}=R_{1}, T_{1}=T_{2}, m=1,2, \ldots\right)$. 


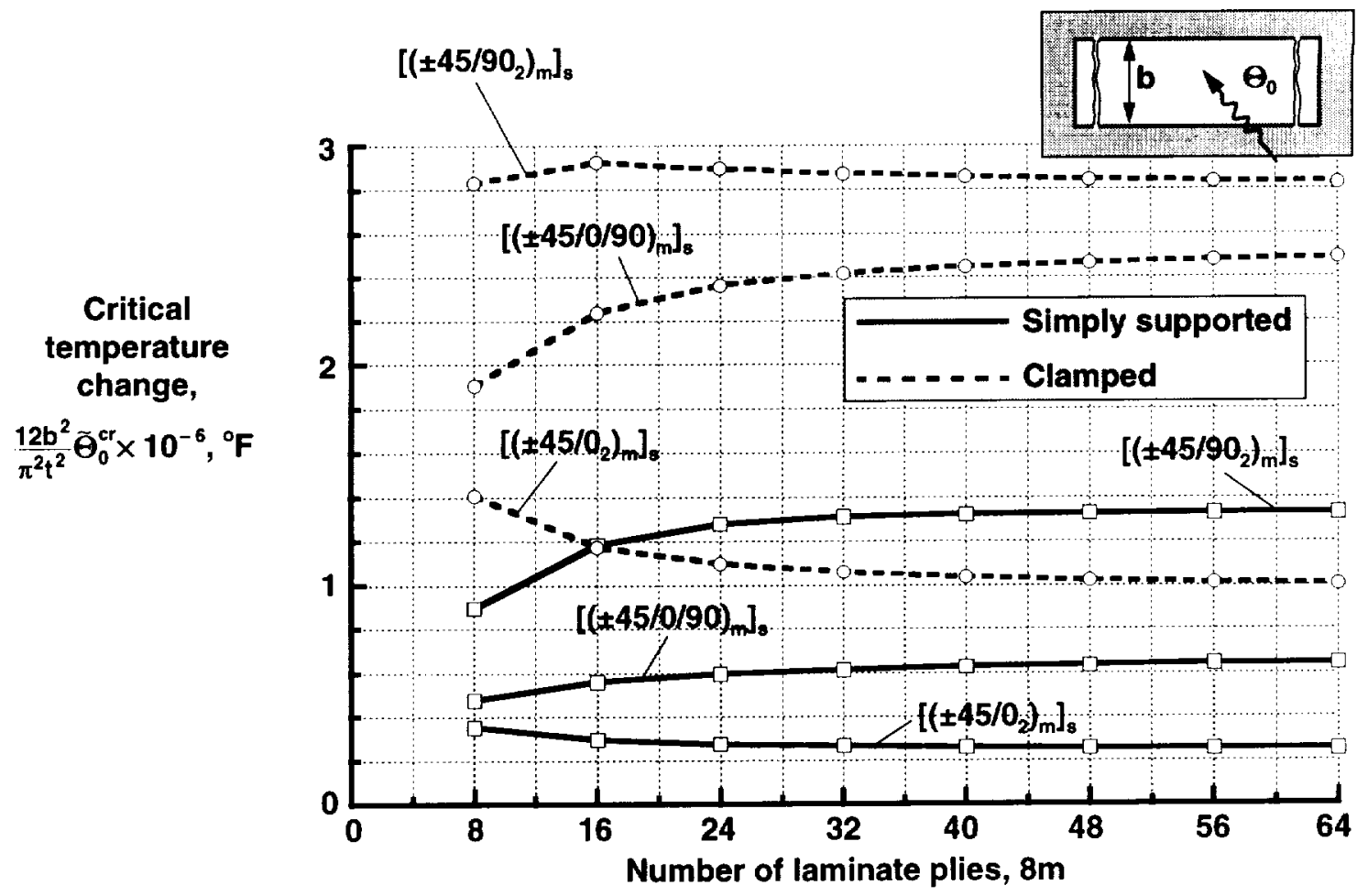

Fig. 12 Critical temperature change for simply supported and clamped laminates made of IM7/5260 material, fully restrained against thermal expansion, and subjected to uniform heating.

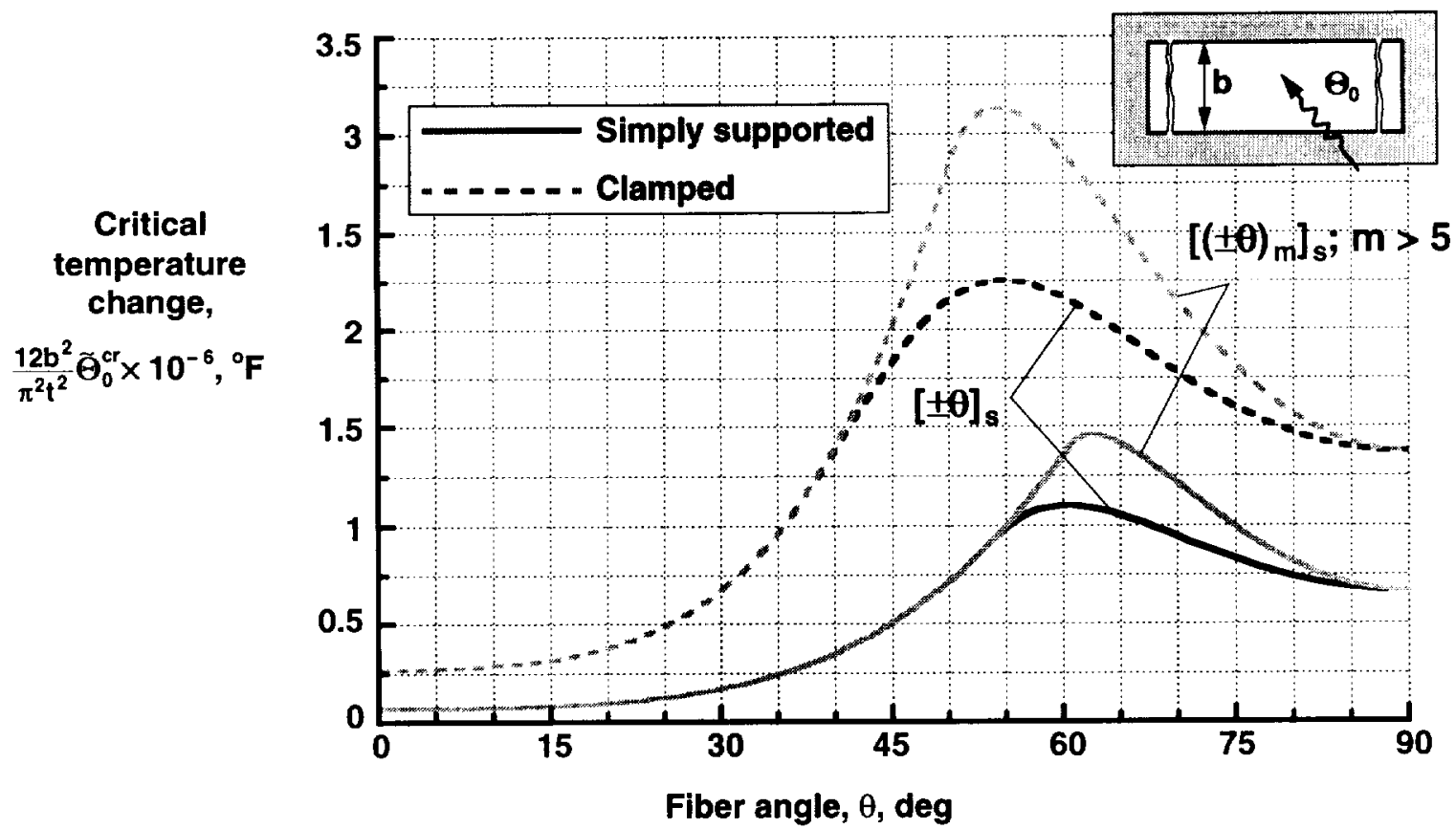

Fig. 13 Critical temperature change for simply supported and clamped angle-ply laminates made of IM7/5260 material, fully restrained against thermal expansion, and subjected to uniform heating $(m=1,2, \ldots)$. 


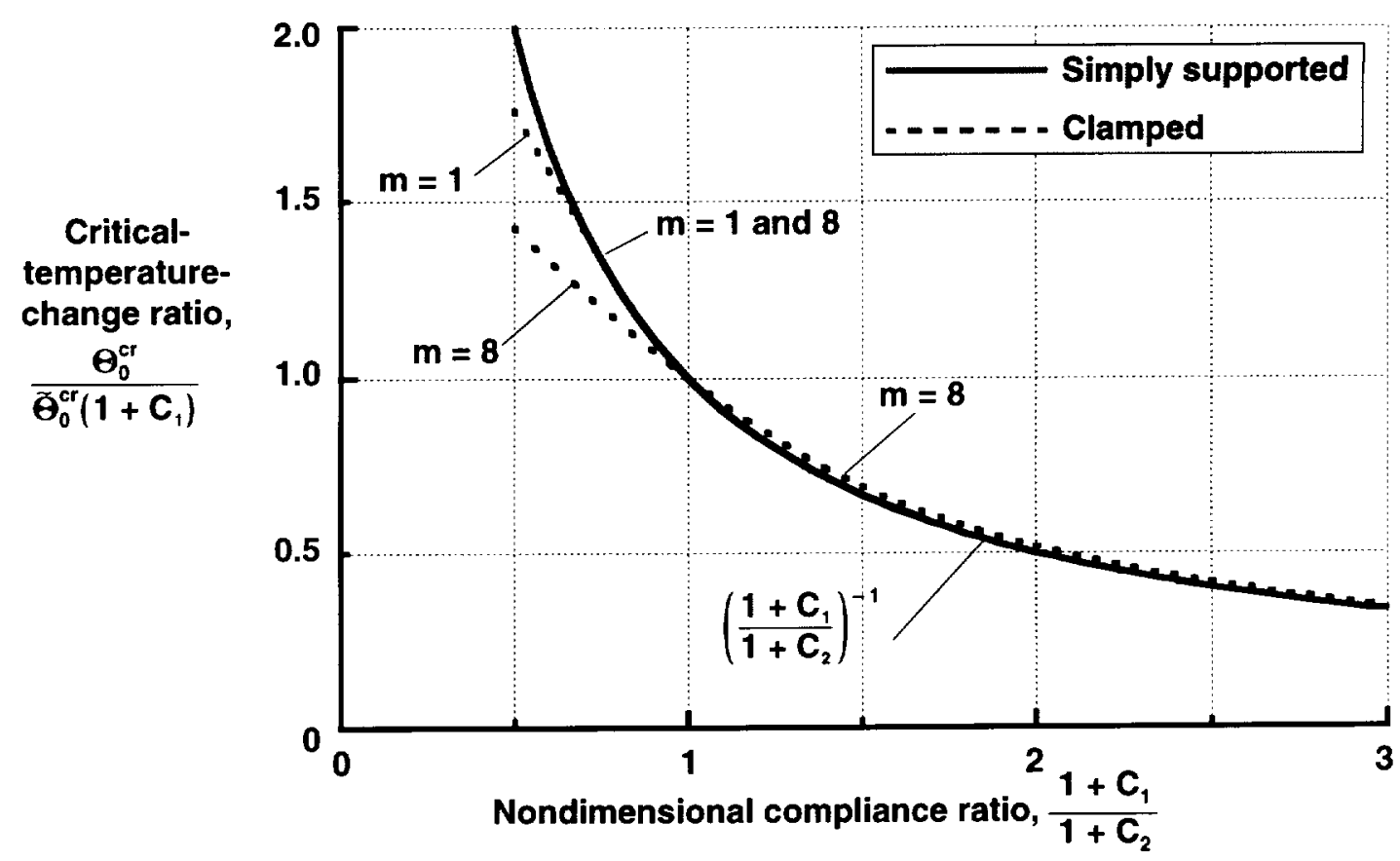

Fig. 14 Critical-temperature-change ratio as a function of the nondimensional compliance ratio $\left(1+C_{1}\right) /\left(1+C_{2}\right)$ for $\left[( \pm 45 / 0 / 90)_{m}\right]_{s}$ laminates made of IM7/5260 material and elastically restrained from thermal expansion or contraction by edge plates.

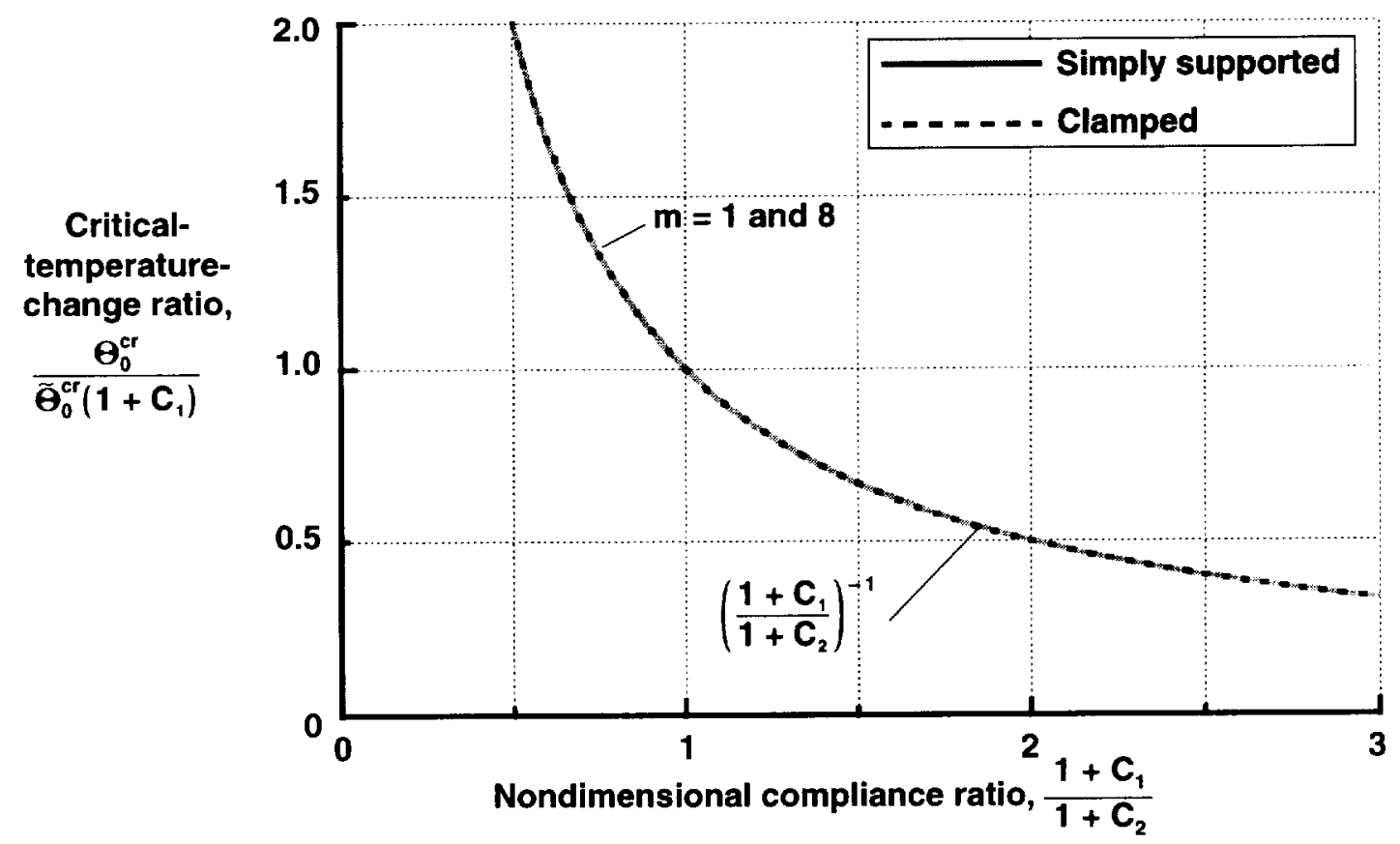

Fig. 15 Critical-temperature-change ratio as a function of the nondimensional compliance ratio $\left(1+C_{1}\right) /\left(1+C_{2}\right)$ for $\left[\left( \pm 45 / 0_{2}\right)_{\mathrm{m}}\right]_{\mathrm{s}}$ laminates made of $\mathrm{IM} 7 / 5260$ material and elastically restrained from thermal expansion or contraction by edge plates. 


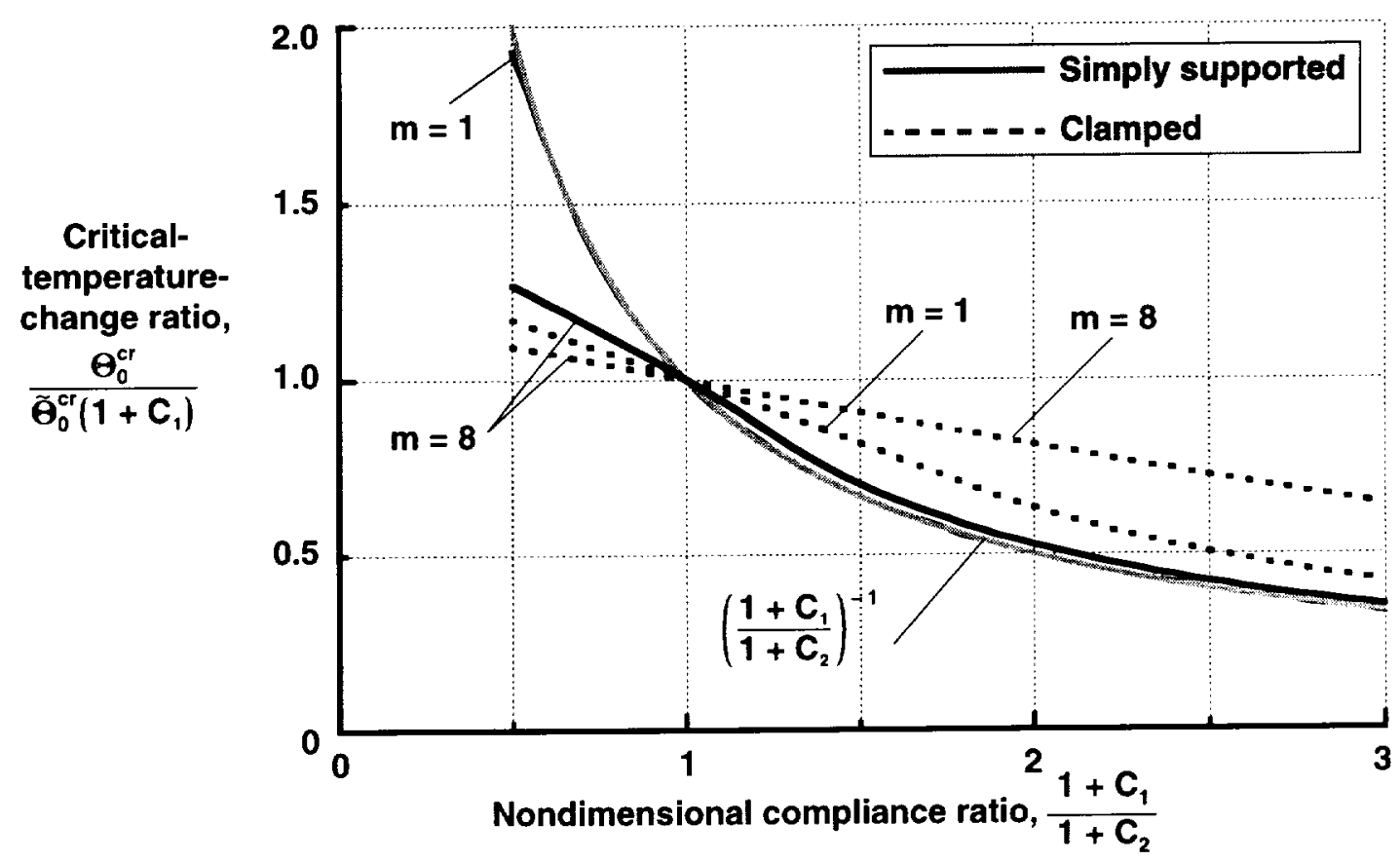

Fig. 16 Critical-temperature-change ratio as a function of the nondimensional compliance ratio $\left(1+C_{1}\right) /\left(1+C_{2}\right)$ for $\left[\left( \pm 45 / 90_{2}\right)_{m}\right]_{s}$ laminates made of IM7/5260 material and elastically restrained from thermal expansion or contraction by edge plates.

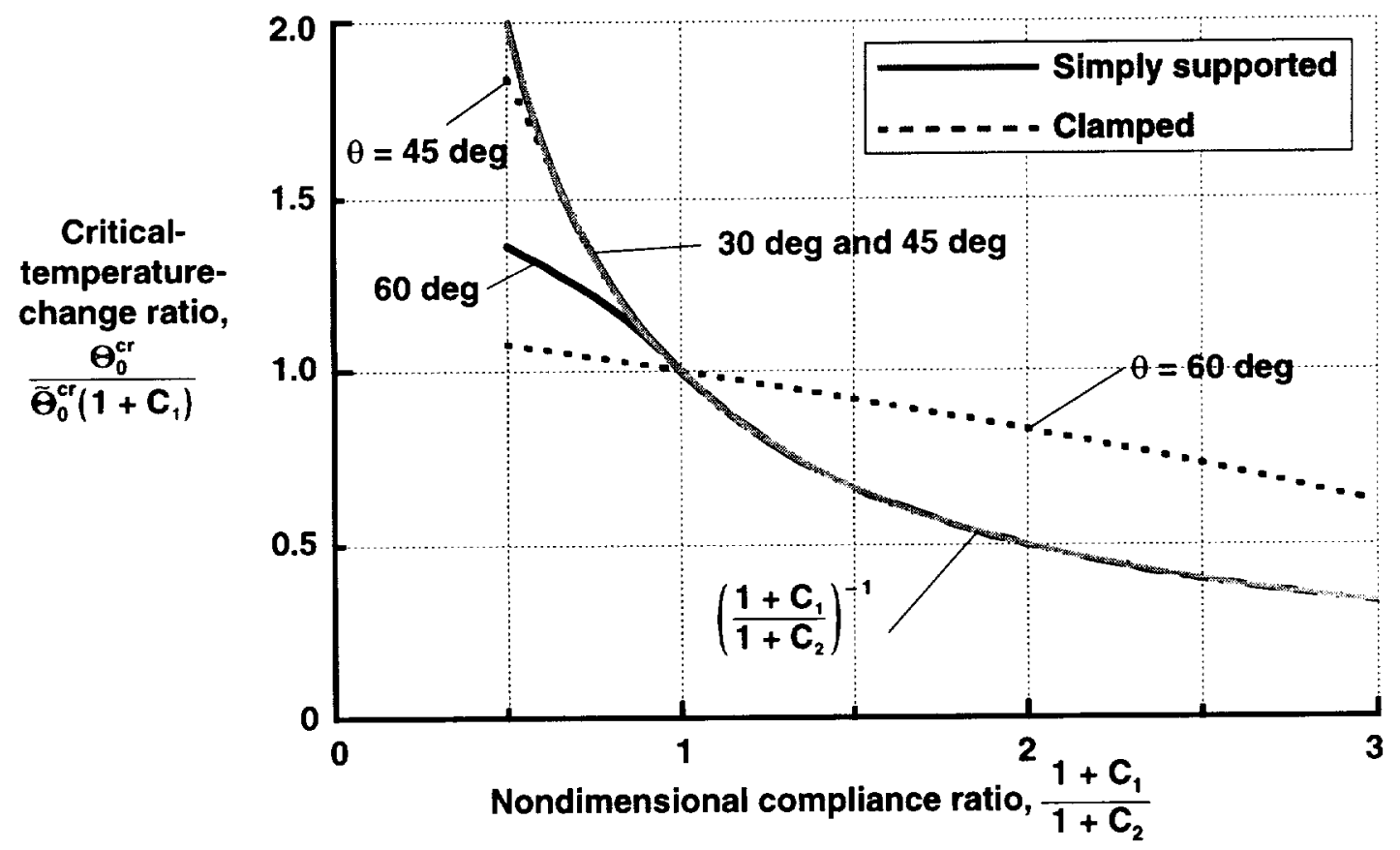

Fig. 17 Critical-temperature-change ratio as a function of the nondimensional compliance ratio $\left(1+C_{1}\right) /\left(1+C_{2}\right)$ for $\left[( \pm \theta)_{m}\right]_{s}$ laminates $(m>5)$ made of IM7/5260 material and elastically restrained from thermal expansion or contraction by edge plates. 


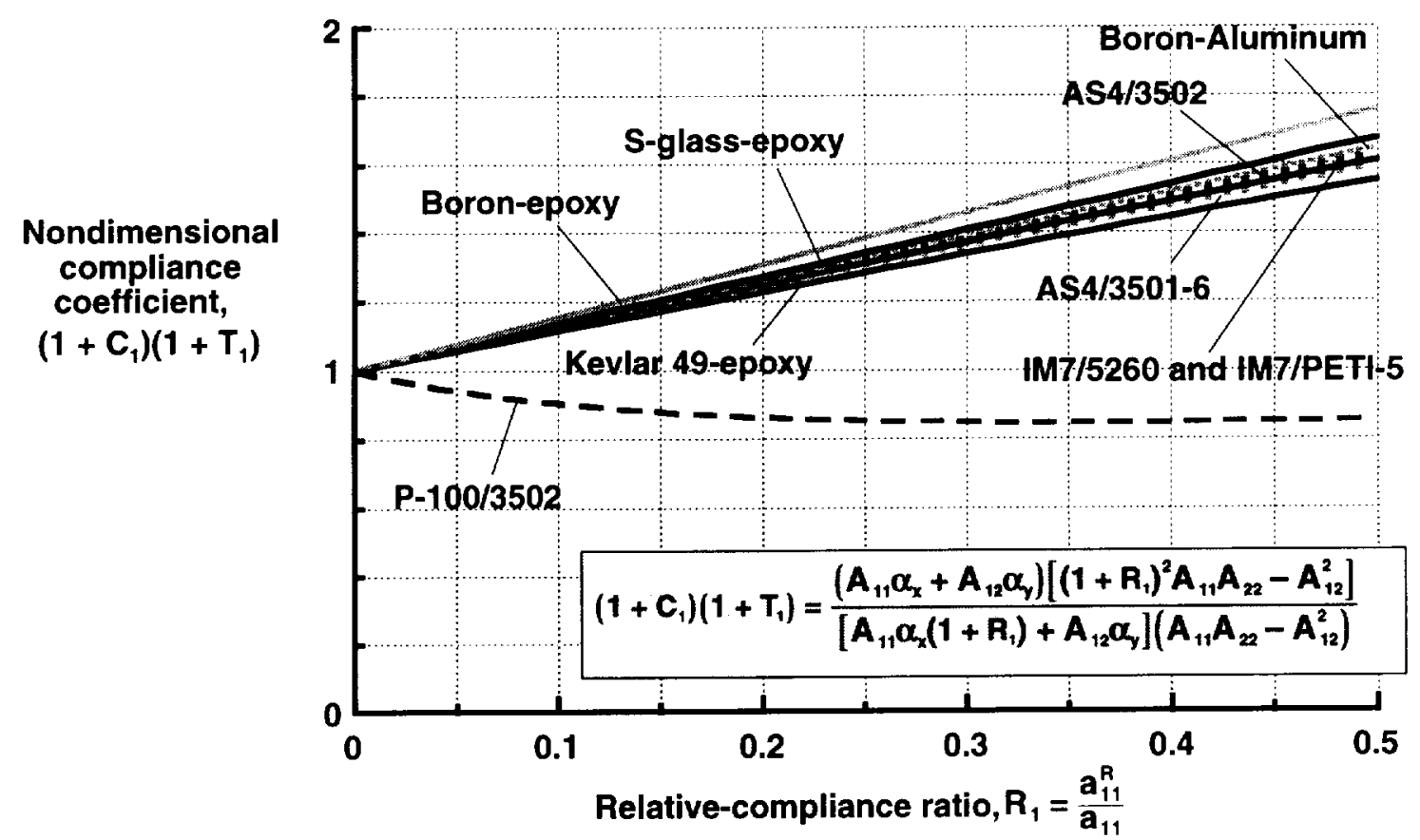

Fig. 18 Effects of lamina material properties on nondimensional compliance coefficient $\left(1+C_{1}\right)\left(1+T_{1}\right)$ for $\left[\left( \pm 45 / 90,{ }_{2}\right]\right.$ laminates elastically restrained from thermal expansion or contraction by edge plates $\left(R_{2}=R_{1}, T_{1}=T_{2}\right.$, and $\left.m=1,2, \ldots\right)$.

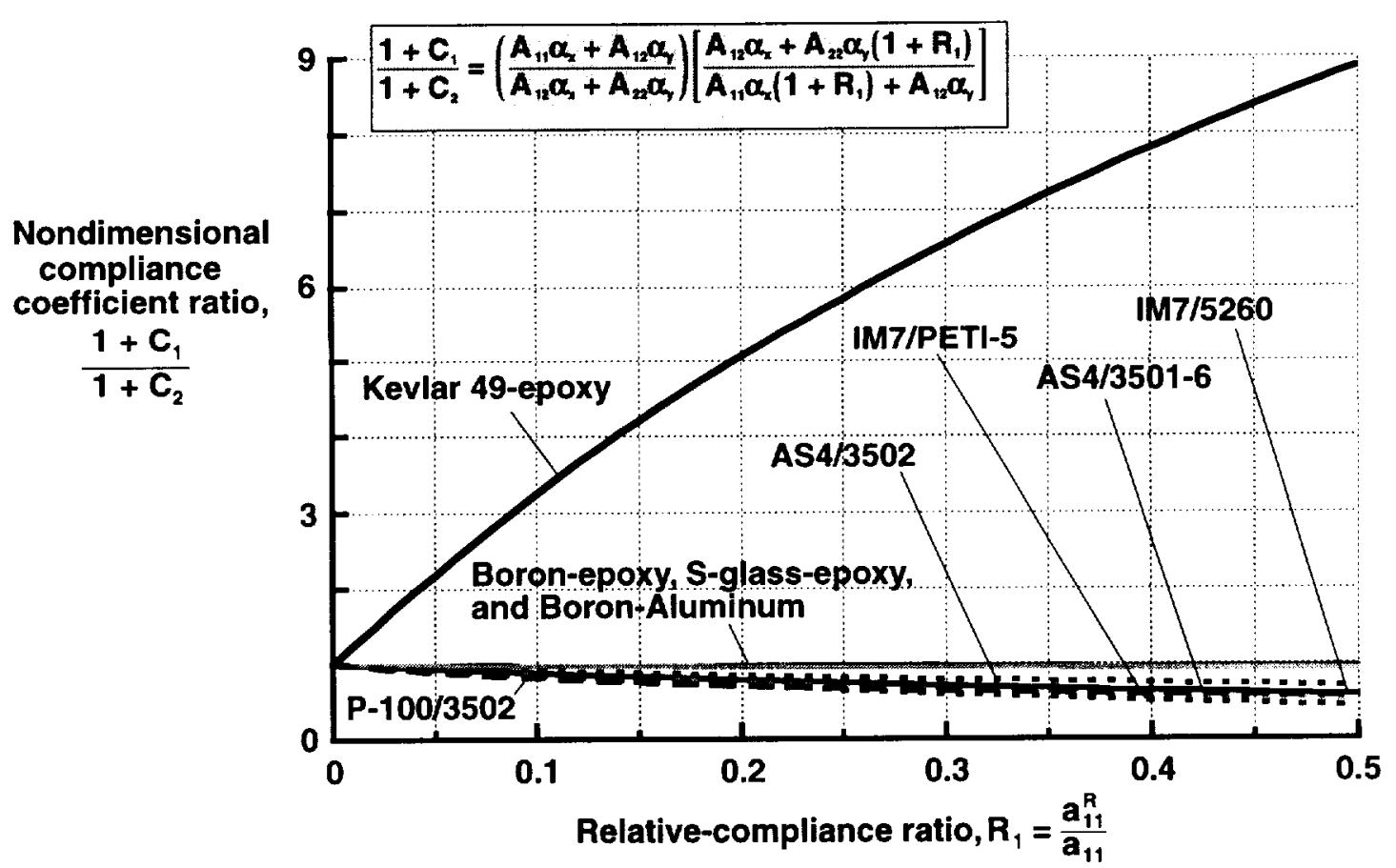

Fig. 19a Effects of lamina material properties on nondimensional compliance coefficient ratio $\left(1+C_{1}\right) /\left(1+C_{2}\right)$ for $\left[( \pm 45 / 90,)_{m}\right]_{\mathrm{s}}$ laminates elastically restrained from thermal expansion or contraction by edge plates $\left(R_{2}=R_{1}, T_{1}=T_{2}\right.$, and $\left.m=1,2, \ldots\right)$. 


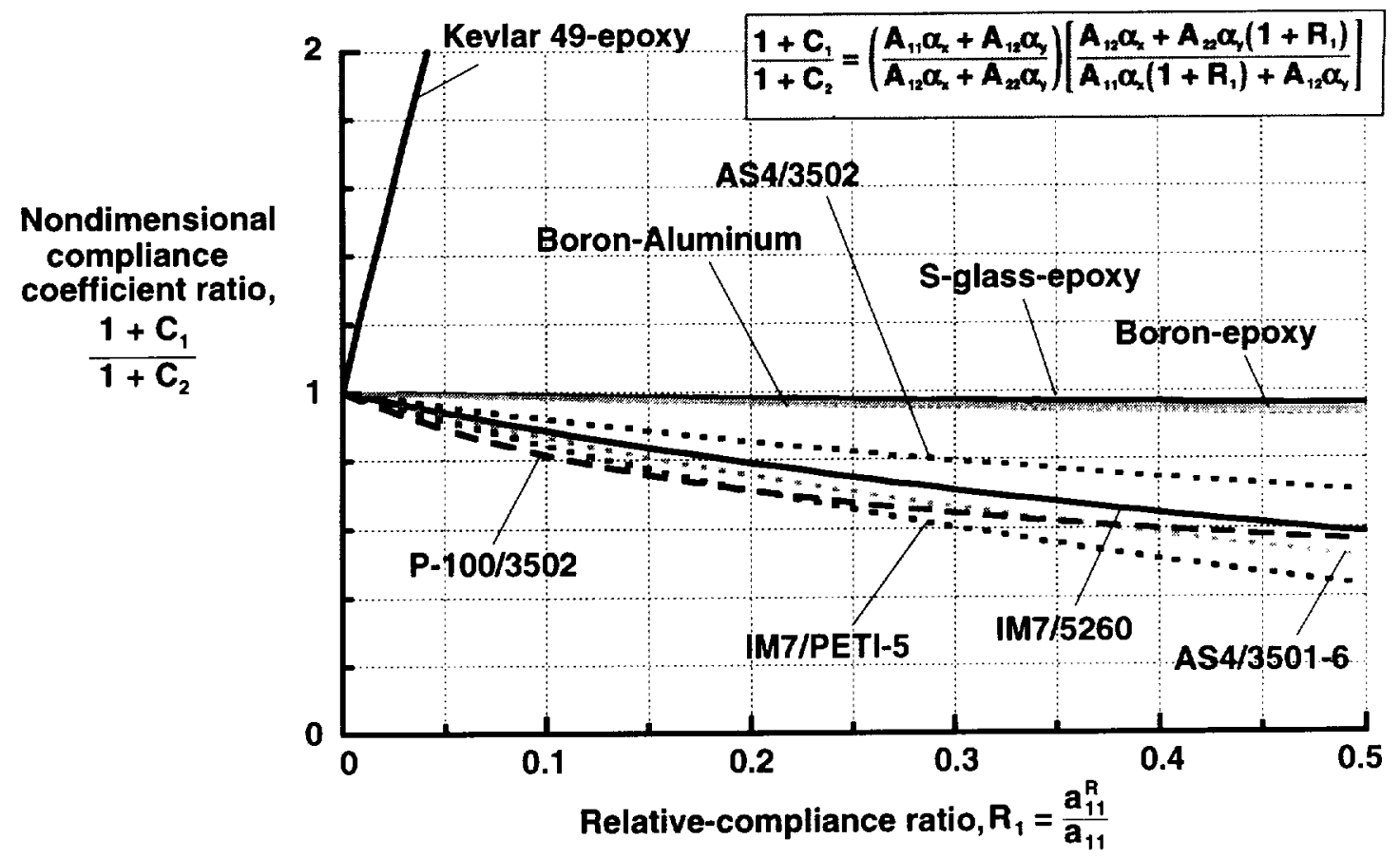

Fig. 19b Effects of lamina material properties on nondimensional compliance coefficient ratio $\left(1+C_{1}\right) /\left(1+C_{2}\right)$ for $[( \pm 45 / 90)]$ laminates elastically restrained from thermal expansion or contraction by edge plates $\left(R_{2}=R_{1}, T_{1}=T_{2}\right.$, and $\left.m=1,2, \ldots\right)$.

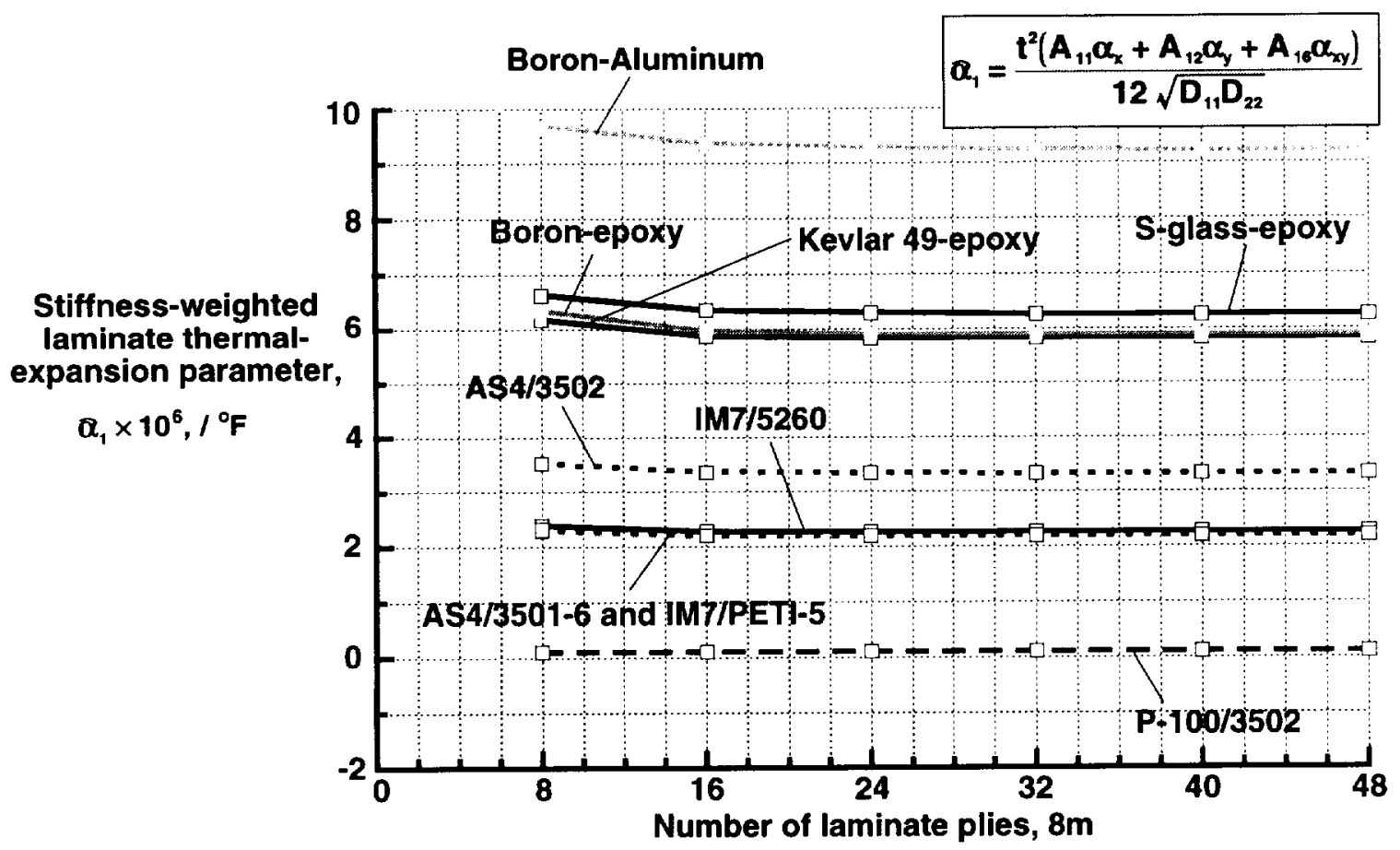

Fig. 20 Effects of lamina material properties on stiffness-weighted laminate thermal-expansion parameter $\boldsymbol{\alpha}_{1}$ for $\left[( \pm 45 / 90)_{2}\right]_{\mathrm{s}}$ laminates. 


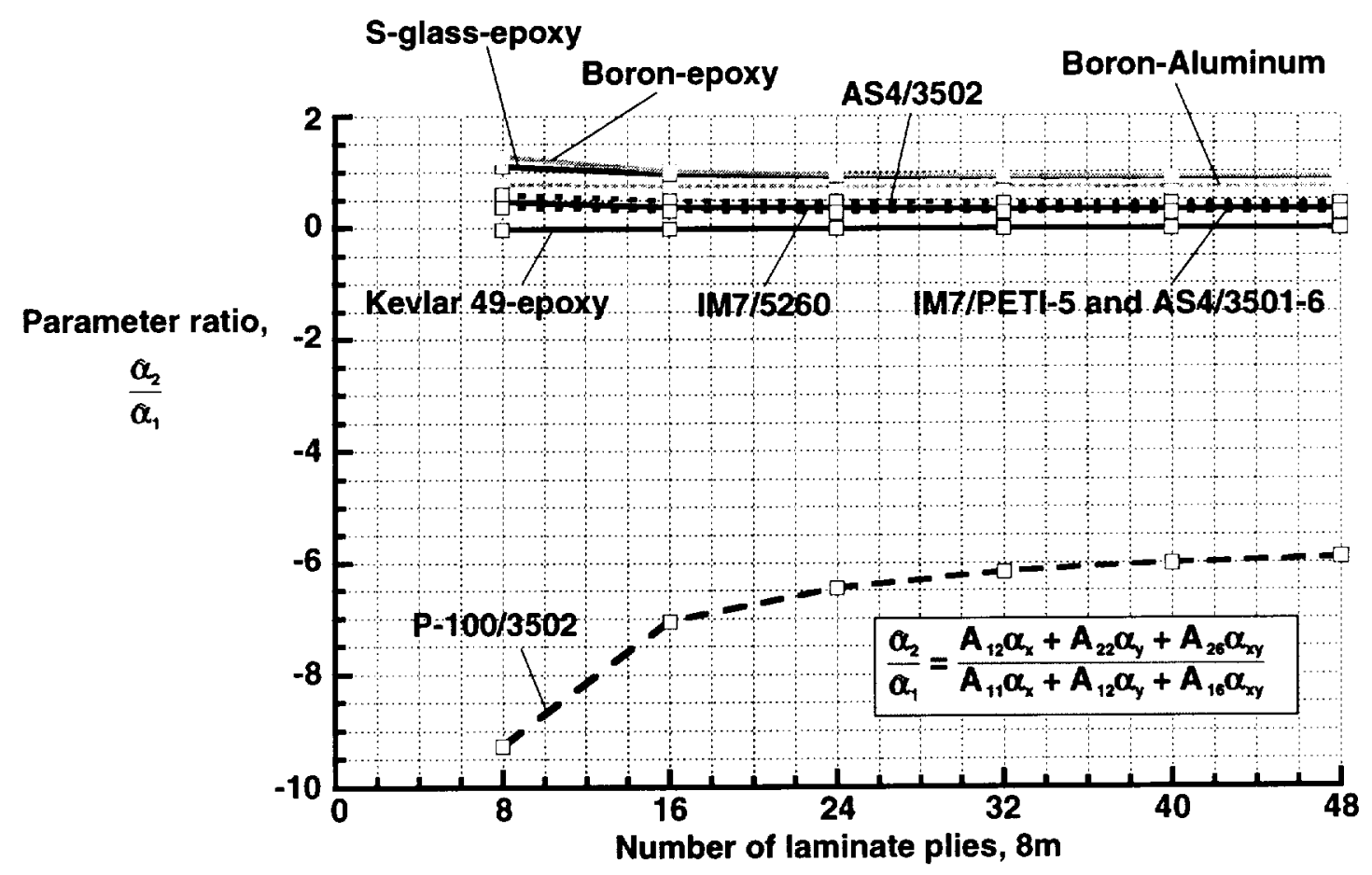

Fig. 21a Effects of lamina material properties on parameter ratio $\alpha_{2} / \alpha_{1}$ for $\left[\left( \pm 45 / 90_{2}\right)_{m}\right]_{s}$ laminates.

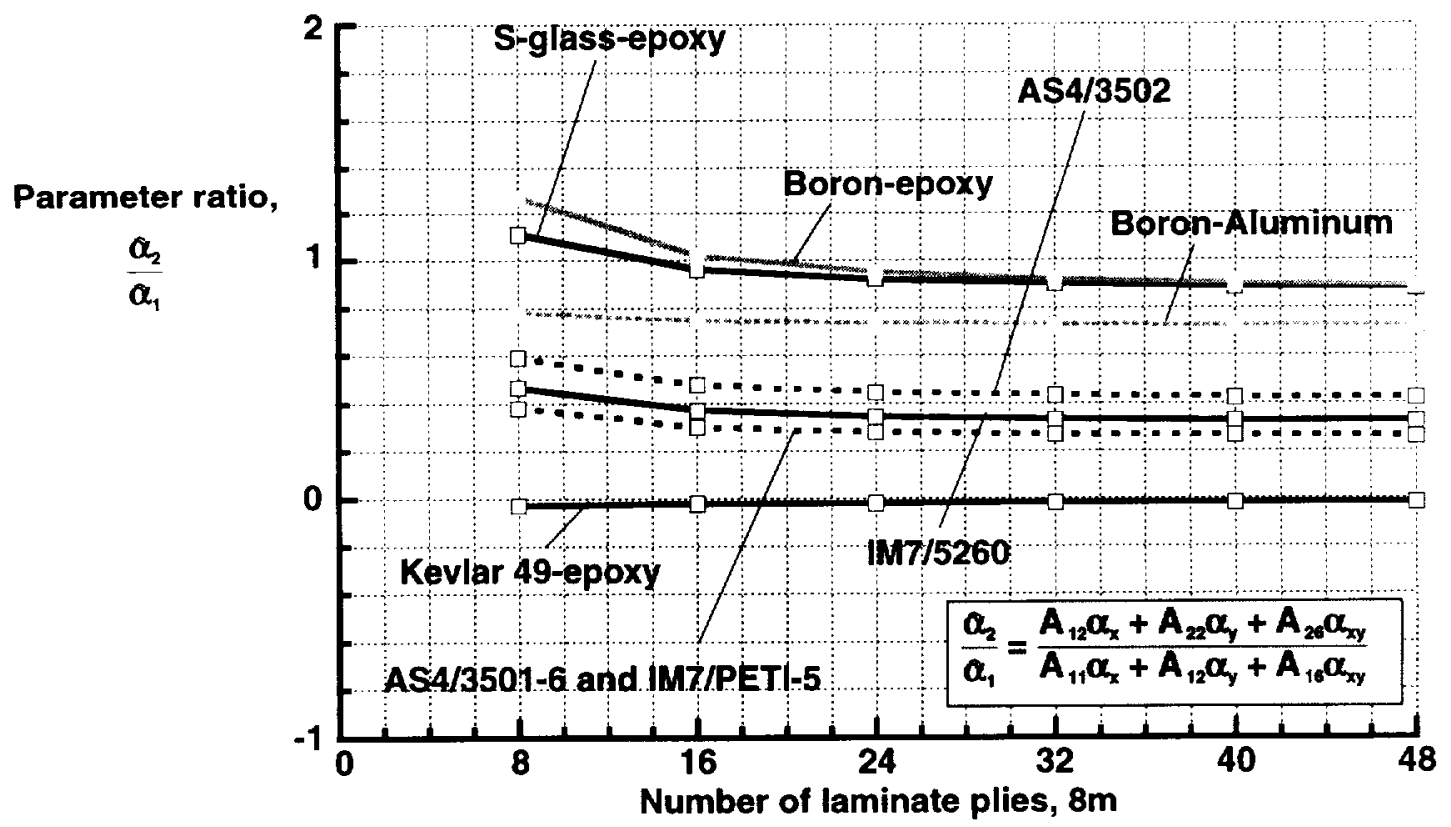

Fig. 21b Effects of lamina material properties on parameter ratio $\alpha_{2} / \alpha_{1}$ for $\left[\left( \pm 45 / 90_{2}\right)_{\mathrm{m}}\right]_{\mathrm{s}}$ laminates. 


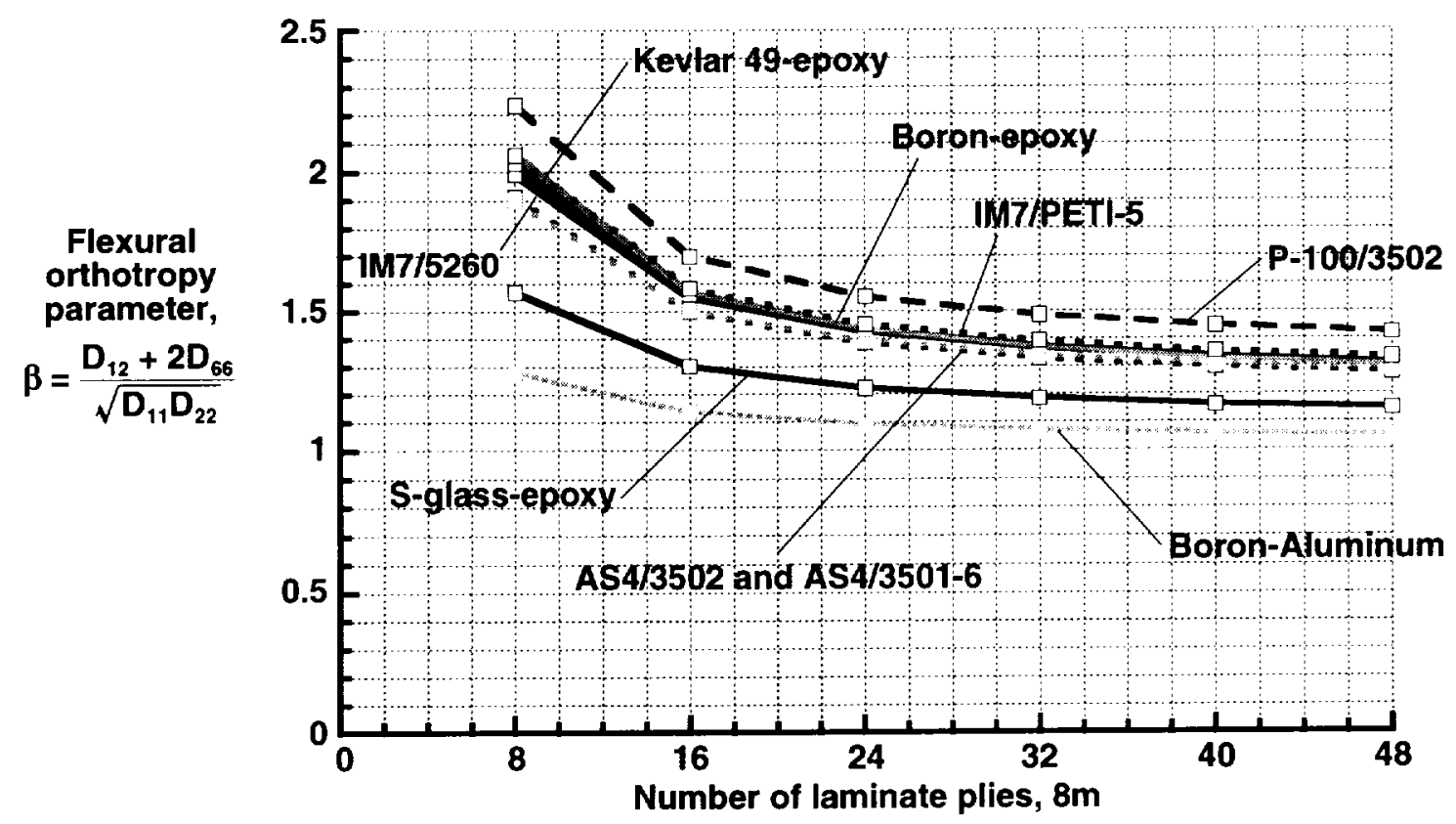

Fig. 22 Effects of lamina material properties on nondimensional flexural orthotropy parameter $\beta$ for $\left[( \pm 45 / 90,)_{\mathrm{m}}\right]_{\mathrm{s}}$ and $\left[\left( \pm 45 / 0_{2}\right)_{m}\right]_{s}$ laminates.

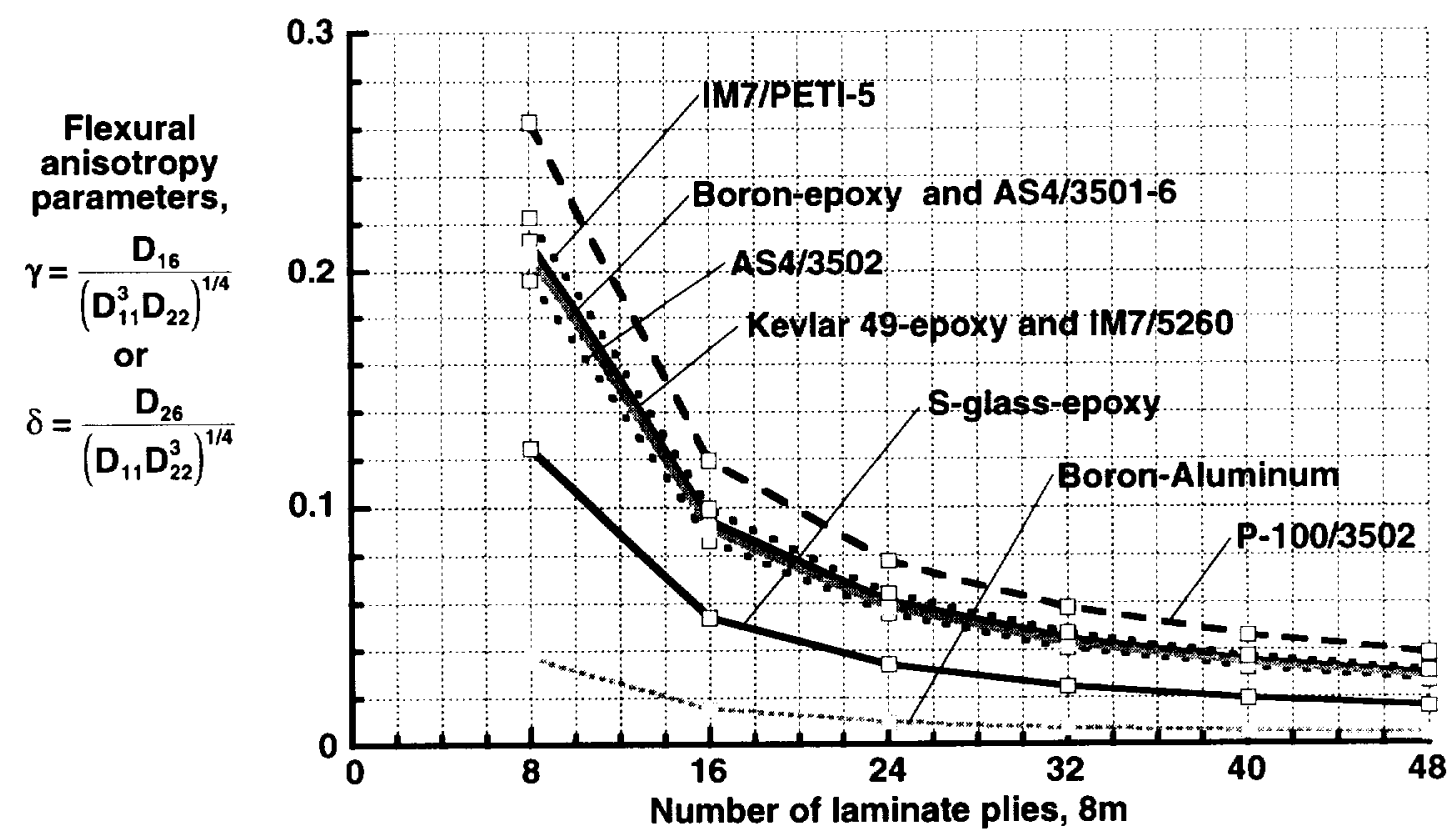

Fig. 23 Effects of lamina material properties on nondimensional flexural anisotropy parameter $\gamma$ for $\left[\left( \pm 45 / 90_{2}\right)_{\mathrm{m}}\right]_{\mathrm{s}}$ laminates and $\delta$ for $\left[\left( \pm 45 / 0_{2}\right)_{\mathrm{m}}\right]_{\mathrm{s}}$ laminates. 


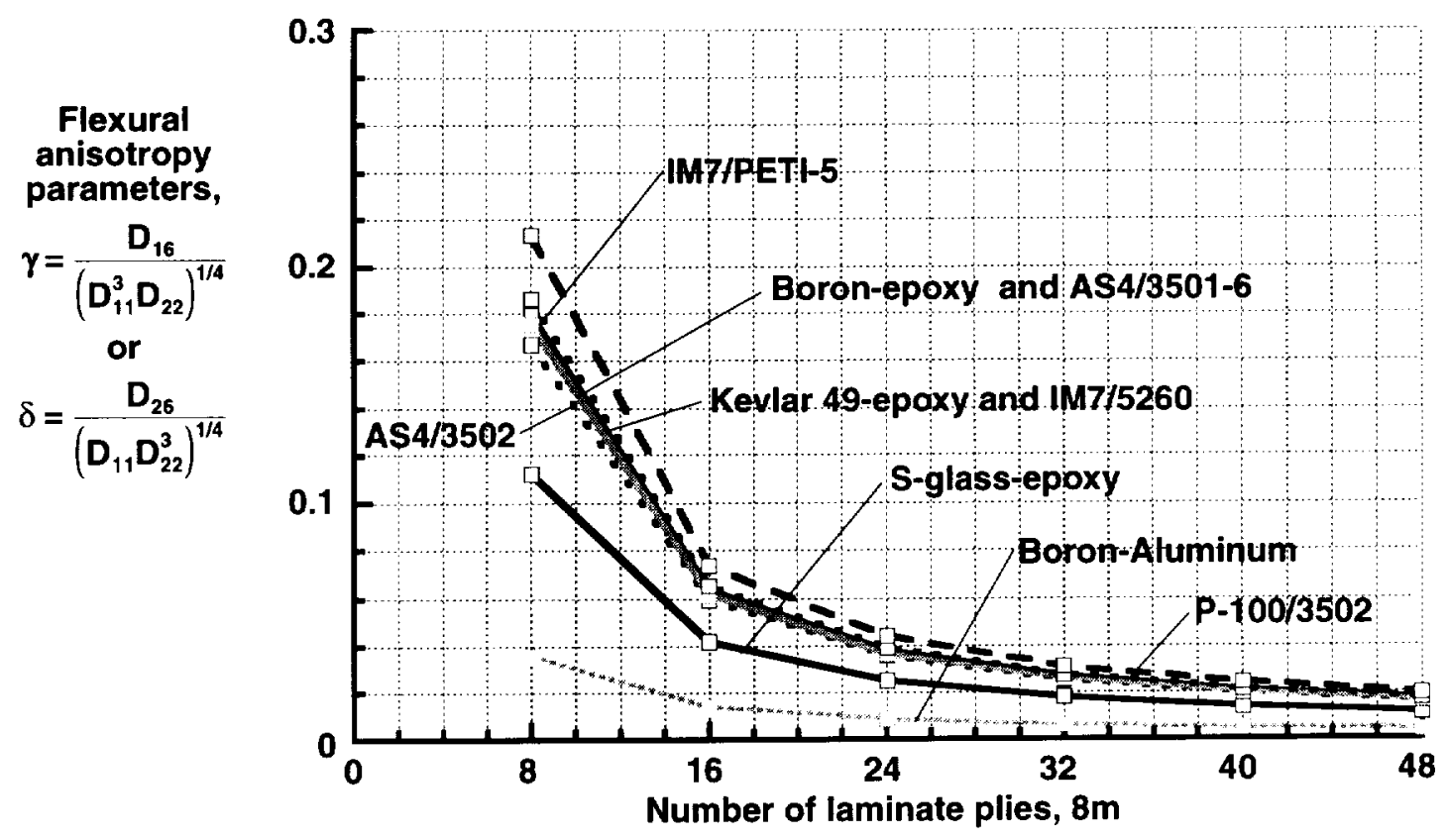

Fig. 24 Effects of lamina material properties on nondimensional flexural anisotropy parameter $\delta$ for $\left[\left( \pm 45 / 90_{2}\right)_{\mathrm{m}}\right]_{\mathrm{s}}$ laminates and $\gamma$ for $\left[\left( \pm 45 / 0_{2}\right)_{m}\right]_{s}$ laminates.

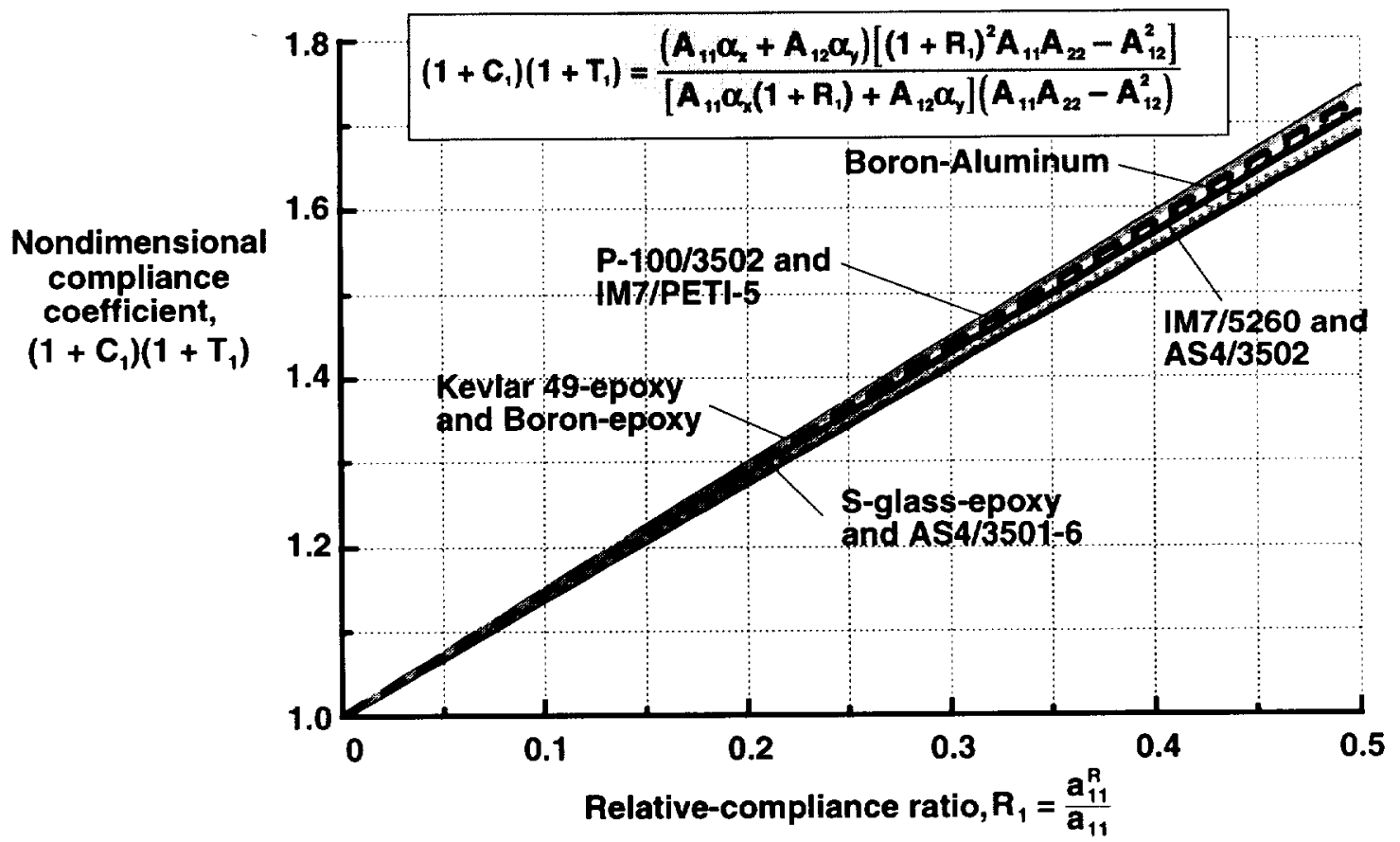

Fig. 25 Effects of lamina material properties on nondimensional compliance coefficient $\left(1+C_{1}\right)\left(1+T_{1}\right)$ for $\left[( \pm 45 / 0 / 90)_{m}\right]_{s}$ laminates elastically restrained from thermal expansion or contraction by edge plates $\left(\mathbf{R}_{2}=\mathbf{R}_{1}, \mathbf{T}_{1}=\mathbf{T}_{2}\right.$, and $\left.\mathrm{m}=1,2, \ldots\right)$. 


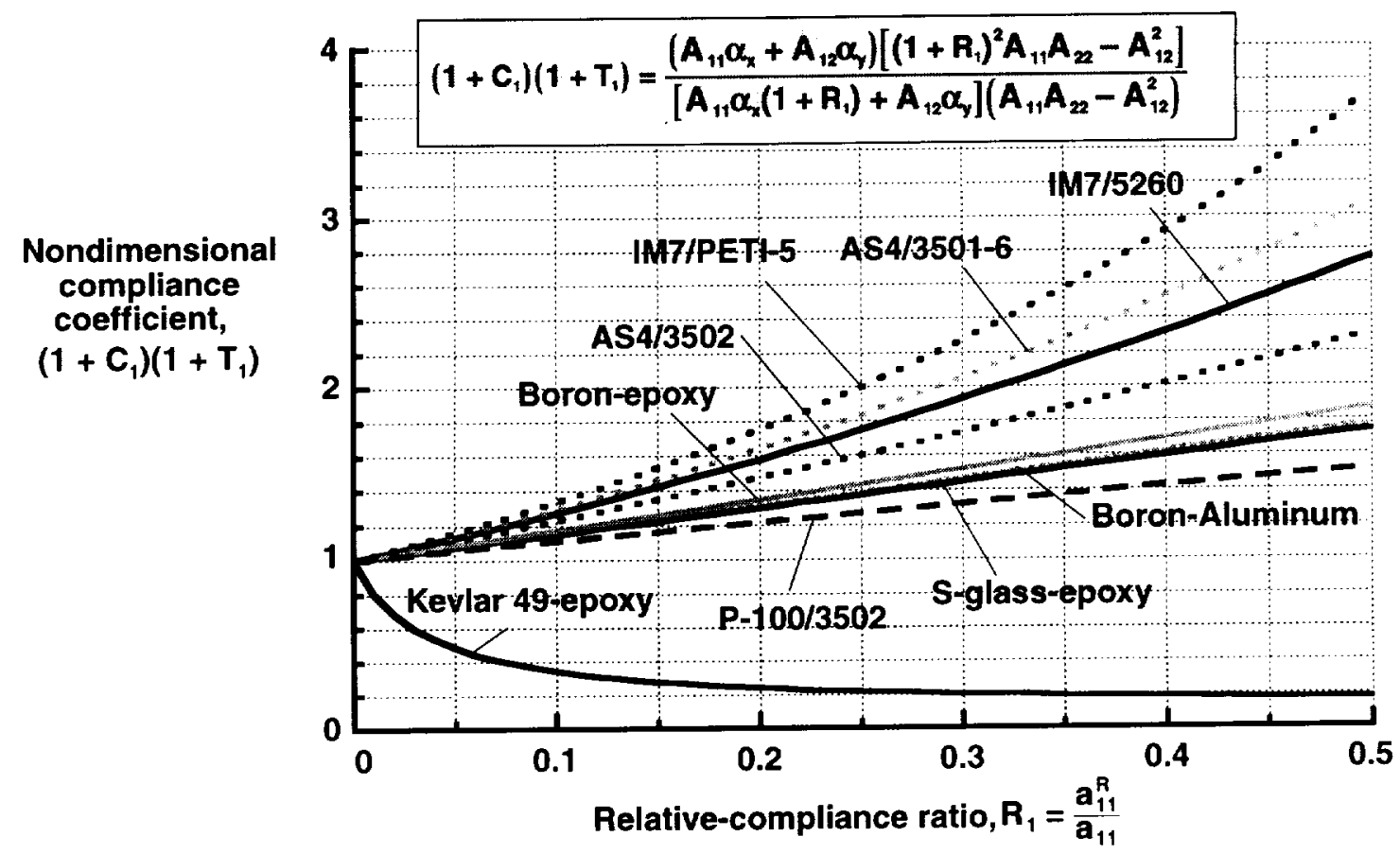

Fig. 26 Effects of lamina material properties on nondimensional compliance coefficient $\left(1+C_{1}\right)\left(1+T_{1}\right)$ for $[( \pm 45 / 0)]$ laminates elastically restrained from thermal expansion or contraction by edge plates $\left(R_{2}=R_{1}, T_{1}=T_{2}\right.$, and $\left.m=1,2, \ldots\right)$.

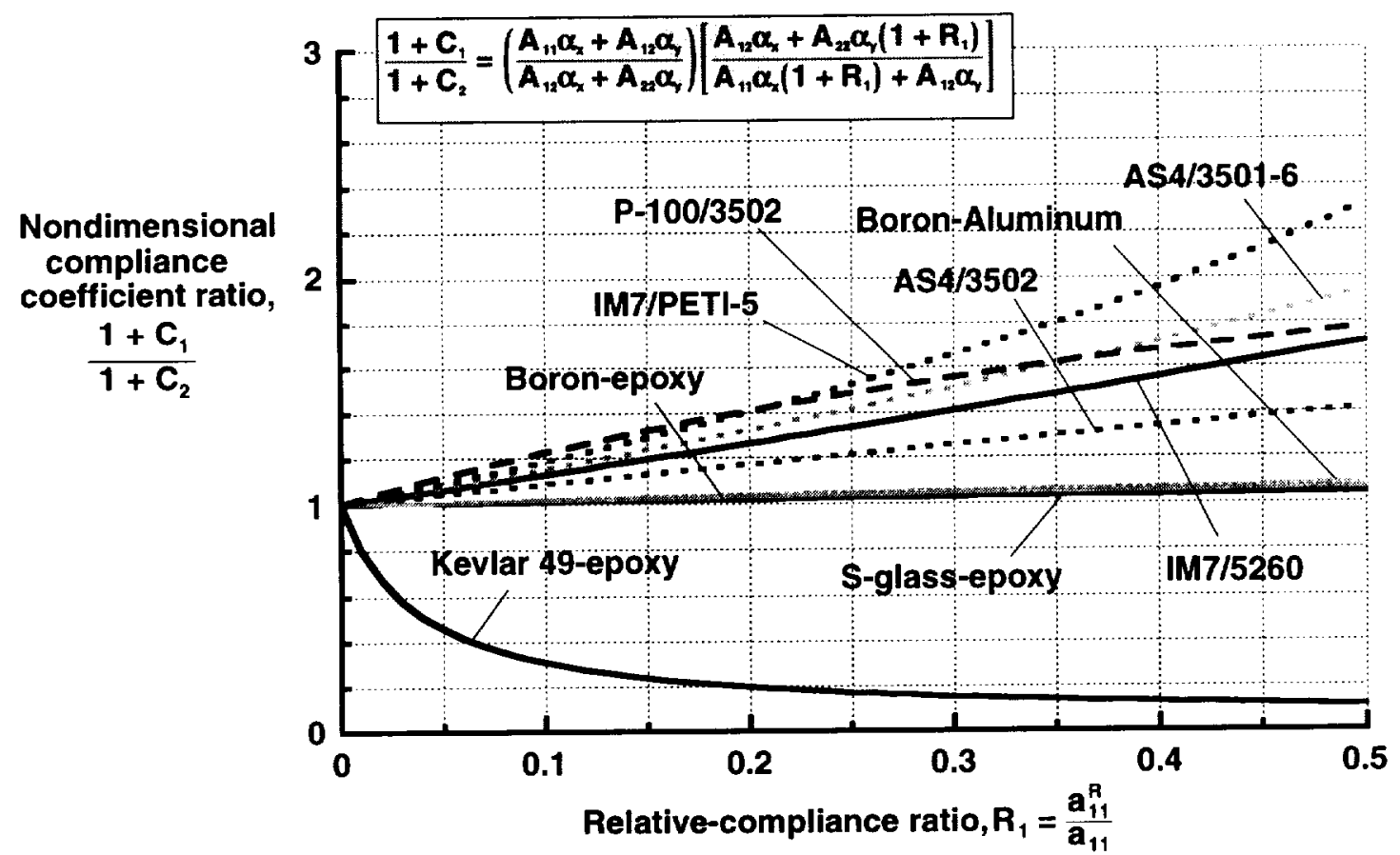

Fig. 27 Effects of lamina material properties on nondimensional compliance coefficient ratio $\left(1+C_{1}\right) /\left(1+C_{2}\right)$ for $\left[\left( \pm 45 / 0_{2}\right)_{\mathrm{m}}\right]_{\mathrm{s}}$ laminates elastically restrained from thermal expansion or contraction by edge plates $\left(R_{2}=R_{1}, T_{1}=T_{2}\right.$, and $\left.m=1,2, \ldots\right)$. 


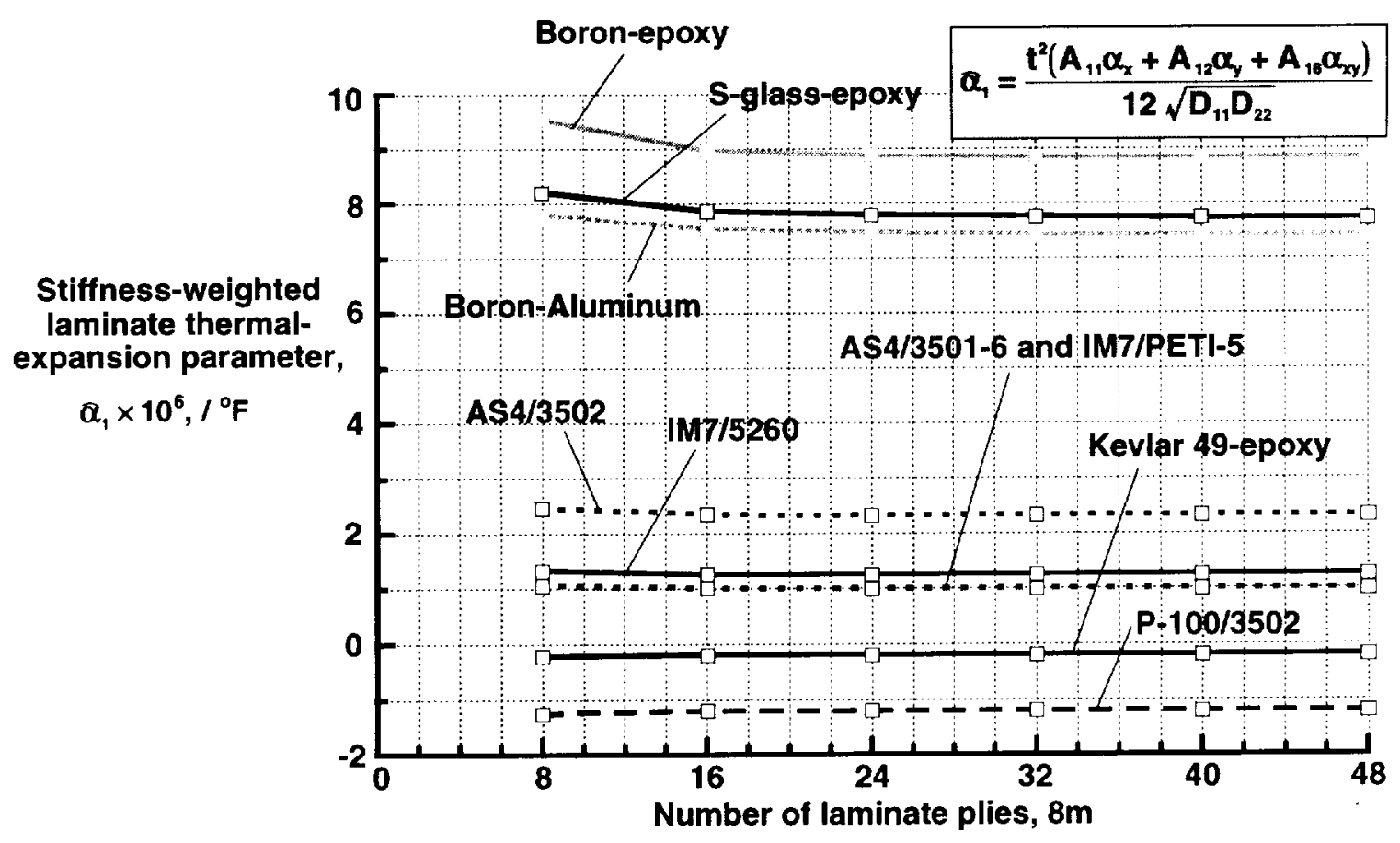

Fig. 28 Effects of lamina material properties on stiffness-weighted laminate thermal-expansion parameter $\hat{\alpha}_{1}$ for $\left[\left( \pm 45 / 0_{2}\right)_{\mathrm{m}}\right]_{\mathrm{s}}$ laminates.

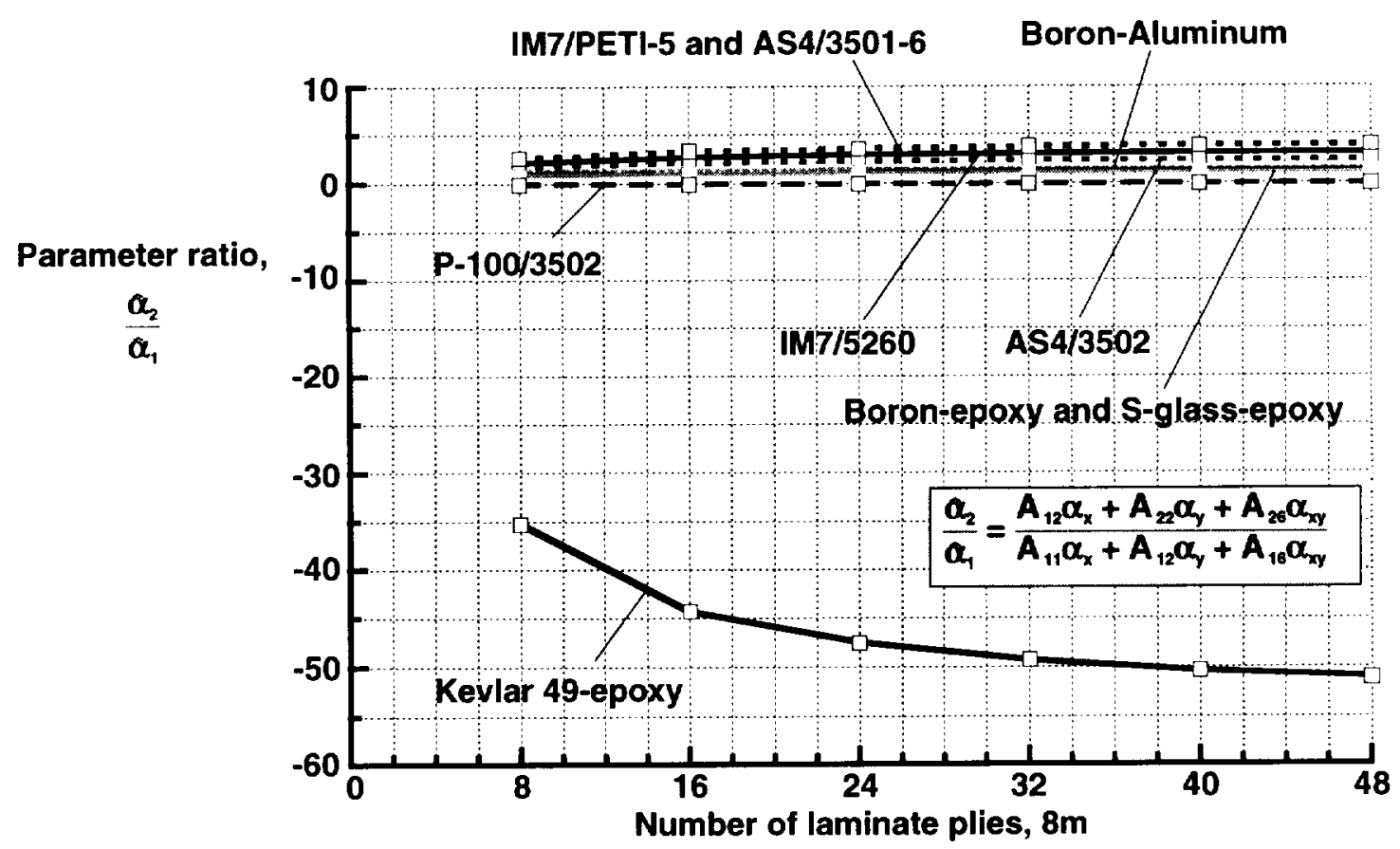

Fig. 29a Effects of lamina material properties on parameter ratio $\alpha_{2} / \alpha_{1}$ for $\left[\left( \pm 45 / 0_{2}\right)_{m}\right]_{s}$ laminates. 


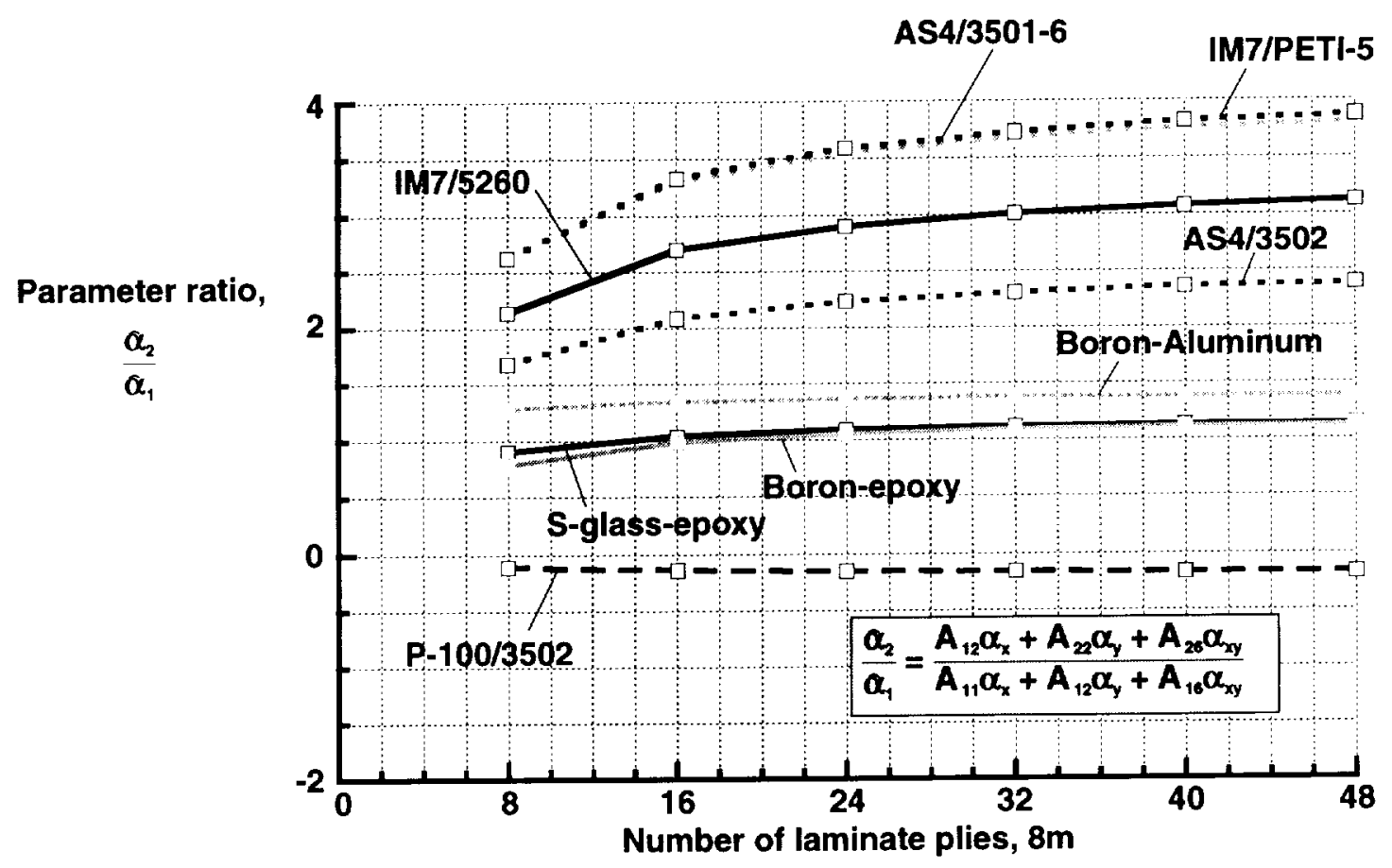

Fig. $29 \mathrm{~b}$ Effects of lamina material properties on parameter ratio $\alpha_{2} / \alpha_{1}$ for $\left[\left( \pm 45 / 0_{2}\right)_{\mathrm{m}}\right]_{\mathrm{s}}$ laminates.

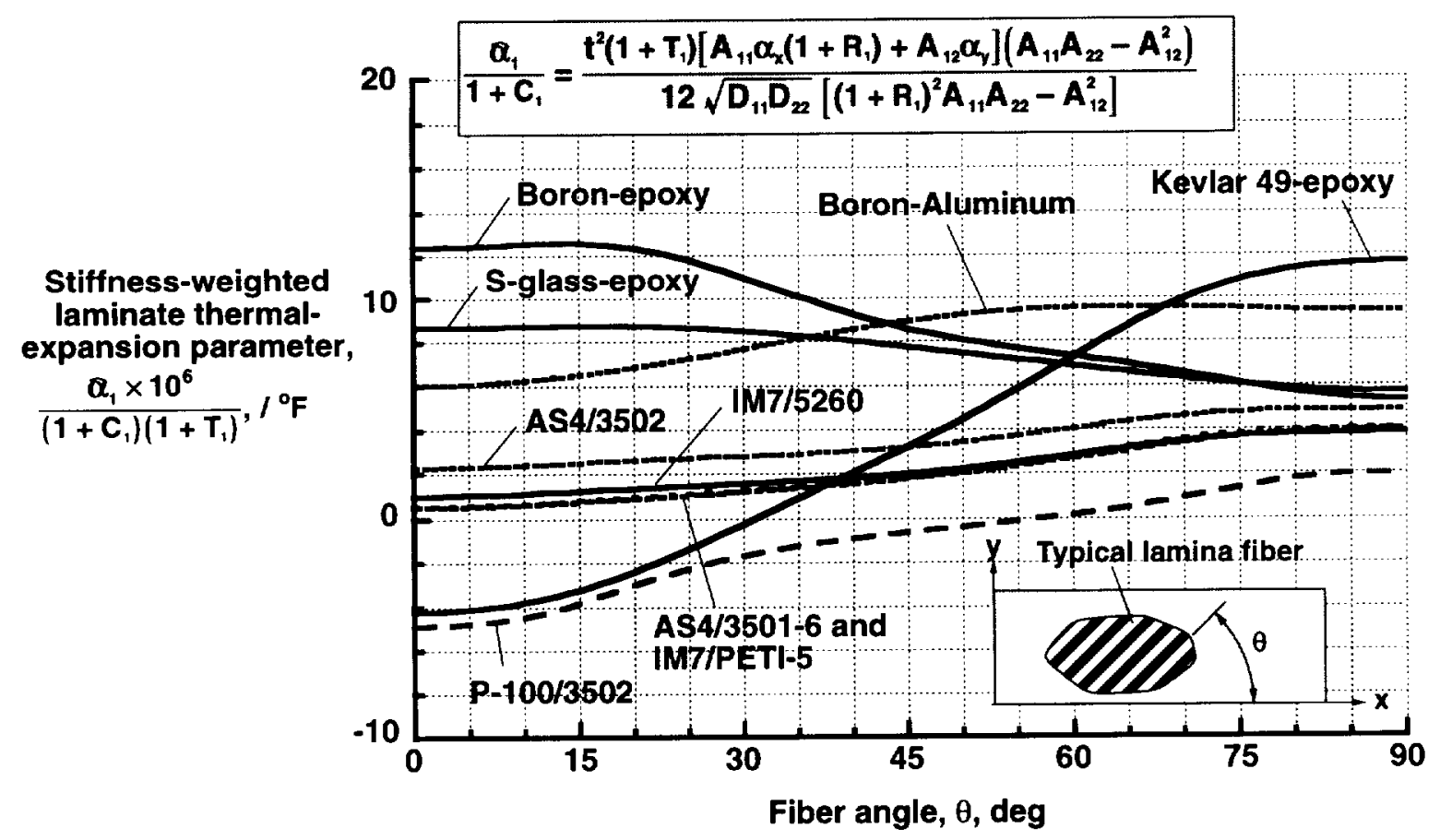

Fig. 30 Effects of lamina material properties on stiffness-weighted laminate thermal-expansion parameter $a_{1} /\left[\left(1+c_{1}\right)\left(1+T_{1}\right)\right]$ for $\left[( \pm \theta)_{m}\right]_{s}$ balanced, angle-ply laminates $\left(R_{2}=R_{1}=0, T_{1}=T_{2}, m=1,2, \ldots\right)$. 


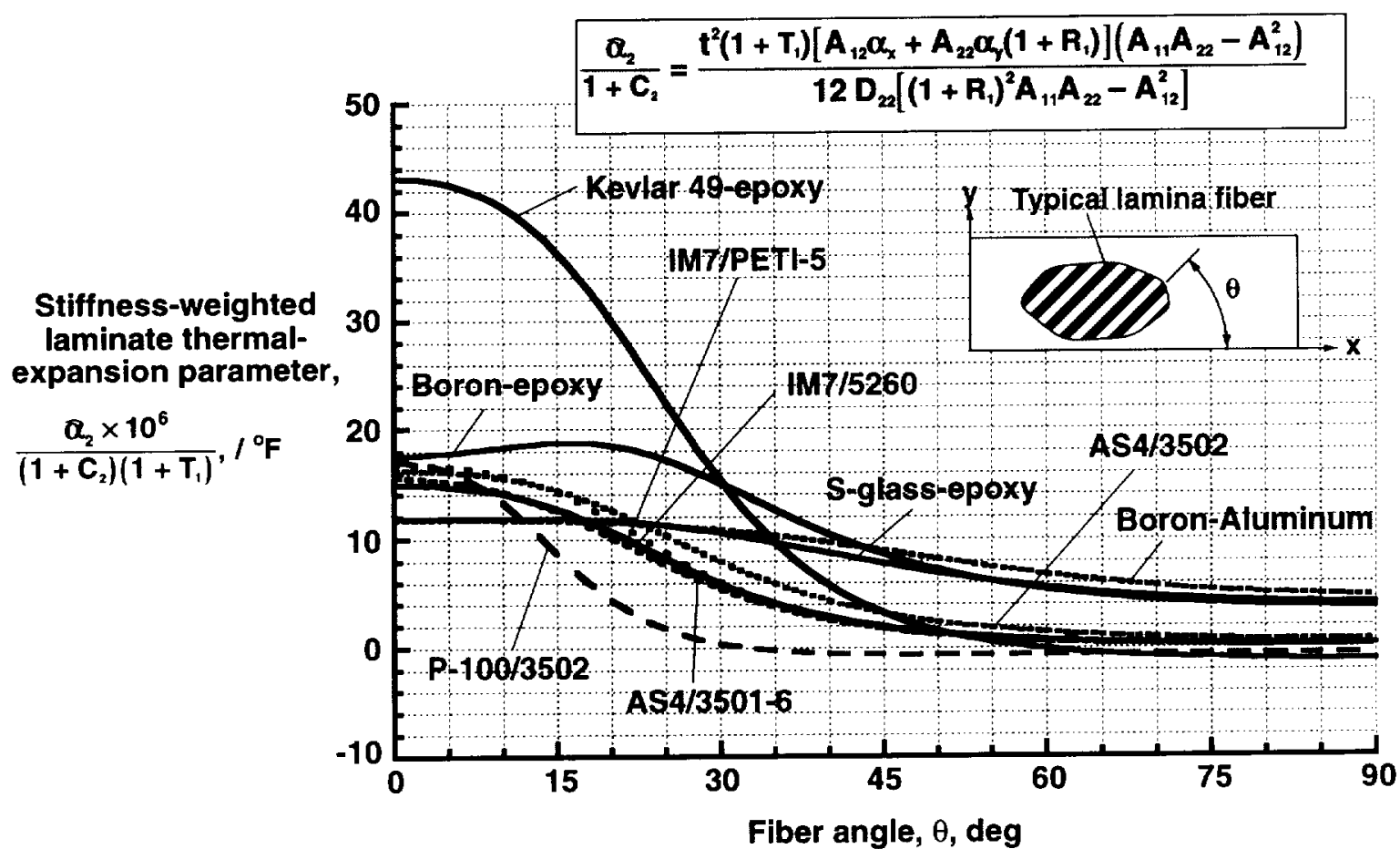

Fig. 31 Effects of lamina material properties on stiffness-weighted laminate thermal-expansion parameter $\alpha_{2}$ $\hat{\alpha}_{2} /\left[\left(1+C_{2}\right)\left(1+T_{1}\right)\right]$ for $\left[( \pm \theta)_{m}\right]_{s}$ balanced, angle-ply laminates $\left(R_{2}=R_{1}=0, T_{1}=T_{2}, m=1,2, \ldots\right)$.

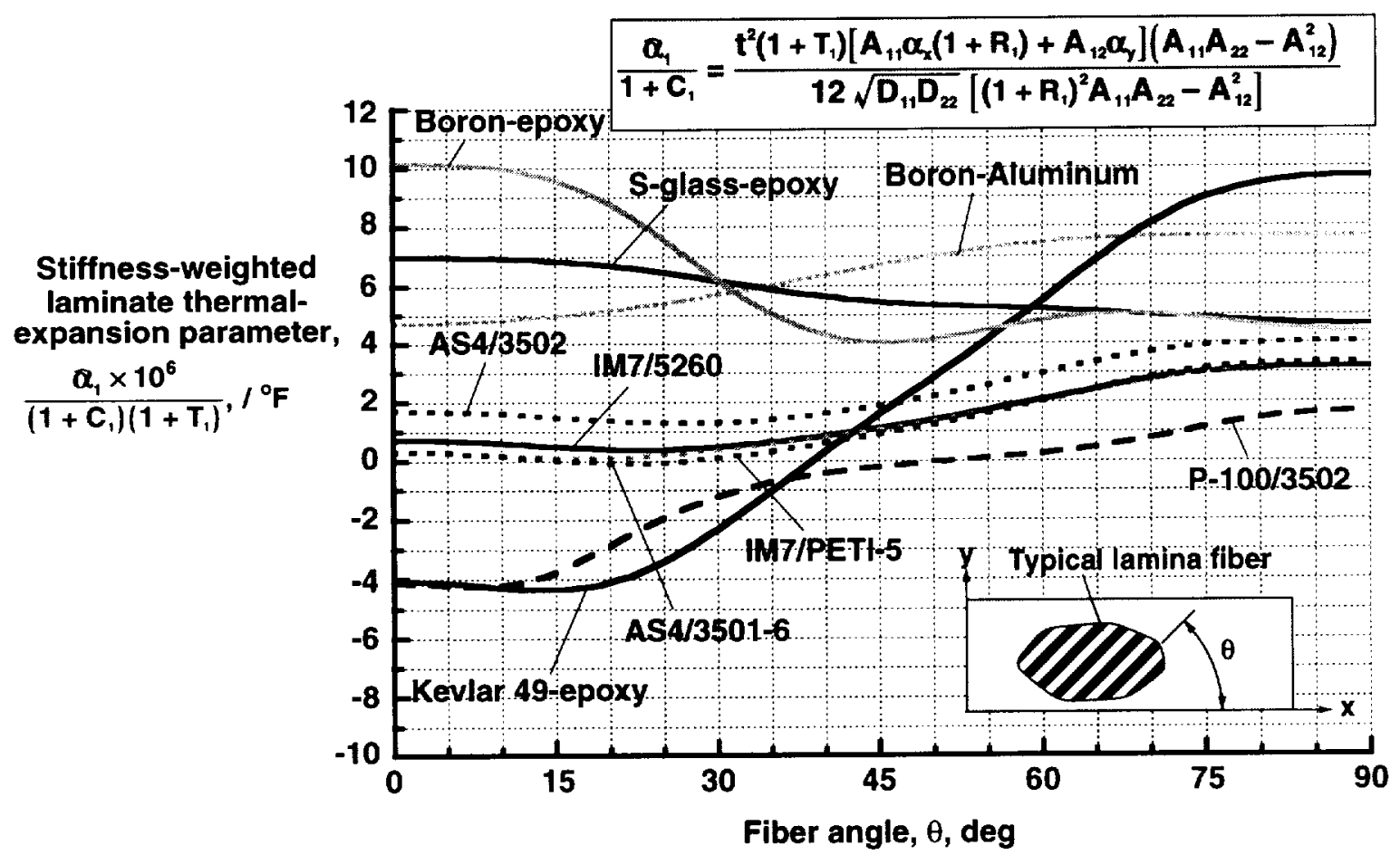

Fig. 32 Effects of lamina material properties on stiffness-weighted laminate thermal-expansion parameter $\alpha_{1} /\left[\left(1+C_{1}\right)\left(1+T_{1}\right)\right]$ for $\left[( \pm \theta)_{m}\right]_{s}$ balanced, angle-ply laminates $\left(R_{2}=R_{1}=0.2, T_{1}=T_{2}, m=1,2, \ldots\right)$. 


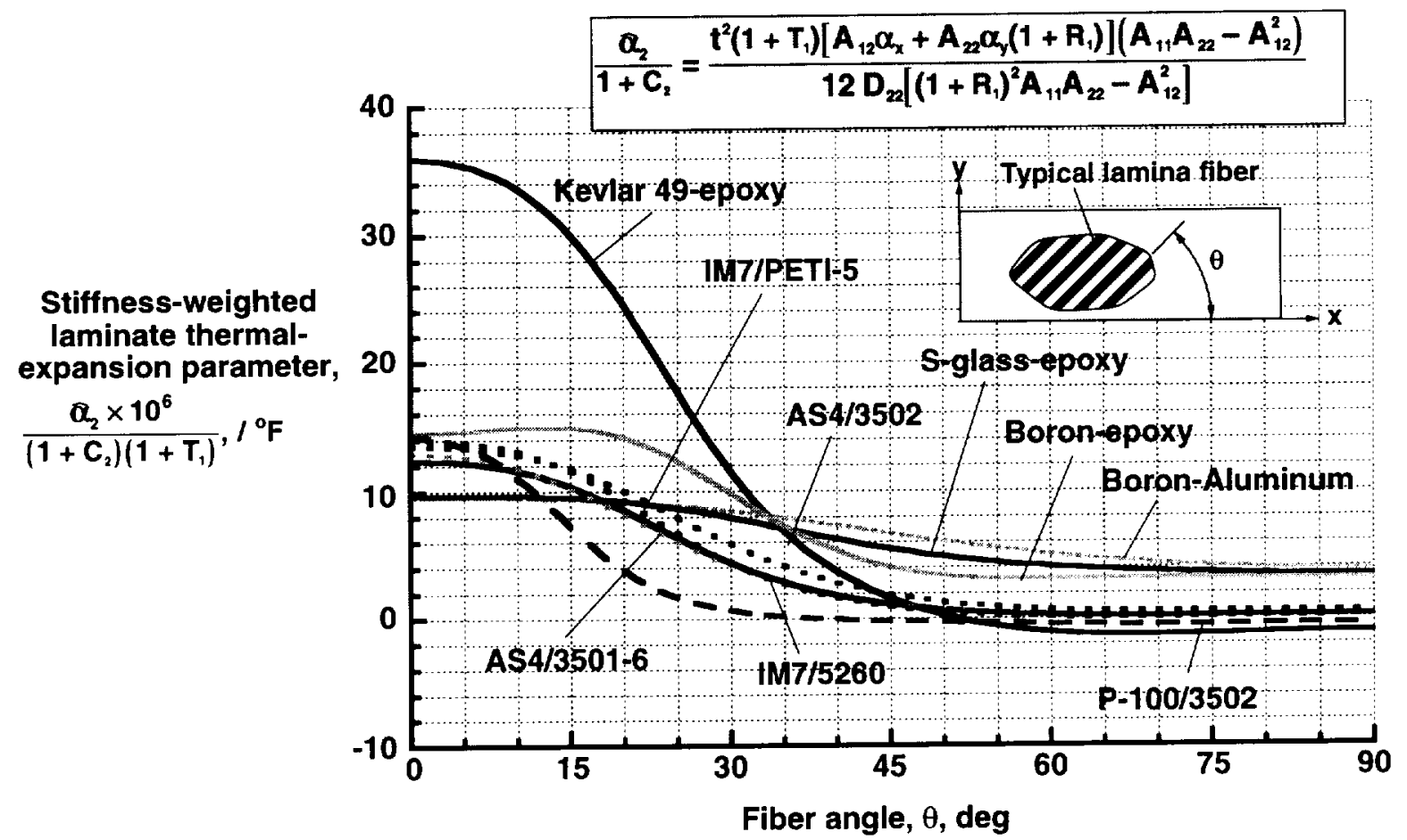

Fig. 33 Effects of lamina material properties on stiffness-weighted laminate thermal-expansion parameter $\alpha_{2} /\left[\left(1+C_{2}\right)\left(1+T_{1}\right)\right]$ for $\left[( \pm \theta)_{m}\right]_{s}$ balanced, angle-ply laminates $\left(R_{2}=R_{1}=0.2, T_{1}=T_{2}, m=1,2, \ldots\right)$.

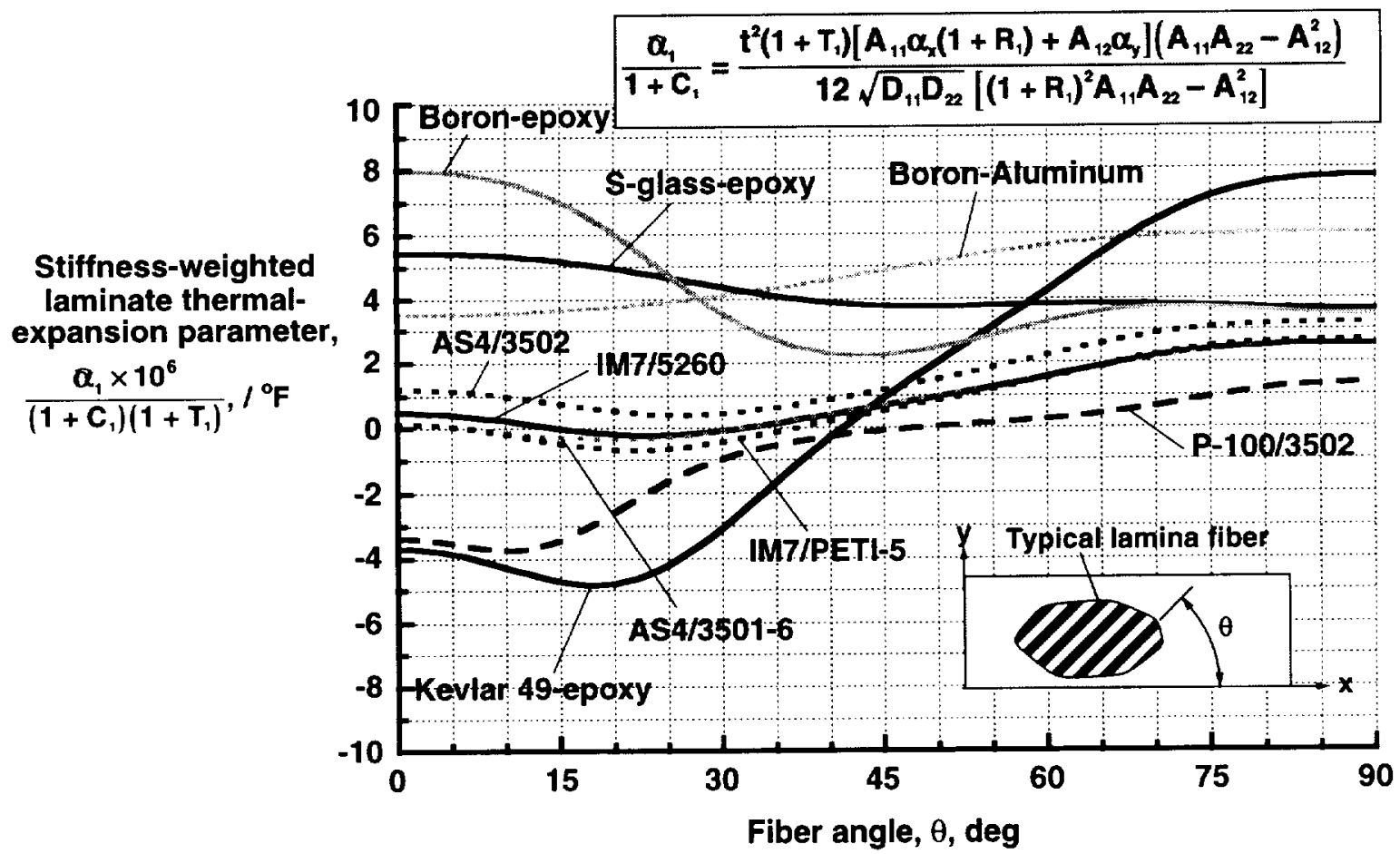

Fig. 34 Effects of lamina material properties on stiffness-weighted laminate thermal-expansion parameter $\alpha_{1} /\left[\left(1+C_{1}\right)\left(1+T_{1}\right)\right]$ for $\left[( \pm \theta)_{m}\right]_{g}$ balanced, angle-ply laminates $\left(R_{2}=R_{1}=0.5, T_{1}=T_{2}, m=1,2, \ldots\right)$. 


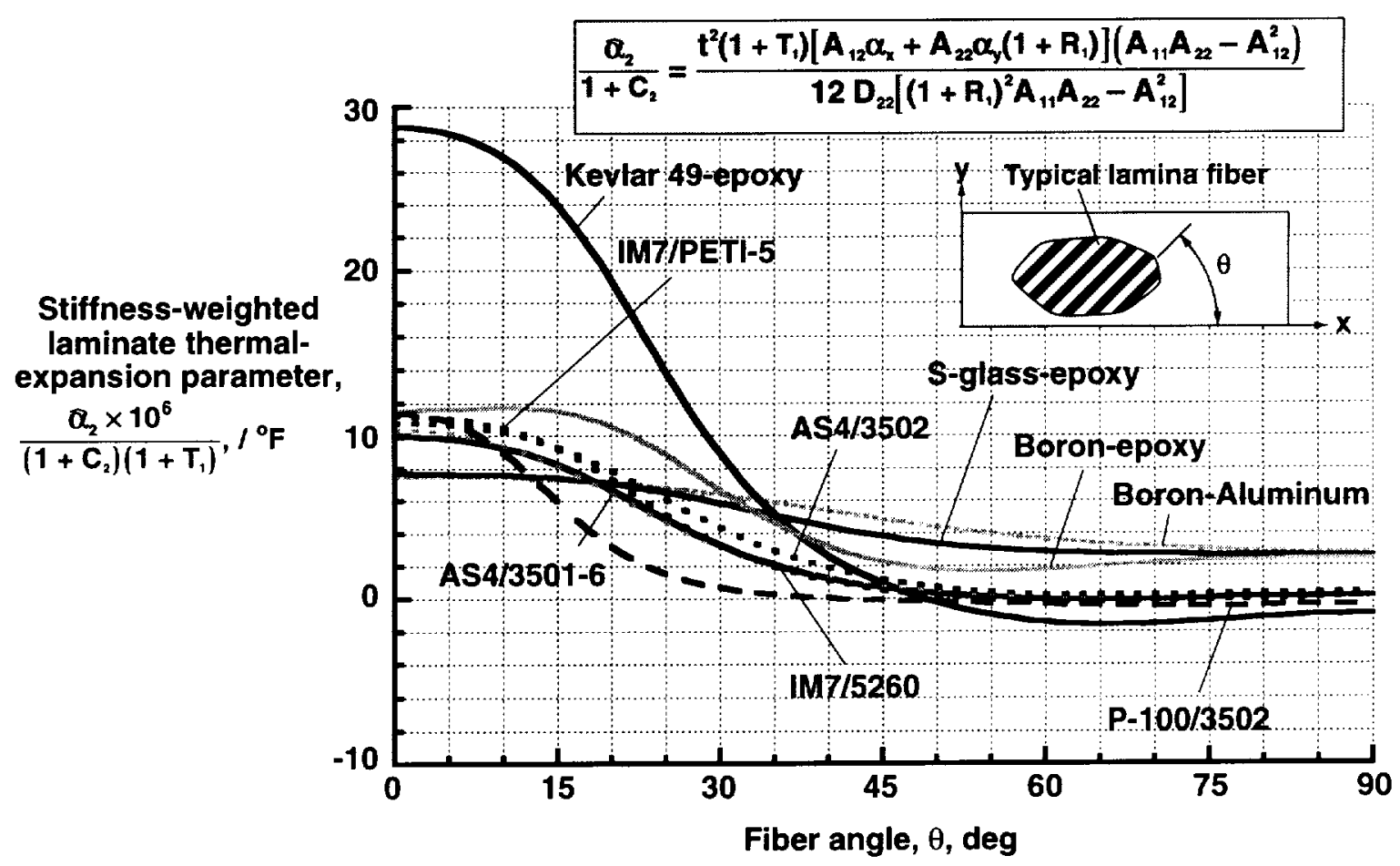

Fig. 35 Effects of lamina material properties on stiffness-weighted laminate thermal-expansion parameter $a_{2} /\left[\left(1+C_{2}\right)\left(1+T_{1}\right)\right]$ for $\left[( \pm \theta)_{m}\right]_{s}$ balanced, angle-ply laminates $\left(R_{2}=R_{1}=0.5, T_{1}=T_{2}, m=1,2, \ldots\right)$. 